TRANSACTIONS OF THE

AMERICAN MATHEMATICAL SOCIETY

Volume 348, Number 12, December 1996, Pages 4691-4736

S 0002-9947(96)01669-8

\title{
ON BIUNITARY PERMUTATION MATRICES AND SOME SUBFACTORS OF INDEX 9
}

\author{
UMA KRISHNAN AND V. S. SUNDER
}

\begin{abstract}
This paper is devoted to a study of the subfactors arising from vertex models constructed out of 'biunitary' matrices which happen to be permutation matrices. After a discussion on the computation of the higher relative commutants of the associated subfactor (in the members of the tower of Jones' basic construction), we discuss the principal graphs of these subfactors for small sizes $(N=k \leq 3)$ of the vertex model. Of the 18 possibly inequivalent such biunitary matrices when $N=3$, we compute the principal graphs completely in 15 cases, all of which turn out to be finite. In the last section, we prove that two of the three remaining cases lead to subfactors of infinite depth and discuss their principal graphs.
\end{abstract}

\section{INTRODUCTION}

The importance of the notion of a commuting square of finite-dimensional $C^{*}$ algebras and its connection with subfactors has been amply demonstrated - for instance, see [GHJ], $[\mathrm{P}]$ and $[\mathrm{P} 2]$. While there is a well-understood prescription for constructing a subfactor of the hyperfinite $I I_{1}$ factor from such a commuting square, what still seems a mystery is the relation between the initial commuting square and the so-called principal (or standard) graph invariant of the subfactor (in the absence of what Ocneanu terms 'flatness of the connection'). In this paper, we examine a seemingly simple class of such commuting squares, namely the ones arising from 'vertex models given by biunitary matrices which also happen to be permutation matrices', and discuss the principal graphs of the associated subfactor. We give below a brief section-by-section description of the contents of this paper.

§2: Notation and Preliminaries: We recall here the definition of the specific family of commuting squares which have been referred to as vertex models, and also the prescription for constructing a subfactor of the hyperfinite $I I_{1}$ factor from a vertex model (or more generally, from a commuting square which is symmetric (in the terminology of [HS]) or non-degenerate (in the terminology of [P2])).

$\S 3$ : Biunitary permutation matrices: Here, we re-cast - in a form that we shall work with in the rest of the paper - what it means to have a vertex model given by a biunitary matrix which also happens to be a permutation matrix; we also define some mappings associated with such matrices, which play a central role in the subsequent analysis.

Received by the editors April 12, 1994.

1991 Mathematics Subject Classification. Primary 46L37.

The first author's research was supported by the National Board for Higher Mathematics in India.

(C)1996 American Mathematical Society 
$\S 4:$ Computation of the higher relative commutants: In this section, we describe a compact prescription (due to Jones) for computing the higher relative commutants (at least in principle), and we identify what this abstract prescription amounts to in our case.

$\S 5$ : Some simple special cases: Here we discuss some particularly simple special cases, and describe the resulting principal graphs, most of which may be described as Cayley graphs for a group or a group-dual with respect to a suitable set of generators.

$\S 6$ : The case $k=N \leq 3$ : We begin by discussing a natural equivalence relation in the set of biunitary permutation matrices. After quickly disposing of the case $N=$ 2 , we go on to show that when $N=3$ there are precisely 18 distinct equivalence classes of such matrices; we explicitly list a matrix from each class, and note that nine of these cases are already covered by the discussion in $\S 5$, and give the principal graph of the associated subfactor.

$\S 7$ : The finite principal graphs (for $N=3$ ) : In this section, we compute the principal graphs of six of the remaining cases, which all turn out to be finite.

§8: Two infinite depth subfactors: In this section, we prove that two of the remaining cases lead to infinite principal graphs, and give a partial description of what those graphs look like.

§9: Concluding Remarks: Here we observe : (a) that all subfactors arising from vertex models given by $9 \times 9$ biunitary permutation matrices are self-dual; (b) the peculiar fact that all the 15 finite principal graphs obtained when $N=3$ turn out to be Cayley graphs of groups or group-duals; and (c) a relation - in the cases computed - between the principal graphs of the subfactors obtained from a biunitary permutation matrix and its adjoint, and raise a natural question.

\section{NOTATION AND PRELIMINARIES}

We shall use the expression 'vertex model', throughout this paper, to mean a commuting square - see $[\mathrm{HS}],[\mathrm{K}],[\mathrm{O}],[\mathrm{OK}],[\mathrm{P}]$ - of the form

$$
\begin{array}{ccc}
u\left(1 \otimes M_{k}(\mathbb{C})\right) u^{*} & \subseteq & M_{N}(\mathbb{C}) \otimes M_{k}(\mathbb{C}) \\
\cup & \cup & \cup \\
\mathbb{C} & \subseteq & M_{N}(\mathbb{C}) \otimes 1
\end{array}
$$

where $u=\left(\left(u_{\beta b}^{\alpha a}\right)\right)$ is a unitary element of $M_{N}(\mathbb{C}) \otimes M_{k}(\mathbb{C})$. (We shall find it convenient to use the convention, at least in $\S \S 2$ - 4, of denoting elements of $\Omega_{N}$ by Greek letters and elements of $\Omega_{k}$ by Roman letters, where we write, here and in the sequel, the symbol $\Omega_{l}$ for the set $\{1,2, \cdots, l\}$. (From $\S 5$ onwards, we consider, for the sake of simplicity, the case $k=N$, and dispense with this Greek vs. Roman convention.) It is well-known that $(\dagger)$ is a commuting square precisely when the unitary matrix $u$ satisfies the following biunitarity condition: if we define the element $\tilde{u}=\left(\left(\tilde{u}_{\beta b}^{\alpha a}\right)\right)$ of $M_{N}(\mathbb{C}) \otimes M_{k}(\mathbb{C})$ by

$$
\tilde{u}_{\beta b}^{\alpha a}=u_{\alpha b}^{\beta a}
$$

then also $\tilde{u}$ is unitary. (Thus $\tilde{u}$ may be thought of as the 'block-transpose' of $u$ in the sense that if we write $u$ in block-form as $u=\left(\left(u_{\beta}^{\alpha}\right)\right)$, then $\tilde{u}_{\beta}^{\alpha}=u_{\alpha}^{\beta}$.)

In the rest of this paper, we shall reserve the term biunitary matrix for a unitary matrix $u \in M_{N}(\mathbb{C}) \otimes M_{k}(\mathbb{C})$ which satisfies the above biunitarity condition. 
If we rewrite the commuting square $(\dagger)$ as

$$
\begin{aligned}
& B_{0} \subseteq B_{1} \\
& \cup \cup \\
& A_{0} \subseteq A_{1}
\end{aligned}
$$

there is a well-known prescription - see [GHJ] - for constructing a subfactor $R_{u}$ of the hyperfinite factor $R$ with $\left[R: R_{u}\right]=k^{2}$. (Namely, let $B_{0} \subset B_{1} \subset B_{2} \subset B_{3} \subset \cdots$ denote the tower obtained by repeated applications of Jones' basic construction - see $[\mathrm{J}],[\mathrm{GHJ}]$; if $e_{n}$ denotes the projection in $B_{n+1}$ which implements the conditional expectation of $B_{n}$ onto $B_{n-1}$, for $n \geq 1$, let $A_{n+1}$ be the subalgebra of $B_{n+1}$ generated by $A_{n} \cup\left\{e_{n}\right\}$; then $R$ and $R_{u}$ are, respectively, the von Neumann algebra completions, with respect to the unique tracial state on these algebras, of $\bigcup B_{n}$ and $\left.\bigcup A_{n} \cdot\right)$

For later reference, we remark - see [O] for the general case, also [KSV] where this special case is explicitly worked out - that there is a natural equivalence relation on the set of biunitary matrices in $M_{N} \otimes M_{k}$, such that the subfactors arising from equivalent biunitary matrices are conjugate. The relation is given by $u_{1} \sim u_{2}$ if and only if there exist unitary matrices $a, c \in M_{N}(\mathbb{C}), b, d \in M_{k}(\mathbb{C})$ such that $u_{1}=(a \otimes b) u_{2}(c \otimes d)$.

\section{Permutation biunitary matrices}

In the rest of this paper, we shall be interested in biunitary matrices which are permutation matrices (as elements of $M_{N k}(\mathbb{C})=M_{N}(\mathbb{C}) \otimes M_{k}(\mathbb{C})$ ). We shall find it convenient to work with an alternative description of such matrices, which we single out in the next lemma.

Lemma 1. Let $u \in M_{N}(\mathbb{C}) \otimes M_{k}(\mathbb{C})$. Then the following conditions on $u$ are equivalent:

(i) $u$ is biunitary, and is further a permutation matrix (i.e., is a 0, 1 matrix);

(ii) there exist permutations $\left\{\rho_{a}: a \in \Omega_{k}\right\} \subset S\left(\Omega_{N}\right),\left\{\lambda_{\alpha}: \alpha \in \Omega_{N}\right\} \subset S\left(\Omega_{k}\right)$ (where we write $S(X)$ for the group of permutations of the set $X$ ) such that:

(a) the equation

$$
\pi(\beta, b)=\left(\rho_{b}(\beta), \lambda_{\beta}(b)\right)
$$

defines a permutation $\pi \in S\left(\Omega_{N} \times \Omega_{k}\right)$; and

(b)

$$
u_{\beta b}^{\alpha a}=\delta_{(\alpha, a), \pi(\beta, b)}=\delta_{\alpha, \rho_{b}(\beta)} \delta_{a, \lambda_{\beta}(b)} .
$$

Proof. $(i) \Rightarrow($ ii $)$; If $u$ is a biunitary 0,1 - valued matrix, then let $\pi \in S\left(\Omega_{N} \times \Omega_{k}\right)$ be defined by $u_{\beta b}^{\alpha a}=\delta_{(\alpha, a), \pi(\beta, b)}$.

Assertion. For any $\beta \in \Omega_{N}, a \in \Omega_{k}$ (resp., $\left.\alpha \in \Omega_{N}, b \in \Omega_{k}\right), \pi\left(\{\beta\} \times \Omega_{k}\right) \cap\left(\Omega_{N} \times\right.$ $\{a\})$ ( resp., $\left.\pi\left(\Omega_{N} \times\{b\}\right) \cap\left(\{\alpha\} \times \Omega_{k}\right)\right)$ is a singleton. Furthermore,

$$
\begin{aligned}
& \pi\left(\{\beta\} \times \Omega_{k}\right) \cap\left(\Omega_{N} \times\{a\}\right)=\left\{\left(\phi_{a}(\beta), a\right)\right\}, \\
& \pi\left(\Omega_{N} \times\{b\}\right) \cap\left(\{\alpha\} \times \Omega_{k}\right)=\left\{\left(\alpha, \psi_{\alpha}(b)\right)\right\}
\end{aligned}
$$

where

$$
\phi_{a}(\beta)=\rho_{\lambda_{\beta}^{-1}(a)}(\beta) \text { and } \psi_{\alpha}(b)=\lambda_{\rho_{b}^{-1}(\alpha)}(b) \text {. }
$$


The first (as well as the parenthetical) statement of the assertion is an immediate consequence of two facts: (i) the hypothesis on $u$ implies that the block-transpose matrix $\tilde{u}$ is also a permutation matrix; and (ii) $\pi(\beta, b)=(\alpha, a) \Leftrightarrow \tilde{u}_{\alpha b}^{\beta a}=1$.

The second assertion follows from the definitions.

The assertion clearly proves the implication $(i) \Rightarrow(i i)$, while the implication $($ ii $) \Rightarrow(i)$ is immediate.

We shall find the following notation convenient.

\section{Definition 2. Define}

$$
\begin{aligned}
P_{N, k}=\{ & \pi \in S\left(\Omega_{N} \times \Omega_{k}\right): \text { there exist } \lambda: \Omega_{N} \rightarrow S\left(\Omega_{k}\right), \rho: \Omega_{k} \rightarrow S\left(\Omega_{N}\right) \\
& \text { such that } \left.\pi(\beta, b)=\left(\rho_{b}(\beta), \lambda_{\beta}(b)\right) \text { for all } \beta \in \Omega_{N}, b \in \Omega_{k}\right\}
\end{aligned}
$$

where we write $\lambda_{\beta}$ (resp., $\rho_{b}$ ) for the image of $\beta$ (resp., $b$ ) under the map $\lambda$ (resp., $\rho$ ). If $\pi, \lambda, \rho$ are related as above, we shall simply write $\pi \leftrightarrow(\rho, \lambda) \in P_{N, k}$. (Later, when we consider the case $N=k$, we shall simply write $P_{N}$ for $P_{N, N}$.)

Thus, Lemma 1 states that there is a bijection between biunitary permutation matrices of size $N k$ and elements $\pi \leftrightarrow(\rho, \lambda) \in P_{N, k}$, given by $u_{\beta b}^{\alpha a}=\delta_{\alpha, \rho_{b}(\beta)} \delta_{a, \lambda_{\beta}(b)}$.

The following proposition, which is an immediate consequence of the definitions, lists some useful properties of the various ingredients of a biunitary permutation.

Proposition 3. Let $\pi \leftrightarrow(\rho, \lambda), \phi_{a}, \psi_{\alpha}$ be as above. Then, for arbitrary $a \in \Omega_{k}, \alpha \in$ $\Omega_{N}$, we have:

(i) $\phi_{a} \in S\left(\Omega_{N}\right), \psi_{\alpha} \in S\left(\Omega_{k}\right)$;

(ii) $\pi^{-1} \leftrightarrow\left(\phi^{-1}, \psi^{-1}\right) \in P_{N, k}$ (i.e., $\left.\pi^{-1}(\alpha, a)=\left(\phi_{a}^{-1}(\alpha), \psi_{\alpha}^{-1}(a)\right)\right)$;

(iii) $\phi_{a}^{-1}(\alpha)=\rho_{\psi_{\alpha}^{-1}(a)}^{-1}(\alpha), \psi_{\alpha}^{-1}(a)=\lambda_{\phi_{a}^{-1}(\alpha)}^{-1}(a)$.

\section{Computation of higher Relative commutants}

Suppose $u \in M_{N}(\mathbb{C}) \otimes M_{k}(\mathbb{C})$ is biunitary; let $R_{u}$ be the subfactor of the hyperfinite factor $R$ constructed as outlined in $\S 2$. Let

$$
R_{-1}=R_{u} \subseteq R_{0}=R \subseteq R_{1} \subseteq R_{2} \subseteq \cdots
$$

be the tower obtained by repeated applications of Jones' basic construction to the inclusion $R_{u} \subseteq R$. Let us write $C_{n}=R_{u}^{\prime} \cap R_{n-1}, n=0,1,2, \cdots$. (Thus, $C_{0}=\mathbb{C}, C_{1}=R_{u}^{\prime} \cap R$, etc.)

We shall find it convenient to work with a diagrammatic description of the tower $\left\{C_{n}: n \geq 0\right\}$ of relative commutants. We pause to briefly recall the conventions for this approach (which is due to Jones ([J1],[BHJ]); also see [JS] for details).

Once and for all, we fix a biunitary matrix $u \in M_{N}(\mathbb{C}) \otimes M_{k}(\mathbb{C})$. When $u$ is a permutation matrix, the symbols $\pi, \rho, \lambda, \phi$ and $\psi$ will have the meanings they had in the last section. 
An element, say $F$, of $\otimes^{n} M_{k}(\mathbb{C})$ will be represented by a 'black box' with two sets of $n$ vertical strands, thus:

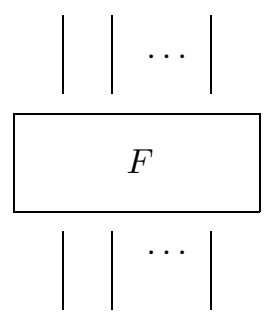

A state (for such a simple diagram) is a labelling of each vertical segment of string with a Roman letter, and the box $F$ is considered as a scalar function on the set of possible states:

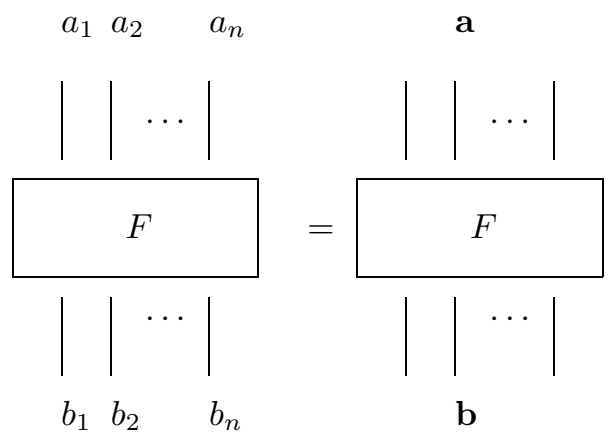

In addition to such boxes, our diagrams will usually contain two kinds of strands - vertical as well as horizontal. We adopt the convention that a state labels segments of horizontal (resp., vertical) strands with Greek (resp., Roman) letters.

Matrix multiplication corresponds to 'concatenation of boxes' in an obvious sense. To be able to stick to this correspondence, we define a state on a (possibly complicated) diagram as a labelling of all 'unbounded' segments of strands according to the convention of the preceding paragraph, and when evaluating the value of a state on a diagram, we sum, over all possible (admissible) labellings of bounded segments, the products of all the 'local contributions' (coming from 'black boxes' as well as from crossings).

Furthermore, all the strands in our diagrams will usually be oriented, so we will encounter two kinds of crossings:

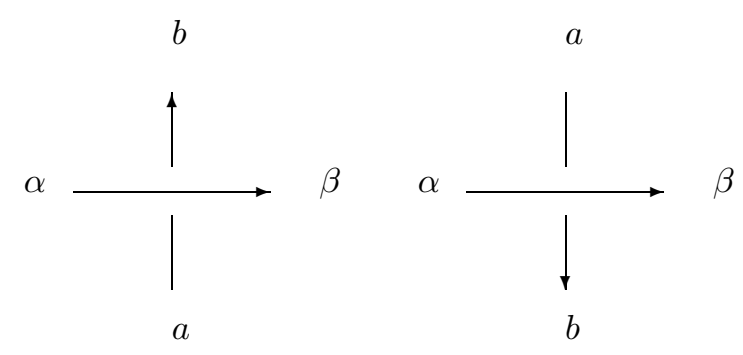

Positive Crossing Negative Crossing 
(All the diagrams that we shall encounter will have the property that at any crossing, the horizontal strand will always be the 'over-string'.)

Given a diagram all segments of all of whose strands have been labelled appropriately, we assign 'Boltzmann weights' to crossings (depending upon the parity of the crossing) by the following prescription:

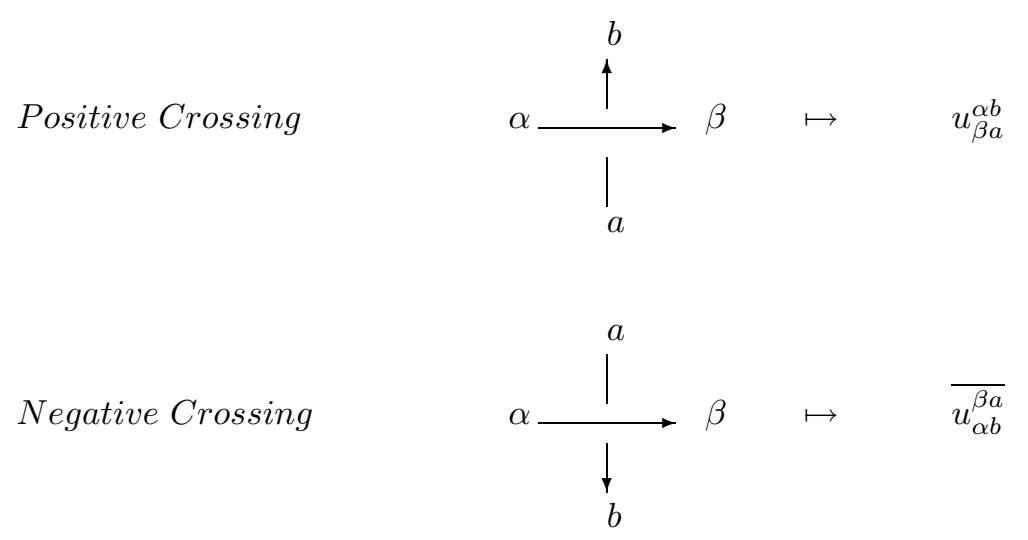

We are finally ready to state Jones' prescription for computing the tower $\left\{C_{n}\right.$ : $n \geq 0\}$ of relative commutants:

Proposition 4 (Jones). If $u \in M_{N}(\mathbb{C}) \otimes M_{k}(\mathbb{C})$ is biunitary, and if $C_{n}$ is (the $(n-1)$-th higher relative commutant) as above, then $C_{n} \subseteq \otimes^{n} M_{k}(\mathbb{C})$; in fact, $C_{n}$ consists of precisely those $F \in \otimes^{n} M_{k}(\mathbb{C})$ for which there exists a $G \in \otimes^{n} M_{k}(\mathbb{C})$ such that the following equation holds:

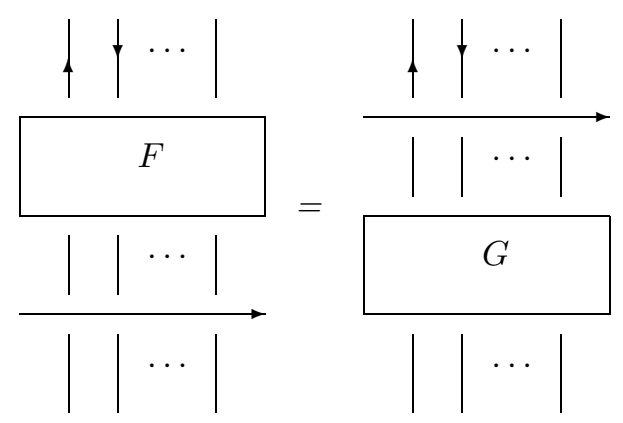

where the vertical strings (on each side of the equality) are alternatively oriented upwards and downwards (starting with the one at the extreme left).

For the rest of this section, we fix a $\pi \leftrightarrow(\rho, \lambda) \in P_{N, k}$ and let $\lambda, \rho, \phi, \psi$ be as in $\S 3$. Thus, if $u$ is the biunitary permutation matrix that corresponds to $(\rho, \lambda)$ as in $\S 3$, then

$$
u_{\beta b}^{\alpha a}=\delta_{\alpha, \rho_{b}(\beta)} \delta_{a, \lambda_{\beta}(b)}
$$

The point of the next lemma is to point out that if $u_{\beta b}^{\alpha a}=1$, then any pair consisting of one Greek letter from $\{\alpha, \beta\}$ and one Roman letter from $\{a, b\}$ determines the complementary pair. We shall find some of these formulae convenient in subsequent computations. 
Lemma 5. If $\alpha, \beta \in \Omega_{N}, a, b \in \Omega_{k}$, then the following conditions are equivalent:

(i) $u_{\beta a}^{\alpha b}=1$

(ii) $\alpha=\rho_{a}(\beta)$ and $b=\lambda_{\beta}(a)$;

(iii) $\beta=\phi_{b}^{-1}(\alpha)$ and $a=\psi_{\alpha}^{-1}(b)$;

(iv) $\beta=\rho_{a}^{-1}(\alpha)$ and $b=\psi_{\alpha}(a)$;

(v) $\alpha=\phi_{b}(\beta)$ and $a=\lambda_{\beta}^{-1}(b)$.

Proof. $(i) \Leftrightarrow(i i)$ by definition.

(ii) $\Leftrightarrow$ (iii) by Proposition 3 (ii).

(ii) $\Leftrightarrow(i v)$ by the formula for $\phi^{-1}$ given in Proposition 3 (iii).

(iii) $\Leftrightarrow(v)$ by the formula for $\psi^{-1}$ given in Proposition 3 (iii).

It follows immediately from the preceding lemma that the Boltzmann weights associated with the two kinds of crossings (when we work with a biunitary permutation matrix) are as follows:

Positive Crossing

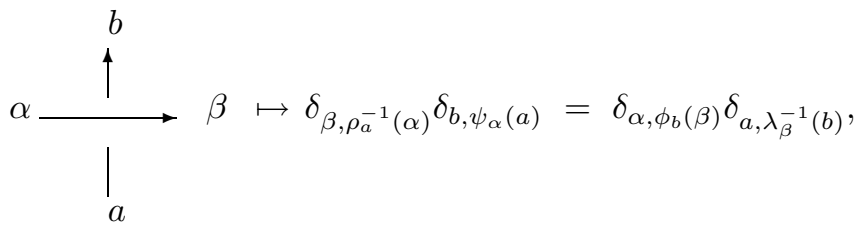

Negative Crossing $\quad \alpha \underset{\downarrow_{b}}{\stackrel{\left.\right|^{a}}{\longrightarrow}} \beta \mapsto \delta_{\beta, \rho_{b}(\alpha)} \delta_{a, \lambda_{\alpha}(b)}=\delta_{\alpha, \phi_{a}^{-1}(\beta)} \delta_{b, \psi_{\beta}^{-1}(a)}$.

Notation. Given a biunitary permutation $u$ and corresponding maps $\lambda, \rho, \phi, \psi$ as above, then for arbitrary $n \geq 1$ and $\mathbf{a} \in \Omega_{k}^{n}$, we define the alternating products

$$
\rho_{\mathbf{a}}=\rho_{a_{1}} \rho_{a_{2}}^{-1} \rho_{a_{3}} \cdots \rho_{a_{n}}^{ \pm}
$$

and

$$
\phi_{\mathbf{a}}=\phi_{a_{1}} \phi_{a_{2}}^{-1} \phi_{a_{3}} \cdots \phi_{a_{n}}^{ \pm}
$$

We are now ready to introduce certain mappings that will play a central role in the computation of the higher relative commutants.

Proposition 6. (i) For all $n \geq 1$, there exists a mapping $\Omega_{N} \ni \alpha \mapsto L_{\alpha}^{(n)} \in$ $S\left(\Omega_{k}^{n}\right)$ such that 
$\alpha$

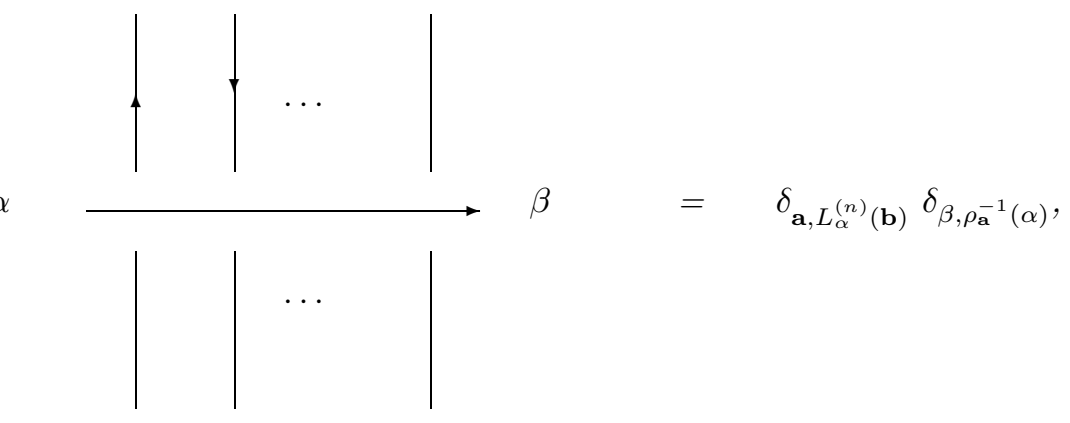

a

where the $L_{\alpha}^{(n)}$ 's are defined as in (ii) below.

(ii) $L_{\alpha}^{(1)}=\psi_{\alpha}^{-1}$; and if $n>1$ and if $L_{\alpha}^{(n)}(\mathbf{b})=\mathbf{a}$, then

$$
\mathbf{a}_{n-1]}=L_{\alpha}^{(n-1)}\left(\mathbf{b}_{n-1]}\right)
$$

(where we have used the obvious notation $\mathbf{a}_{n-1}$ to mean $\left(a_{1}, \cdots, a_{n-1}\right)$ if $\mathbf{a}=$ $\left.\left(a_{1}, \cdots, a_{n}\right)\right) ;$ and

$$
a_{n}= \begin{cases}\lambda_{\phi_{\mathbf{b}_{n-1]}}^{-1}(\alpha)}^{-1}\left(b_{n}\right) & \text { if } n \text { is even }, \\ \psi_{\phi_{\mathbf{b}_{n-1]}^{-1}}^{-1}(\alpha)}^{-1}\left(b_{n}\right) & \text { if } n \text { is odd } .\end{cases}
$$

(iii) $\rho_{L_{\alpha}^{(n)}(\mathbf{b})}^{-1}(\alpha)=\phi_{\mathbf{b}}^{-1}(\alpha)$.

Proof. The proof is a direct consequence of the prescription, given in equation (4), for the Boltzmann weights associated to positive and negative crossings. (For (iii), the two prescriptions given for each kind of crossing must be used in conjunction.)

Remark 7. If it so happens that $\lambda_{\alpha}=\psi_{\alpha}, \rho_{a}=\phi_{a}$ for all $\alpha, a$ (which is actually the case more often than one might expect - see $\S 6$ ), then it is seen from Proposition 6 (ii) that

$$
L_{\alpha}^{(n+1)}(\mathbf{a}, a)=\left(L_{\alpha}^{(n)}(\mathbf{a}), \lambda_{\rho_{\mathbf{a}}^{-1}(\alpha)}^{-1}(a)\right)
$$

for all $\mathbf{a} \in \Omega_{k}^{n}, a \in \Omega_{k}, \alpha \in \Omega_{N}, n=1,2, \cdots$.

In the following, we fix a biunitary $u$, with associated $\lambda, \rho, \phi, \psi$ as above, and let $\left\{C_{n}\right\}$ denote the sequence of higher relative commutants for this $R_{u}$.

Lemma 8. With the identification $\otimes^{n} M_{k}(\mathbb{C})=\operatorname{Mat}_{\Omega_{k}^{n}}(\mathbb{C})$, we have:

$$
\begin{array}{r}
C_{n}=\left\{F=\left(\left(F_{\mathbf{b}}^{\mathbf{a}}\right)\right) \in \operatorname{Mat}_{\Omega_{k}^{n}}(\mathbb{C}): F_{\mathbf{b}}^{\mathbf{a}}=\delta_{\rho_{L_{\alpha}^{(n)}(\mathbf{a})}, \rho_{L_{\alpha}^{(n)}(\mathbf{b})} F_{L_{\beta}^{(n)}-1}^{L_{\alpha}^{(n)}-1} L_{\alpha}^{(n)}(\mathbf{a})}^{\left(\mathbf{b}^{(n)}\right)}\right. \\
\text { for all } \left.\alpha, \beta \in \Omega_{N}, \mathbf{a}, \mathbf{b} \in \Omega_{k}^{n}\right\} .
\end{array}
$$


Proof. In the notation of Proposition 4, we see, from Proposition 6, that on the one hand,

b

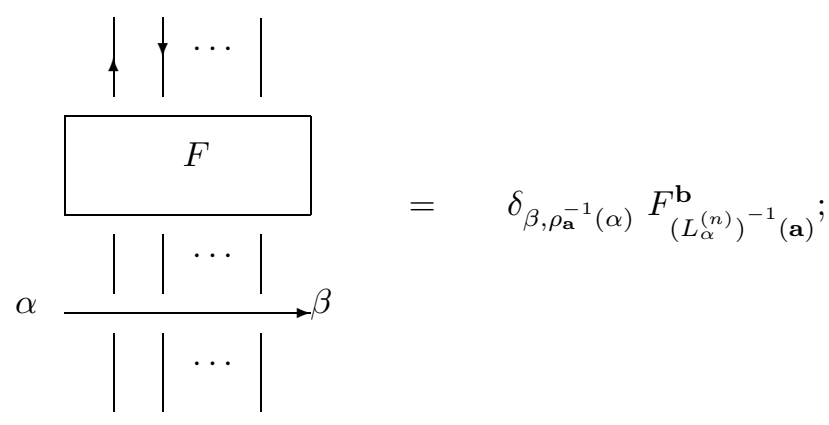

a

on the other hand, we also find that

b

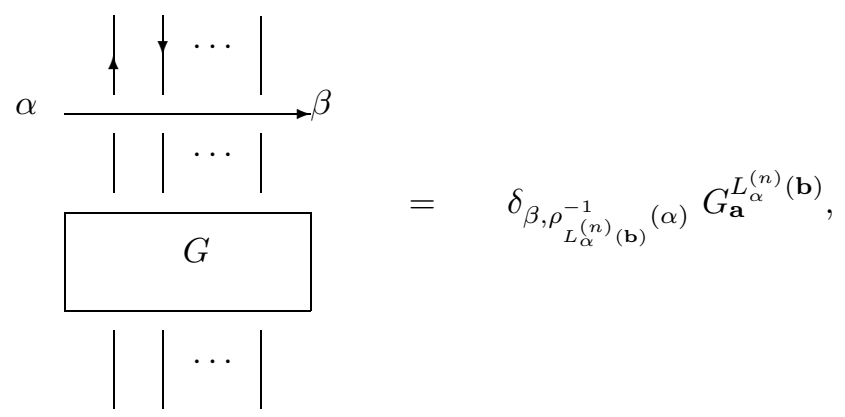

a

which, in view of Proposition 6(iii), is seen to be equal to $\delta_{\beta, \phi_{\mathbf{b}}^{-1}(\alpha)} G_{\mathbf{a}}^{L_{\alpha}^{(n)}(\mathbf{b})}$.

Thus, we find that $C_{n}$ consists of those $F \in \otimes^{n} M_{k}(\mathbb{C})$ for which there exists a $G \in \otimes^{n} M_{k}(\mathbb{C})$ such that

$$
\delta_{\beta, \rho_{\mathbf{a}}^{-1}(\alpha)} F_{\left(L_{\alpha}^{(n)}\right)^{-1}(\mathbf{a})}^{\mathbf{b}}=\delta_{\beta, \phi_{\mathbf{b}}^{-1}(\alpha)} G_{\mathbf{a}}^{L^{(n)}(\mathbf{b})} .
$$

Using the substitution $\mathbf{c}=\left(L_{\alpha}^{(n)}\right)^{-1}(\mathbf{a})$, the last equation may be rewritten again using Proposition 6(iii) - in a more symmetric form as

$$
\delta_{\beta, \phi_{\mathbf{c}}^{-1}(\alpha)} F_{\mathbf{c}}^{\mathbf{b}}=\delta_{\beta, \phi_{\mathbf{b}}^{-1}(\alpha)} G_{L_{\alpha}^{(n)}(\mathbf{c})}^{L_{\alpha}^{(n)}(\mathbf{b})} .
$$

This is easily seen to imply that

$$
F_{\mathbf{c}}^{\mathbf{b}}=\delta_{\phi_{\mathbf{b}}^{-1}, \phi_{\mathbf{c}}^{-1}} G_{L_{\alpha}^{(n)}(\mathbf{c})}^{L^{(n)}(\mathbf{b})}
$$

for arbitrary $\alpha \in \Omega_{N}, \mathbf{b}, \mathbf{c} \in \Omega_{k}^{n}$, and also (as a result of Proposition 6(iii)) that

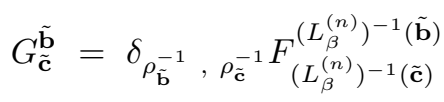


for arbitrary $\beta \in \Omega_{N}, \tilde{\mathbf{b}}, \tilde{\mathbf{c}} \in \Omega_{k}^{n}$. The proof of the lemma is completed by putting together equations (7) and (8) and using the fact, which is a consequence of Proposition 6(iii), that

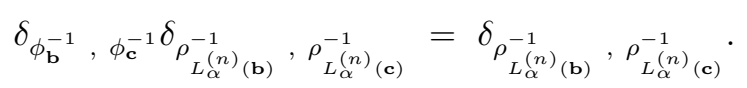

The next lemma is the final ingredient necessary for the identification, in an abstract sense, of the higher relative commutants.

Lemma 9. Let $\Omega$ be a finite set. Suppose we are given an equivalence relation $\sim_{0}$ on $\Omega$ and a subset $\mathcal{L} \subseteq S(\Omega)$ such that $\mathcal{L}=\mathcal{L}^{-1}=\left\{\sigma^{-1}: \sigma \in \mathcal{L}\right\}$. Let $A=\left\{x=\left(\left(x_{j}^{i}\right)\right) \in \operatorname{Mat}_{\Omega}(\mathbb{C}): x_{j}^{i}=\delta_{[i]_{0},[j]_{0}} x_{\sigma(j)}^{\sigma(i)}\right.$ for all $\left.i, j \in \Omega, \sigma \in \mathcal{L}\right\}$, where $[i]_{0}=\left\{j \in \Omega: i \sim_{0} j\right\}$.

Define the equivalence relation $\sim$ on $\Omega$ by requiring that $i \sim j \Leftrightarrow \sigma(i) \sim_{0}$ $\sigma(j)$ for all $\sigma \in G$, where $G$ is the subgroup of $S(\Omega)$ generated by $\mathcal{L}$. Then $G$ acts on the set of $\sim$-equivalence classes ( by $\sigma \cdot[i]=[\sigma(i)]$, where of course $[i]=\{j \in \Omega: i \sim j\})$. Suppose the set of $\sim$-equivalence classes breaks up as a disjoint union of $l$ orbits under this action of $G$.

For $1 \leq p \leq l$, fix one equivalence class $\left[i_{p}\right]$ from the $p$-th orbit, let $H_{p}=\{\sigma \in$ $\left.G: \sigma \cdot\left[i_{p}\right]=\left[i_{p}\right]\right\}$ be the isotropy group of that equivalence class, and let $\pi_{p}$ denote the natural permutation representation of $H_{p}$ on $\left[i_{p}\right]$. Then,

$$
A \cong \bigoplus_{p=1}^{l} \pi_{p}\left(H_{p}\right)^{\prime} .
$$

Proof. To begin with, if $\sigma_{1}, \sigma_{2} \in \mathcal{L}$, note that, for any $x$ in $A$, we have

$$
x_{j}^{i}=\delta_{[i]_{0},[j]_{0}} x_{\sigma_{2}(j)}^{\sigma_{2}(i)}=\delta_{[i]_{0},[j]_{0}} \delta_{\left[\sigma_{2}(i)\right]_{0},\left[\sigma_{2}(j)\right]_{0}} x_{\sigma_{1} \sigma_{2}(j)}^{\sigma_{1} \sigma_{2}(i)}
$$

Since $\mathcal{L}=\mathcal{L}^{-1}$, clearly $G=\left\{\sigma_{1} \sigma_{2} \cdots \sigma_{r}: r \geq 0, \sigma_{1}, \cdots, \sigma_{r} \in \mathcal{L}\right\}$, and it easily follows now that

$$
A=\left\{x=\left(\left(x_{j}^{i}\right)\right) \in \operatorname{Mat}_{\Omega}(\mathbb{C}): x_{j}^{i}=\delta_{[i],[j]} x_{\sigma(j)}^{\sigma(i)} \forall i, j \in \Omega, \sigma \in G\right\} .
$$

Suppose now that $\left\{\left[j_{1}^{(p)}\right], \cdots\left[j_{t_{p}}^{(p)}\right]\right\}$ is the $p$-th orbit in the set of $\sim$-equivalence classes under the $G$-action, and suppose $j_{1}^{(p)}=i_{p}$. For $1 \leq s \leq t_{p}$, fix $\sigma_{s}^{(p)} \in G$ such that $\sigma_{s}^{(p)} \cdot\left[i_{p}\right]=\left[j_{s}^{(p)}\right]$. Assume that the elements of $\Omega$ have been so ordered that $\sigma_{s}^{(p)}$, as a map of $\left[j_{1}^{(p)}\right]$ onto $\left[j_{s}^{(p)}\right]$, is order-preserving. It is then fairly easy to see that $x \in A$ if and only if $x$ has the block-diagonal form

$$
x=\bigoplus_{p=1}^{l} \bigoplus_{s=1}^{t_{p}} x_{s}^{(p)}
$$

with respect to the decomposition

$$
\Omega=\coprod_{p=1}^{l} \coprod_{s=1}^{t_{p}}\left[j_{s}^{(p)}\right],
$$

where $x_{1}^{(p)}=\cdots=x_{t_{p}}^{(p)} \in \pi_{p}\left(H_{p}\right)^{\prime}$.

Putting the previous two lemmas together by considering the specialisation of Lemma 9 to the case where $\Omega=\Omega_{k}^{n}, \mathbf{a} \sim_{0} \mathbf{b} \Leftrightarrow \rho_{L_{\alpha}^{(n)}(\mathbf{a})}^{-1}=\rho_{L_{\alpha}^{(n)}(\mathbf{b})}^{-1} \forall \alpha \in$ 
$\Omega_{N}$, and $\mathcal{L}=\left\{\left(L_{\beta}^{(n)}\right)^{-1}\left(L_{\alpha}^{(n)}\right): \alpha, \beta \in \Omega_{N}\right\}$, we can summarize the contents of this section as follows:

Proposition 10. Let $u \in M_{N}(\mathbb{C}) \otimes M_{k}(\mathbb{C})$ be a biunitary permutation matrix and let $\lambda, \rho, \phi, \psi$ have their usual meaning. Let $R_{u} \subseteq R$ be the hyperfinite (subfactor, factor)- pair corresponding to $u$, and let $C_{n}=R_{u}^{\prime} \cap R_{n-1}, n \geq 0$, where $R_{u}=R_{-1} \subseteq R=R_{0} \subseteq R_{1} \subseteq R_{2} \subseteq \cdots$ is the tower of the basic construction. Then, for $n=1,2, \cdots$, the algebra $C_{n}$ has the following description:

Let $L_{\alpha}^{(n)}$ be defined as in Proposition 6; let $G_{n}$ be the subgroup of $S\left(\Omega_{k}^{n}\right)$ generated by $\left\{L_{\beta}^{(n)^{-1}} L_{\alpha}^{(n)}: \alpha, \beta \in \Omega_{N}\right\}$; and let $\sim_{n}$ be the equivalence relation defined on $\Omega_{k}^{n}$ by

$$
\mathbf{a} \sim_{n} \mathbf{b} \Leftrightarrow \rho_{L_{\alpha}^{(n)}(\sigma(\mathbf{a}))}=\rho_{L_{\alpha}^{(n)}(\sigma(\mathbf{b}))} \forall \sigma \in G_{n}, \alpha \in \Omega_{N} .
$$

Suppose the set of equivalence classes in $\Omega_{k}^{n}$ breaks up into $l_{n}$ orbits under the $G_{n}$ action; fix an equivalence class $\left[\alpha_{p}\right]$ in the $p$-th orbit of equivalence classes, and let $H_{p}=\left\{\sigma \in G_{n}: \sigma\left(\alpha_{p}\right) \sim_{n} \alpha_{p}\right\}$. If $\pi_{p}$ is the natural permutation representation of $H_{p}$ on $\left[\alpha_{p}\right]$, then

$$
C_{n} \simeq \bigoplus_{p=1}^{l_{n}} \pi_{p}\left(H_{p}\right)^{\prime} .
$$

\section{Some Simple SPECIAL CASES}

From now on, we assume, for the sake of simplicity, that $N=k$, although it should be clear how every statement can be naturally modified in the general case. In particular, we shall drop the convention in the preceding sections concerning Greek and Roman letters. Instead, in the interest of typographical convenience, we shall typically use Greek letters for elements of $\Omega_{N}^{n}, n>1$, and reserve Roman letters to denote elements of $\Omega_{N}$. Also, we shall freeze the symbol $N$ to denote what was so far denoted by $N$ or $k$, and we shall use the symbol $k$ as a 'free variable' ranging over the positive integers.

In this section, we consider four special cases, in which the subfactor is necessarily of finite depth, and the prinicipal graph describing the tower $\left\{R_{u}^{\prime} \cap R_{k}: k \geq-1\right\}$ admits a complete and satisfactory description.

Case (0) : $\lambda \equiv i d, \rho \equiv i d$.

In this most trivial example, $\lambda_{i}=\rho_{j}=i d_{\Omega_{n}}$ for all $i, j \in \Omega_{N}$. Then $\pi(i, j)$ $=(i, j)$, and the associated subfactor $R_{u}$ of $R=\bigotimes_{k=1}^{\infty} M_{N}(\mathbb{C})$ may be identified with $1 \otimes \bigotimes_{k=2}^{\infty} M_{N}(\mathbb{C})$, and the principal graph consists of two vertices with $N$ bonds between them.

Case (1) : $\lambda \equiv i d, \rho$ arbitrary.

Let $\rho: \Omega_{N} \rightarrow S\left(\Omega_{N}\right)$ be an arbitrary map, and let $\lambda_{i}=i d_{\Omega_{N}}$ for all $i$ in $\Omega_{N}$. Thus $\pi(i, j)=\left(\rho_{j}(i), j\right)$, which clearly defines a permutation of $\Omega_{N} \times \Omega_{N}$; i.e., $\pi \leftrightarrow(\rho, \lambda) \in P_{N}$. Then observe that

$$
\phi_{i}(j)=\rho_{\lambda_{j}^{-1}(i)}(j)=\rho_{i}(j), \quad \psi_{i}(j)=\lambda_{\rho_{j}^{-1}(i)}(j)=j=\lambda_{i}(j)
$$

and thus $\phi=\rho, \psi=\lambda$. Hence, by Remark 7, we see that, for all $k \geq 1$,

$$
L_{x}^{(k+1)}(\alpha, a)=\left(L_{x}^{(k)}(\alpha), \lambda_{\rho_{\alpha}^{-1}(x)}^{-1}(a)\right)=\left(L_{x}^{(k)}(\alpha), a\right),
$$


for all $x, a \in \Omega_{N}, \alpha \in \Omega_{N}^{k}, k \geq 1$; hence, inductively, we find that $L_{x}^{(k)}=i d_{\Omega_{N}^{k}}$ for all $k \geq 1$ and for all $x \in \Omega_{N}$. In this case, the equivalence classes of $\Omega_{N}^{k}$ are the sets $E_{\sigma}=\left\{\alpha \in \Omega_{N}^{k}: \rho_{\alpha}=\sigma\right\}$, as $\sigma$ ranges over the group $G_{0}$ generated by $\left\{\rho_{i}: i \in \Omega_{N}\right\}$

In fact, it follows from Theorem 11 of [KSV] that in this case, the principal graph $\mathcal{G}$ (describing the tower $\left\{C_{k}: k \geq 0\right\}$ of relative commutants) has the following description: let $\tilde{\mathcal{G}}$ be the bipartite graph with the sets $\tilde{\mathcal{G}}^{(0)}$ and $\tilde{\mathcal{G}}^{(1)}$ of even and odd vertices given by $\tilde{\mathcal{G}}^{(j)}=G_{0} \times\{j\}$, and suppose $\left(g_{0}, 0\right)$ is connected to $\left(g_{1}, 1\right)$ by $\Lambda\left(g_{0}, g_{1}\right)$ bonds, where $\Lambda\left(g_{0}, g_{1}\right)=\#\left\{i \in \Omega_{N}: g_{1}=\omega g_{0} \rho_{i}, \omega \in \mathbb{C},|\omega|=1\right\}$; then $\mathcal{G}$ is the connected component in $\tilde{\mathcal{G}}$ containing the vertex $(1,0)$, where 1 denotes the identity element of $G_{0}$.

It should be remarked that these are precisely the 'diagonal subfactors' of Popa (see [P2]) for appropriate finite groups.

Case (2) : $\rho \equiv i d, \lambda$ arbitrary.

Let $\lambda: \Omega_{n} \rightarrow S\left(\Omega_{N}\right)$ be an arbitrary map and let $\rho_{j}=i d_{\Omega_{N}}$ for all $j$; thus, $\pi(i, j)=\left(i, \lambda_{i}(j)\right)$, which is again clearly a permutation of $\Omega_{N}$, whence $\pi \leftrightarrow(\rho, \lambda) \in P_{N}$. We assume, for simplicity, that $\lambda_{1}=i d$. Observe again that $\phi_{i}(j)=\rho_{\lambda_{j}^{-1}(i)}(j)=j=\rho_{i}(j)$ and that $\psi_{i}(j)=\lambda_{\rho_{j}^{-1}(i)}(j)=\lambda_{i}(j)$, so that $\phi=\rho, \psi=\lambda$. Again, by Remark 7, we have

$$
L_{x}^{(k+1)}(\alpha, a)=\left(L_{x}^{(k)}(\alpha), \lambda_{\rho_{\alpha}^{-1}(x)}^{-1}(a)\right)=\left(L_{x}^{(k)}(\alpha), \lambda_{x}^{-1}(a)\right)
$$

and hence, inductively, we see that

$$
L_{x}^{(k)}=\lambda_{x}^{-1} \times \lambda_{x}^{-1} \times \cdots \times \lambda_{x}^{-1} .
$$

If $G_{1}$ denotes the subgroup of $S\left(\Omega_{N}\right)$ generated by $\left\{\lambda_{i}: i \in \Omega_{N}\right\}$, we see that, in the notation of Proposition 10, $G_{k}=\left\{\sigma \times \sigma \times \cdots \times \sigma: \sigma \in G_{1}\right\}$, that the equivalence relation on $\Omega_{N}^{k}$ is the trivial one $(\alpha \sim \beta$ for all $\alpha, \beta)$ - as a result of the triviality of the $\rho_{i}$ 's - and if $\pi$ denotes the natural representation of $G_{1}$ on $\mathbb{C}^{N}$, then $C_{k} \cong(\pi \otimes \pi \otimes \cdots \otimes \pi)\left(G_{1}\right)^{\prime}$.

Here, too, it follows from Theorem 10 of [KSV] that the principal graph $\mathcal{G}$ has the following description: let $\tilde{\mathcal{G}}$ be the bipartite graph with the set of even (resp., odd) vertices being given by $\tilde{\mathcal{G}}^{(0)}=\hat{G}_{1} \times\{0\}$ (resp., $\tilde{\mathcal{G}}^{(1)}=\hat{G}_{1} \times\{1\}$ ), where $\hat{G}$ denotes the collection of equivalence classes of irreducible representations of $G$, and with the number of bonds joining $\left(\pi_{0}, 0\right)$ and $\left(\pi_{1}, 1\right)$ given by $\Lambda\left(\pi_{0}, \pi_{1}\right)=\left\langle\pi_{0} \otimes \pi, \pi_{1}\right\rangle$ where $\pi$ is the given representation of $G_{1}$ on $\mathbb{C}^{N}$. Then $\mathcal{G}$ is the connected component of $\tilde{\mathcal{G}}$ containing $(1,0)$, where 1 denotes the trivial representation of $G_{1}$.

Case (3) : This may be thought of as the tensor product of cases (1) and (2). Suppose $\lambda^{(1)}: \Omega_{1} \rightarrow S\left(\Omega_{1}\right)$ and $\rho^{(2)}: \Omega_{2} \rightarrow S\left(\Omega_{2}\right)$ are arbitrary maps, where $\Omega_{1}=\left\{1, \cdots, N_{1}\right\}, \Omega_{2}=\left\{1, \cdots, N_{2}\right\}$. Again, we assume, for simplicity that $\lambda_{1}^{(0)}=$ $i d$. Define $\Omega=\Omega_{1} \times \Omega_{2}$ and $\lambda, \rho: \Omega \rightarrow S(\Omega)$ by

$$
\begin{aligned}
& \lambda_{\left(i_{1}, i_{2}\right)}\left(j_{1}, j_{2}\right)=\left(\lambda_{i_{1}}^{(1)}\left(j_{1}\right), j_{2}\right), \\
& \rho_{\left(j_{1}, j_{2}\right)}\left(i_{1}, i_{2}\right)=\left(i_{1}, \rho_{j_{2}}^{(2)}\left(i_{2}\right)\right),
\end{aligned}
$$

so $\pi\left(\left(i_{1}, i_{2}\right),\left(j_{1}, j_{2}\right)\right)=\left(\left(i_{1}, \rho_{j_{2}}^{(2)}\left(i_{2}\right)\right),\left(\lambda_{i_{1}}^{(1)}\left(j_{1}\right), j_{2}\right),\right)$; it is easy to verify that $\pi$ is a permutation of $\Omega_{1} \times \Omega_{2}$. The equations defining $\lambda$ and $\rho$ show that

$$
\lambda_{\rho_{\left(i_{1}, i_{2}\right)}\left(j_{1}, j_{2}\right)}\left(k_{1}, k_{2}\right)=\lambda_{\left(j_{1}, j_{2}\right)}\left(k_{1}, k_{2}\right)=\lambda_{\rho_{\left(i_{1}, i_{2}\right)}^{-1}\left(j_{1}, j_{2}\right)}\left(k_{1}, k_{2}\right)
$$


and that

$$
\rho_{\lambda_{\left(i_{1}, i_{2}\right)}\left(j_{1}, j_{2}\right)}\left(k_{1}, k_{2}\right)=\rho_{\lambda_{\left(i_{1}, i_{2}\right)}^{-1}\left(j_{1}, j_{2}\right)}\left(k_{1}, k_{2}\right)=\rho_{\left(j_{1}, j_{2}\right)}\left(k_{1}, k_{2}\right)
$$

for all $\left(i_{1}, i_{2}\right),\left(j_{1}, j_{2}\right),\left(k_{1}, k_{2}\right)$. It follows easily that $\phi=\rho, \lambda=\psi$, and hence from Remark 7 that

$$
L_{\left(i_{1}, i_{2}\right)}^{(k)}=\lambda_{\left(i_{1}, i_{2}\right)}^{-1} \times \lambda_{\left(i_{1}, i_{2}\right)}^{-1} \times \cdots \lambda_{\left(i_{1}, i_{2}\right)}^{-1}, \text { for all } i_{1}, i_{2}, k
$$

Similarly,

$$
\rho_{L_{\left(i_{1}, i_{2}\right)}^{(k)}(\alpha)}=\rho_{\alpha} \text { for all }\left(i_{1}, i_{2}\right) \in \Omega_{1} \times \Omega_{2}, k \geq 1 \text { and } \alpha \in\left(\Omega_{1} \times \Omega_{2}\right)^{k} .
$$

Hence, the $\sim_{k}$-equivalence classes of $\left(\Omega_{1} \times \Omega_{2}\right)^{k}$, as described in Proposition 10, may be identified with the sets $E_{\sigma}=\Omega_{1}^{k} \times\left\{\alpha \in \Omega_{2}^{k}: \rho_{\alpha}^{(2)}=\sigma\right\}$ for $\sigma$ in the subgroup $G^{(2)}$ of $S\left(\Omega_{1}\right)$ generated by $\left\{\rho_{j}^{(2)}: j \in \Omega_{2}\right\}$. (In the above, we make the natural identification $\left(\Omega_{1} \times \Omega_{2}\right)^{k} \simeq \Omega_{1}^{k} \times \Omega_{2}^{k}$, and use the fact that $\rho_{\left(\alpha_{1}, \alpha_{2}\right)}=\rho_{\alpha_{2}}^{(2)}$.)

Similarly if we let $G^{(1)}$ be the subgroup of $S\left(\Omega_{1}\right)$ generated by $\left\{\lambda_{i}^{(1)}: i \in \Omega_{1}\right\}$ and let $\pi$ denote the natural representation of $G^{(1)}$ on $\mathbb{C}^{N_{1}}$, we see that the group $G_{k}$ of Proposition 10 may be identified with $\otimes^{k}\left(\pi \otimes i d_{\mathbb{C}^{N_{2}}}\right)\left(G^{(1)}\right)$.

From the preceding two paragraphs and the discussion in cases (1) and (2), we see that in this case, the principal graph $\mathcal{G}$ has the following description : let $\tilde{\mathcal{G}}_{1}$ (resp. $\tilde{\mathcal{G}}_{2}$ ) denote the bipartite graph with the sets of even and odd vertices given by $\tilde{\mathcal{G}}_{1}^{(\epsilon)}=\hat{G}^{(1)} \times\{\epsilon\}$ (resp. $\left.\tilde{\mathcal{G}}_{2}^{(\epsilon)}=G^{(2)} \times\{\epsilon\}\right)$, for $\epsilon=0,1$, and with adjacency matrix given by $\Lambda^{(1)}\left(\left(\pi_{0}, 0\right),\left(\pi_{1}, 1\right)\right)=\left\langle\pi_{0} \otimes \pi, \pi_{1}\right\rangle$ (resp., by $\left.\Lambda^{(2)}\left(\left(g_{0}, 0\right),\left(g_{1}, 1\right)\right)=\#\left\{j \in \Omega_{2}: g_{0} \rho_{j}^{(2)}=\omega g_{1}, \omega \in \mathbb{C},|\omega|=1\right\}\right)$; let $\tilde{\mathcal{G}}$ be the bipartite graph with the sets of even and odd vertices given by $\tilde{\mathcal{G}}^{(\epsilon)}=\tilde{\mathcal{G}}_{1}^{(\epsilon)} \times \tilde{\mathcal{G}}_{2}^{(\epsilon)}$, for $\epsilon=0,1$, and with adjacency matrix given by $\Lambda=\Lambda^{(1)} \otimes \Lambda^{(2)}$ :i.e.,

$$
\Lambda\left(\left(\left(\pi_{0}, 0\right),\left(g_{0}, 0\right)\right),\left(\left(\pi_{1}, 1\right),\left(g_{1}, 1\right)\right)\right)=\Lambda^{(1)}\left(\left(\pi_{0}, 0\right),\left(\pi_{1}, 1\right)\right) \Lambda^{(2)}\left(\left(g_{0}, 0\right),\left(g_{1}, 1\right)\right) .
$$

Finally, $\mathcal{G}$ is the connected component in $\tilde{\mathcal{G}}$ containing $\left(\left(1_{1}, 0\right),\left(1_{2}, 0\right)\right)$, where $1_{1}$ denotes the trivial representation of $G^{(1)}$ and $1_{2}$ denotes the identity element of $G^{(2)}$.

\section{The CASE $N \leq 3$}

Recall from the last paragraph of $\S 2$ that biunitary matrices $u, \tilde{u} \in M_{N}(\mathbb{C}) \otimes$ $M_{N}(\mathbb{C})$ are considered equivalent if there exist unitary matrices $a, b, c, d \in U(N)$ such that $\tilde{u}=(a \otimes b) u(c \otimes d)$. It makes sense, therefore, to call biunitary permutation matrices $u$ and $\tilde{u}$ equivalent if there exist permutation matrices $a, b, c, d$ in $M_{N}(\mathbb{C})$ such that $\tilde{u}=(a \otimes b) u(c \otimes d)$. In terms of the corresponding elements $\pi \leftrightarrow(\rho, \lambda), \tilde{\pi} \leftrightarrow(\tilde{\rho}, \tilde{\lambda}) \in P_{N}$, it is not hard to see that the above relation takes the following form.

Definition 11. If $(\rho, \lambda),(\tilde{\rho}, \tilde{\lambda}) \in P_{N}$, say that $(\rho, \lambda) \sim(\tilde{\rho}, \tilde{\lambda})$ if and only if there exist permutations $\mu_{1}, \mu_{2}, \nu_{1}, \nu_{2} \in S\left(\Omega_{N}\right)$ such that $\tilde{\lambda}_{i}=\mu_{1} \circ \lambda_{\nu_{1}(i)} \circ \nu_{2}$ and $\tilde{\rho}_{j}=\mu_{2} \circ \rho_{\nu_{2}(j)} \circ \nu_{1}$ for all $i, j$ in $\Omega_{N}$.

By the way in which this equivalence was arrived at, it is clear that equivalent elements of $P_{N}$ yield conjugate subfactors. In order to understand the partition of $P_{N}$ into the equivalence classes given by the above definition, we proceed as follows: 
Suppose $(\rho, \lambda) \in P_{N}$. Suppose there are $l$ (resp., $r$ ) distinct permutations in the set $\left\{\lambda_{1}, \cdots \lambda_{N}\right\}$ (resp., $\left\{\rho_{1}, \cdots \rho_{N}\right\}$ ); call these permutations $\xi_{1}, \cdots, \xi_{l}$ (resp., $\left.\eta_{1}, \cdots, \eta_{r}\right)$, say. Let $D_{j}=\left\{i \in \Omega_{N}: \lambda_{i}=\xi_{j}\right\}$ for $1 \leq j \leq l$, and $E_{j}=\{i \in$ $\left.\Omega_{N}: \rho_{i}=\eta_{j}\right\}$ for $1 \leq j \leq r$. We shall write $\lambda=\sum_{j=1}^{l} 1_{D_{j}}(\cdot) \xi_{j}$ and $\rho=\sum_{j=1}^{r} 1_{E_{j}}(\cdot) \eta_{j}$, and call $\left(\# D_{1}, \cdots, \# D_{l}\right)$ and $\left(\# E_{1}, \cdots \# E_{r}\right)$ the partitions of $N$ induced by $\lambda$ and $\rho$ respectively.

Lemma 12. Let $(\rho, \lambda) \in P_{N}$, and let $\lambda=\sum_{j=1}^{l} 1_{D_{j}}(\cdot) \xi_{j}$ and $\rho=\sum_{j=1}^{r} 1_{E_{j}}(\cdot) \eta_{j}$ as above. Then,

(i) if $(\tilde{\rho}, \tilde{\lambda}) \in P_{N}$ and $\left.(\rho, \lambda)\right) \sim(\tilde{\rho}, \tilde{\lambda})$ and if

$$
\tilde{\lambda}=\sum_{j=1}^{\tilde{l}} 1_{\tilde{D}_{j}}(\cdot) \tilde{\xi}_{j} \quad \text { and } \quad \tilde{\rho}=\sum_{j=1}^{\tilde{r}} 1_{\tilde{E}_{j}}(\cdot) \tilde{\eta}_{j}
$$

are the corresponding decompositions of $\tilde{\lambda}$ and $\tilde{\rho}$, then $\tilde{l}=l, \tilde{r}=r$, and $\left(\# \tilde{D}_{1}, \cdots \# \tilde{D}_{l}\right)\left(\operatorname{resp} .\left(\# \tilde{E}_{1}, \cdots \# \tilde{E}_{r}\right)\right)$ is a permutation of $\left(\# \tilde{D}_{1}, \cdots \# \tilde{D}_{l}\right)$ (resp. $\left.\left(\# \tilde{E}_{1}, \cdots \# \tilde{E}_{r}\right)\right)$; and

(ii) if

$$
\Omega_{N}=\coprod_{j=1}^{l} \tilde{D}_{j}=\coprod_{j=1}^{r} \tilde{E}_{j}
$$

are any two partitions of $\Omega_{N}$ such that $\# \tilde{D}_{j}=\# D_{j}$ and $\# \tilde{E}_{j}=\# E_{j}$, then there exists $(\tilde{\rho}, \tilde{\lambda}) \in P_{N}$ such that $(\rho, \lambda) \sim(\tilde{\rho}, \tilde{\lambda}), \tilde{\lambda}=\sum_{j=1}^{l} 1_{\tilde{D}_{j}}(\cdot) \tilde{\xi}_{j}$ and $\tilde{\rho}=\sum_{j=1}^{r} 1_{\tilde{E}_{j}}(\cdot) \tilde{\eta}_{j}$, where $\left\{\tilde{\xi}_{1}, \cdots, \tilde{\xi}_{l}\right\}$ and $\left\{\tilde{\eta}_{1}, \cdots \tilde{\eta}_{r}\right\}$ are some two sets of distinct elements such that $\tilde{\xi}_{1}=\tilde{\eta}_{1}=i d_{\Omega_{N}}$.

Proof. (i) If $\mu_{1}, \mu_{2}, \nu_{1}, \nu_{2}$ are as in Definition 11, then, after a possible re-labelling, $\tilde{D}_{j}=\nu_{1}^{-1}\left(D_{j}\right), \tilde{\xi}_{j}=\mu_{1} \circ \xi_{j} \circ \nu_{2}, \tilde{E}_{j}=\nu_{2}^{-1}\left(E_{j}\right)$, and $\tilde{\eta}_{j}=\mu_{2} \circ \eta_{j} \circ \nu_{1}$.

(ii) Pick permutations $\nu_{1}, \nu_{2} \in S\left(\Omega_{N}\right)$ such that $\nu_{1}\left(\tilde{D}_{j}\right)=D_{j}$ and $\nu_{2}\left(\tilde{E}_{j}\right)=E_{j}$. Put $\mu_{1}=\nu_{2}^{-1} \circ \xi_{1}^{-1}$ and $\mu_{2}=\nu_{1}^{-1} \circ \eta_{1}^{-1}$. Now define $\tilde{\lambda}_{i}=\mu_{1} \circ \lambda_{\nu_{1}(i)} \circ \nu_{2}, \tilde{\rho}=\mu_{2} \circ$ $\rho_{\nu_{2}(j)} \circ \nu_{1}$, and note that this $(\tilde{\rho}, \tilde{\lambda})$ does the job.

Corollary 13. If $P_{N}^{0}=\left\{(\rho, \lambda) \in P_{N}: \lambda_{1}=\rho_{1}=i d_{\Omega_{N}}\right\}$, then: (i) if $(\rho, \lambda) \in$ $P_{N}$, there exists $(\tilde{\rho}, \tilde{\lambda}) \in P_{N}^{0}$ such that $(\rho, \lambda) \sim(\tilde{\rho}, \tilde{\lambda})$; in fact, we may choose $(\tilde{\rho}, \tilde{\lambda})$ so that $\#\left\{i: \tilde{\lambda_{i}}=i d_{\Omega_{N}}\right\} \geq \#\left\{i: \tilde{\lambda}_{i}=\sigma\right\}$ and $\#\left\{i: \tilde{\rho}_{i}=i d_{\Omega_{N}}\right\} \geq \#\{i$ : $\left.\tilde{\rho}_{i}=\sigma\right\}$ for all $\sigma \in S\left(\Omega_{N}\right)$; (ii) if $(\rho, \lambda),(\tilde{\rho}, \tilde{\lambda}) \in P_{N}^{0}$, then $(\rho, \lambda) \sim(\tilde{\rho}, \tilde{\lambda})$ if and only if there exist permutations $\nu_{1}, \nu_{2} \in S\left(\Omega_{N}\right)$ such that $\tilde{\lambda}_{i}=\nu_{2}^{-1} \circ \lambda_{\nu_{1}(1)}^{-1} \circ \lambda_{\nu_{1}(i)} \circ \nu_{2}$ and $\tilde{\rho}_{j}=\nu_{1}^{-1} \circ \rho_{\nu_{2}(1)}^{-1} \circ \rho_{\nu_{2}(j)} \circ \nu_{1}$ for all $i, j$ in $\Omega_{N}$.

Proof. (i) is an immediate consequence of Lemma 12(ii)

As for (ii), if $\mu_{1}, \mu_{2}, \nu_{1}, \nu_{2} \in S\left(\Omega_{N}\right)$ are as in Definition 11, the assumptions $\lambda_{1}=\tilde{\lambda_{1}}=\rho_{1}=\tilde{\rho_{1}}=i d$ imply that $i d=\mu_{1} \circ \lambda_{\nu_{1}(1)} \circ \nu_{2}=\mu_{2} \circ \rho_{\nu_{2}(1)} \circ \nu_{1}$ and hence $\mu_{1}=\nu_{2}^{-1} \circ \lambda_{\nu_{1}(1)}^{-1}$ and $\mu_{2}=\nu_{1}^{-1} \circ \rho_{\nu_{2}(1)}^{-1}$.

In view of the corollary, we shall henceforth restrict ourselves to $P_{N}^{0}$ rather than $P_{N}$ and think of the equations in Corollary 13(ii) as the definition of the equivalence.

In the sequel, if $(\rho, \lambda) \in P_{N}$ we shall use the notation $\lambda=\left(\lambda_{1}, \lambda_{2} \cdots, \lambda_{N}\right)$ and $\rho=\left(\rho_{1}, \rho_{2}, \cdots, \rho_{N}\right)$. It is easy to see that $P_{2}^{0}$ consists of precisely three elements, 
which, in the above notation, may be written as :

(a) $\lambda=(i d, i d)=\rho$;

(b) $\lambda=(i d,(12)), \rho=(i d, i d)$;

(c) $\lambda=(i d, i d), \rho=(i d,(12))$.

All these three examples are covered by cases (1) and (2) of $\S 5$, and so we find that the only principal graphs arising from permutation biunitary matrices in $M_{2}(\mathbb{C}) \otimes M_{2}(\mathbb{C})$ are given in Figure A.

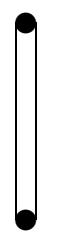

(a)

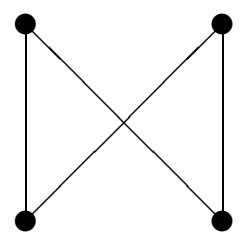

(b) and (c)

Figure A

In fact, Lemma 12 shows that the three elements of $P_{2}^{0}$ listed above are pairwise inequivalent, although the subfactors for examples (b) and (c) yield the same principal graph.

Remark 14. Suppose $(\rho, \lambda) \in P_{N}^{0}$; suppose $\nu \in S\left(\Omega_{N}\right)$ and $\tilde{\lambda}_{i}=\nu^{-1} \circ \lambda_{i} \circ \nu$ for all $i$ in $\Omega_{N}$. Then, $\exists \tilde{\rho}_{j} \in S\left(\Omega_{N}\right), j \in \Omega_{N}$ such that $(\rho, \lambda) \sim(\tilde{\rho}, \tilde{\lambda}) \in P_{N}^{0}$. (Reason: in the notation of Corollary 13(ii), let $\nu_{2}=\nu, \nu_{1}=i d$, and $\tilde{\rho}_{j}=\rho_{\nu(1)}^{-1} \circ \rho_{\nu(j)}$.)

We assume, in the rest of this section, that $N=3$.

Lemma 15. Let $(\rho, \lambda) \in P_{3}$. Then $\exists(\tilde{\rho}, \tilde{\lambda}) \in P_{3}^{0}$ such that $(\rho, \lambda) \sim(\tilde{\rho}, \tilde{\lambda})$ and $\tilde{\lambda}$ is one of the following:

(i) $\tilde{\lambda}=(i d, i d, i d)$;

(ii) $\tilde{\lambda}=(i d, i d,(12))$;

(iii) $\tilde{\lambda}=($ id, id,,$(123))$;

(iv) $\tilde{\lambda}=(i d,(12),(13))$

(v) $\tilde{\lambda}=(i d,(123),(132))$.

Furthermore, the five possibilities above are mutually exclusive.

Proof. Let $\gamma$ be the partition of 3 induced by $\lambda$ (in the sense described in the lines preceding Lemma 12). We consider the three possibilities, $\gamma=(3), \gamma=(2,1), \gamma=$ $(1,1,1)$.

Case (i) : $\gamma=(3)$.

In this case, if $\tilde{\lambda}=(i d, i d, i d)$, it follows from Corollary 13(i) that there exists $\tilde{\rho}$ such that $(\rho, \lambda) \sim(\tilde{\rho}, \tilde{\lambda})$ and $(\tilde{\rho}, \tilde{\lambda}) \in P_{3}^{0}$.

Case (ii) : $\gamma=(2,1)$.

Again, it follows from Corollary 13 (i) that there exists $\left(\rho^{\prime}, \lambda^{\prime}\right) \in P_{3}^{0}$ such that $(\rho, \lambda) \sim\left(\rho^{\prime}, \lambda^{\prime}\right)$, where $\lambda^{\prime}=(i d, i d, \sigma)$ for some $\sigma \in S_{3} \backslash\{i d\}$. Appeal now to Remark 14 to deduce that there exists $(\tilde{\rho}, \tilde{\lambda}) \in P_{3}^{0}$ such that $(\tilde{\rho}, \tilde{\lambda}) \sim\left(\rho^{\prime}, \lambda^{\prime}\right)$ and $\tilde{\lambda}=(i d, i d,(12))$ or $(i d, i d,(123))$ according as $\sigma$ is an odd or even permutation in $S_{3} \backslash\{i d\}$. 
Case (iii) : $\gamma=(1,1,1)$.

As before, we may assume that $\lambda=\left(i d, \lambda_{1}, \lambda_{2}\right)$ with $\lambda_{1} \neq \lambda_{2}$ and $\lambda_{i} \neq i d$ for $i=1,2$. Now consider three subcases depending upon the number $k=\#\{i \in$ $\{1,2\}: \lambda_{i}$ is an odd permutation $\}$. If $k=0$, then $\left\{\lambda_{1}, \lambda_{2}\right\}=\{(123),(132)\}$ and an application of Remark 14 shows that possibility (v) of the lemma occurs. If $k=2$, one application of Remark 14 shows that there exists $\left(\rho^{\prime}, \lambda^{\prime}\right) \in P_{3}^{0}$ such that $(\rho, \lambda) \sim\left(\rho^{\prime}, \lambda^{\prime}\right)$ and $\lambda^{\prime}=(i d,(12), \tau)$ where $\tau=(13)$ or (23). Since $(12)(23)(12)=(13)$, we may (by another application, if necessary, of Remark 14) assume without loss of generality that $\tau=(13)$; i.e., the possibility (iv) of the lemma occurs. If $k=1$, a similar argument to the one used in the case $k=2$, but now using the fact that $(12)(123)(12)=(132)$, shows that there exists $\left(\rho^{\prime}, \lambda^{\prime}\right) \in P_{3}^{0}$ such that $(\rho, \lambda) \sim\left(\rho^{\prime}, \lambda^{\prime}\right)$ and $\lambda^{\prime}=(i d,(12),(123))$. Now set $\nu_{1}=\nu_{2}=(12)$ and define

$$
\tilde{\lambda}_{i}=\nu_{2}^{-1} \circ\left(\lambda_{\nu_{1}(1)}^{\prime}\right)^{-1} \circ \lambda_{\nu_{1}(i)}^{\prime} \circ \nu_{2}, \quad \tilde{\rho}_{j}=\nu_{1}^{-1} \circ\left(\rho_{\nu_{2}(1)}^{\prime}\right)^{-1} \circ \rho_{\nu_{2}(j)}^{\prime} \circ \nu_{1} .
$$

Then $(\rho, \lambda) \sim\left(\rho^{\prime}, \lambda^{\prime}\right) \sim(\tilde{\rho}, \tilde{\lambda}),(\tilde{\rho}, \tilde{\lambda}) \in P_{3}^{0}$, and it is seen that $\tilde{\lambda}=(i d,(12),(13))$; thus the case $k=1$ also leads to possibility (iv) of the lemma.

Thus we have shown that the five possibilities are exhaustive; to prove that they are mutually exclusive, we need to show that if $(\rho, \lambda),(\tilde{\rho}, \tilde{\lambda}) \in P_{3}^{0}$, if $\lambda \neq \tilde{\lambda}$ and if $\lambda, \tilde{\lambda} \in\{(i d, i d, i d),(i d, i d,(12),(i d, i d,(123)),(i d,(12),(13)),(i d,(123),(132))\}$, then $(\rho, \lambda)$ is not equivalent to $(\tilde{\rho}, \tilde{\lambda})$. In view of Lemma $12(\mathrm{i})$, we only need to consider two possibilities :

(a) $\lambda=(i d, i d,(12)), \tilde{\lambda}=(i d, i d,(123))$;

(b) $\lambda=(i d,(12),(13)), \tilde{\lambda}=(i d,(123),(132))$.

The desideratum is a consequence of the observation that, in general, if $(\rho, \lambda)$, $(\tilde{\rho}, \tilde{\lambda}) \in P_{3}^{0}$, if $(\rho, \lambda) \sim(\tilde{\rho}, \tilde{\lambda})$, and if all the $\lambda_{i}$ 's are even permutations, the same must be true of all the $\tilde{\lambda_{i}}$ 's.

We are finally ready to describe the equivalence classes in $P_{3}^{0}$.

Proposition 16. Any element of $P_{3}$ is equivalent to one and only one from among the following list of 18 elements of $P_{3}^{0}$ :
(1) $\lambda=(i d, i d, i d)$
$\rho \in\{(i d, i d, i d),(i d, i d,(12)),(i d, i d,(123))$,
(2) $\lambda=(i d, i d,(12))$
$(i d,(12),(13)),(i d,(123),(132))\}$;
$\rho \in\{(i d, i d, i d),(i d, i d,(12)),(i d, i d,(13))$,
(3) $\lambda=(i d, i d,(123)), \quad \rho \in\{(i d, i d, i d),(i d, i d,(12))\}$;
(4) $\lambda=(i d,(12),(13)), \quad \rho \in\{(i d, i d, i d),(i d, i d,(12)),(i d,(13),(12))\}$;
(5) $\lambda=(i d,(123),(132)), \quad \rho \in\{(i d, i d, i d),(i d,(132),(123))\}$.

Proof. In view of Lemma 15, it suffices to prove that if $(\rho, \lambda) \in P_{3}^{0}$, and if $\lambda$ belongs to the set $\{(i d, i d, i d),(i d, i d,(12)),(i d, i d,(123)),(i d,(12),(13)),(i d,(123),(132))\}$, then there exists a unique $\tilde{\rho}$ such that:

(a) $(\rho, \lambda) \sim(\tilde{\rho}, \lambda)$ and (b) $(\tilde{\rho}, \lambda)$ satisfies one of the conditions $(1)-(5)$ above.

(1) If $\lambda=(i d, i d, i d)$, the proof of the assertion is exactly like the proof of Lemma 15, with $\rho$ in place of $\lambda$ of that proof. One only needs to observe, further, that if $(\rho, \lambda) \sim(\tilde{\rho}, \tilde{\lambda}),(\rho, \lambda),(\tilde{\rho}, \tilde{\lambda}) \in P_{3}^{0}$ and $\lambda=(i d, i d, i d)$, then necessarily $\tilde{\lambda}=\lambda$ (because of Lemma 12). 
(2) Suppose $\lambda=(i d, i d,(12))$. In this proof, and in the sequel, we shall sometimes denote an element $\pi \in P_{N}^{0}$ by an $N \times N$ matrix with $(i, j)$-th entry $\pi(i, j)$. (Note that in order for a permutation $\pi \in S\left(\Omega_{N}^{2}\right)$ to be biunitary, it is necessary and sufficient that its matrix representation has the following features: along the $i$ th row (resp., $j$ th column), the second (resp., first) coordinates yield a permutation of $\Omega_{N}$, namely $\lambda_{i}$ (resp., $\left.\rho_{j}\right)$.) Thus, if $\pi \leftrightarrow(\rho, \lambda) \in P_{3}^{0}$, with $\lambda=(i d, i d,(12))$ we see that $\pi$ has the form

$$
\pi=\left[\begin{array}{lll}
11 & * 2 & * 3 \\
21 & * 2 & * 3 \\
32 & * 1 & * 3
\end{array}\right] .
$$

Since $\pi \in S\left(\Omega_{3} \times \Omega_{3}\right)$, we see that the $(3,2)$ - entry of the above matrix must be 31 ; thus $\rho_{2} \in\{i d,(12)\}$ and $\rho_{3}$ can be any element of $S_{3}$.

Suppose now that $(\rho, \lambda) \sim(\tilde{\rho}, \lambda)$. Then there exist $\nu_{1}, \nu_{2}$ as in Corollary 13 (ii), with $\tilde{\lambda}=\lambda$. Then, we find that $\lambda_{i}=\nu_{2}^{-1} \circ \lambda_{\nu_{1}(1)}^{-1} \circ \lambda_{\nu_{1}(i)} \circ \nu_{2}$ and $\tilde{\rho}_{i}=\nu_{1}^{-1} \circ \rho_{\nu_{2}(1)}^{-1} \circ$ $\rho_{\nu_{2}(i)} \circ \nu_{1}$, for $i=1,2,3$. Since $\{1,2\}$ and $\{3\}$ are the 'sets of constancy' of $\lambda$, we find from Lemma 12(i) that necessarily $\nu_{1}(\{1,2\})=\{1,2\}$ and $\nu_{1}(3)=3$, whence $\nu_{1}=i d$ or (12); in particular, (12) $=\lambda_{3}=\nu_{2}^{-1} \circ \lambda_{\nu_{1}(1)}^{-1} \circ \lambda_{\nu_{1}(3)} \circ \nu_{2}=\nu_{2}^{-1}(12) \nu_{2}$, and so $\nu_{2}$ fixes 3, i.e., also $\nu_{2} \in\{i d,(12)\}$. Thus, $(\tilde{\rho}, \lambda) \sim(\rho, \lambda)$ and $(\tilde{\rho}, \lambda) \in P_{3}^{0}$ if and only if $\tilde{\rho}_{i}=\nu_{1}^{-1} \circ \rho_{\nu_{2}(1)}^{-1} \circ \rho_{\nu_{2}(i)} \circ \nu_{1}$ where $\nu_{1}, \nu_{2} \in\{i d,(12)\}$.

This says that for $\lambda=(i d, i d,(12)),(\rho, \lambda) \sim(\tilde{\rho}, \lambda) \in P_{3}^{0}$ if and only if $\tilde{\rho} \in$ $\left\{\rho=\left(i d, \rho_{2}, \rho_{3}\right),\left(i d, \rho_{2}^{-1}, \rho_{2}^{-1} \rho_{3}\right),\left(i d,(12) \rho_{2}(12),(12) \rho_{3}(12)\right),\left(i d,(12) \rho_{2}^{-1}(12)\right.\right.$, (12) $\left.\left.\rho_{2}^{-1} \rho_{3}(12)\right)\right\}$. Recall that $(\rho, \lambda) \in P_{3}^{0}$ if and only if $\rho_{2} \in\{i d,(12)\}$ and $\rho_{3}$ is arbitrary. Deduce finally that :

(a) $\left(\left(i d, i d, \rho_{3}\right), \lambda\right) \sim(\tilde{\rho}, \lambda) \in P_{3}^{0}$

$$
\Leftrightarrow \tilde{\rho}=(i d, i d, \pi) \text {, where } \pi \in\left\{\rho_{3},(12) \rho_{3}(12)\right\}
$$

and

(b) $\left(\left(i d,(12), \rho_{3}\right), \lambda\right) \sim(\tilde{\rho}, \lambda) \in P_{3}^{0}$

$$
\Leftrightarrow \tilde{\rho}=(i d,(12), \sigma) \text {, where } \sigma \in\left\{\rho_{3},(12) \rho_{3}, \rho_{3}(12),(12) \rho_{3}(12)\right\} .
$$

This finishes the proof of Case (2).

(3) Suppose $\lambda=(i d, i d,(123))$. If $\pi \leftrightarrow(\rho, \lambda) \in P_{3}^{0}$, then the matrix representation of $\pi$ has the form

$$
\pi=\left[\begin{array}{lll}
11 & * 2 & * 3 \\
21 & * 2 & * 3 \\
32 & * 3 & * 1
\end{array}\right] .
$$

Since $\pi \in S\left(\Omega_{3} \times \Omega_{3}\right)$, we argue first that the (3,3)-entry must be 31 ; since $\rho_{3} \in$ $S\left(\Omega_{3}\right)$, this implies that $\rho_{3} \in\{i d,(12)\}$; since $\pi \in S\left(\Omega_{3} \times \Omega_{3}\right)$, this implies that the $(3,2)$-entry of $\pi$ must be 33 , whence also $\rho_{2} \in\{i d,(12)\}$.

Next, $(\rho, \lambda) \sim(\tilde{\rho}, \lambda) \in P_{3}^{0}$ if and only if there exist $\nu_{1}, \nu_{2} \in S\left(\Omega_{3}\right)$ as in Lemma 13(ii), with $\tilde{\lambda}=\lambda$. Argue as in Case (2) above; we see that this forces $\nu_{1} \in$ $\{i d,(12)\}$ and $\nu_{2} \in\{i d,(123),(132)\}$ (since no odd permutation commutes with (123)). Thus $(\rho, \lambda) \sim(\tilde{\rho}, \lambda) \in P_{3}^{0}$ if and only if $\tilde{\rho}_{i}=\nu_{1}^{-1} \circ \rho_{\nu_{2}(1)}^{-1} \circ \rho_{\nu_{2}(i)} \circ \nu_{1}$, where $\nu_{1} \in\{i d,(12)\}$ and $\nu_{2} \in\{i d,(123),(132)\}$.

It is not hard to deduce from this that $((i d, i d,(12)), \lambda) \sim((i d,(12),(i d)), \lambda) \sim$ $((i d,(12),(12)), \lambda)$. This finishes the proof of Case (3).

(4) Suppose $\lambda=(i d,(12),(13))$. We then find, arguing as in Case (3) above, that $(\rho, \lambda) \in P_{3}^{0}$ if and only if $\rho_{1}=i d, \rho_{2} \in\{i d,(13)\}$ and $\rho_{3} \in\{i d,(12)\}$; i.e., there are 
only four possibilities: $\rho \in\{(i d, i d, i d),(i d,(13), i d),(i d, i d,(12)),(i d,(13),(12))\}$. Of these, we find, by Lemma 12, that the first, third and fourth possibilities are mutually exclusive; on the other hand, the second and third possibilities are equivalent, since if $\rho=(i d,(13), i d)$ and $\tilde{\rho}=(i d, i d,(12))$, and if we put $\nu_{1}=\nu_{2}=(23)$, we see that $\lambda_{i}=\nu_{2}^{-1} \circ \lambda_{\nu_{1}(1)}^{-1} \circ \lambda_{\nu_{1}(i)} \circ \nu_{2}$ and $\tilde{\rho}_{i}=\nu_{1}^{-1} \circ \rho_{\nu_{2}(1)}^{-1} \circ \rho_{\nu_{2}(i)} \circ \nu_{1}$ for all $i$.

(5) $\lambda=(i d,(123),(132))$.

In this case we find that $(\rho, \lambda) \in P_{3}^{0} \Leftrightarrow \rho \in\{(i d, i d, i d),(i d,(132),(123))\}$ and the two possibilities are inequivalent by Lemma 12(i).

For the sake of convenience of reference, we list the 18 inequivalent cases below; in each case, we write the matrix representations of $\pi$ and $\pi^{-1}$; the description of the matrix for $\pi^{-1}$ immediately yields the $\phi$ and $\psi$ in each case - and we observe the striking fact that in the first 17 of the 18 cases, we have $\lambda=\psi, \phi=\rho$ ! Further, in those cases where $\lambda_{i}=i d_{\Omega_{3}}$ for all $i$ or $\rho_{j}=i d_{\Omega_{3}}$ for all $j$, we also display the principal graph (as per the analysis of $\S 5$ ). Other principal graphs will be given later.

(1)

$$
\pi=\pi^{-1}=\left[\begin{array}{lll}
11 & 12 & 13 \\
21 & 22 & 23 \\
31 & 32 & 33
\end{array}\right]
$$

$\lambda=(i d, i d, i d)=\psi, \rho=(i d, i d, i d)=\phi$. Principal graph:

$(2)$

\section{Figure 1}

$$
\pi=\left[\begin{array}{ccc}
11 & 12 & 23 \\
21 & 22 & 13 \\
31 & 32 & 33
\end{array}\right]=\pi^{-1}
$$

$\lambda=(i d, i d, i d), \rho=(i d, i d,(12)) ; \phi^{-1}=(i d, i d,(12)), \psi^{-1}=(i d, i d, i d)$. Principal graph:

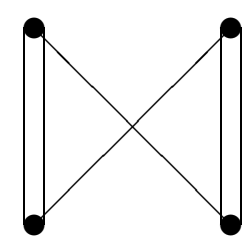

FiguRE 2

$$
\pi=\left[\begin{array}{lll}
11 & 12 & 23 \\
21 & 22 & 33 \\
31 & 32 & 13
\end{array}\right] ; \pi^{-1}=\left[\begin{array}{lll}
11 & 12 & 33 \\
21 & 22 & 13 \\
31 & 32 & 23
\end{array}\right]
$$


$\lambda=(i d, i d, i d), \rho=(i d, i d,(123)) ; \phi^{-1}=(i d, i d,(132)), \psi^{-1}=(i d, i d, i d)$. Principal graph:

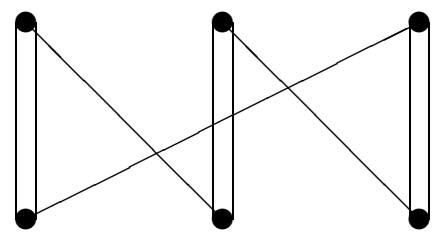

$(4)$

FiguRe 3

$$
\pi=\left[\begin{array}{ccc}
11 & 22 & 33 \\
21 & 12 & 23 \\
31 & 32 & 13
\end{array}\right]=\pi^{-1}
$$

$\lambda=(i d, i d, i d), \rho=(i d,(12),(13)) ; \phi^{-1}=(i d,(12),(13)), \psi^{-1}=(i d, i d, i d)$. Principal graph:

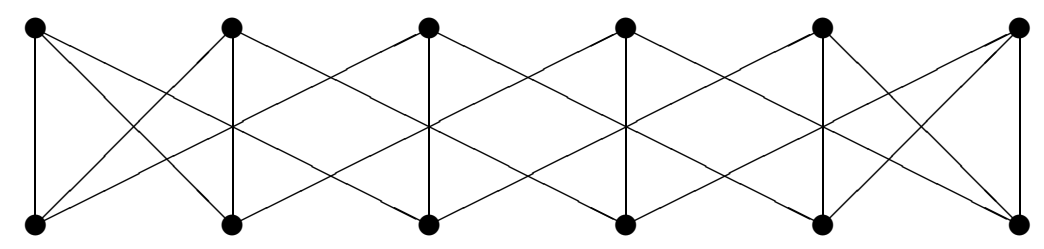

FiguRE 4

$(5)$

$$
\pi=\left[\begin{array}{lll}
11 & 22 & 33 \\
21 & 32 & 13 \\
31 & 12 & 23
\end{array}\right] ; \pi^{-1}=\left[\begin{array}{lll}
11 & 32 & 23 \\
21 & 12 & 33 \\
31 & 32 & 13
\end{array}\right]
$$

$\lambda=(i d, i d, i d), \rho=(i d,(123),(132)) ; \phi^{-1}=(i d,(132),(123)), \psi^{-1}=(i d, i d, i d)$. Principal graph:

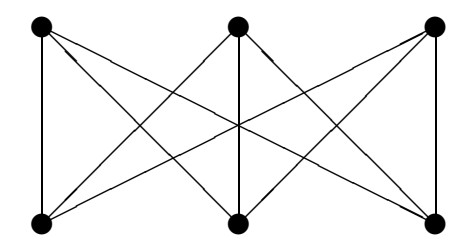

FiguRE 5

(6)

$$
\pi=\left[\begin{array}{lll}
11 & 12 & 13 \\
21 & 22 & 23 \\
32 & 31 & 33
\end{array}\right]=\pi^{-1}
$$

$\lambda=(i d, i d,(12)), \rho=(i d, i d, i d) ; \phi^{-1}=(i d, i d, i d), \psi^{-1}=(i d, i d,(12))$. Principal graph: same as Case (2) 
(7)

$$
\pi=\pi^{-1}=\left[\begin{array}{lll}
11 & 12 & 23 \\
21 & 22 & 13 \\
32 & 31 & 33
\end{array}\right]
$$

$\lambda=\rho=\phi^{-1}=\psi^{-1}=(i d, i d,(12))$.

(8)

$$
\pi=\left[\begin{array}{ccc}
11 & 12 & 33 \\
21 & 22 & 23 \\
32 & 31 & 13
\end{array}\right]=\pi^{-1}
$$

$\lambda=(i d, i d,(12)), \rho=(i d, i d,(13)) ; \phi^{-1}=(i d, i d,(13)), \psi^{-1}=(i d, i d,(12))$.

(9)

$$
\pi=\left[\begin{array}{lll}
11 & 12 & 23 \\
21 & 22 & 33 \\
32 & 31 & 13
\end{array}\right] ; \pi^{-1}=\left[\begin{array}{lll}
11 & 12 & 33 \\
21 & 22 & 13 \\
32 & 31 & 23
\end{array}\right] ;
$$

$\lambda=(i d, i d,(12)), \rho=(i d, i d,(123)) ; \phi^{-1}=(i d, i d,(132)), \psi^{-1}=(i d, i d,(12))$.

(10)

$$
\pi=\left[\begin{array}{ccc}
11 & 22 & 13 \\
21 & 12 & 23 \\
32 & 31 & 33
\end{array}\right]=\pi^{-1}
$$

$\lambda=(i d, i d,(12)), \rho=(i d,(12), i d) ; \phi^{-1}=(i d,(12), i d), \psi^{-1}=(i d, i d,(12))$.

$$
\pi=\left[\begin{array}{ccc}
11 & 22 & 33 \\
21 & 12 & 23 \\
32 & 31 & 13
\end{array}\right]=\pi^{-1}
$$

$\lambda=(i d, i d,(12)), \rho=(i d,(12),(13)) ; \phi^{-1}=(i d,(12),(13)), \psi^{-1}=(i d, i d,(12))$.

$$
\pi=\left[\begin{array}{lll}
11 & 12 & 13 \\
21 & 22 & 23 \\
32 & 33 & 31
\end{array}\right] ; \pi^{-1}=\left[\begin{array}{lll}
11 & 12 & 13 \\
21 & 22 & 23 \\
33 & 31 & 32
\end{array}\right] ;
$$

$\lambda=(i d, i d,(123)), \rho=(i d, i d, i d) ; \phi^{-1}=(i d, i d, i d), \psi^{-1}=(i d, i d,(132))$.

Principal graph: same as Case (3)

(13)

$$
\pi=\left[\begin{array}{lll}
11 & 12 & 23 \\
21 & 22 & 13 \\
32 & 33 & 31
\end{array}\right] ; \pi^{-1}=\left[\begin{array}{lll}
11 & 12 & 23 \\
21 & 22 & 13 \\
33 & 31 & 32
\end{array}\right] ;
$$

$\lambda=(i d, i d,(123)), \rho=(i d, i d,(12)) ; \phi^{-1}=(i d, i d,(12)), \psi^{-1}=(i d, i d,(132))$.

(14)

$$
\pi=\left[\begin{array}{ccc}
11 & 12 & 13 \\
22 & 21 & 23 \\
33 & 32 & 31
\end{array}\right]=\pi^{-1}
$$

$\lambda=(i d,(12),(13)), \rho=(i d, i d, i d) ; \phi^{-1}=(i d, i d, i d), \psi^{-1}=(i d,(12),(13))$. Principal graph: 


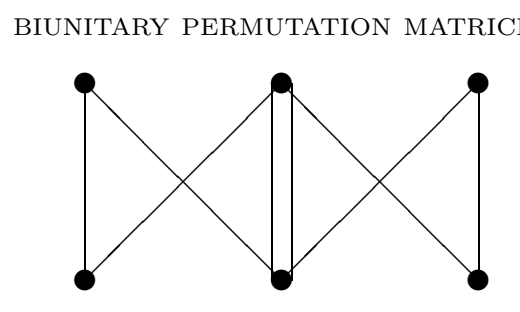

4711

FigURE 14

$(15)$

$$
\pi=\left[\begin{array}{ccc}
11 & 12 & 23 \\
22 & 21 & 13 \\
33 & 32 & 31
\end{array}\right]=\pi^{-1}
$$

$\lambda=(i d,(12),(13)), \rho=(i d, i d,(12)) ; \phi^{-1}=(i d, i d,(12)), \psi^{-1}=(i d,(12),(13))$.

(16)

$$
\pi=\left[\begin{array}{lll}
11 & 32 & 23 \\
22 & 21 & 13 \\
33 & 12 & 31
\end{array}\right]=\pi^{-1}
$$

$\lambda=(i d,(12),(13)), \rho=(i d,(13),(12)) ; \phi^{-1}=(i d,(13),(12)), \psi^{-1}=(i d,(12)$,

$$
\pi=\left[\begin{array}{lll}
11 & 12 & 13 \\
22 & 23 & 21 \\
33 & 31 & 32
\end{array}\right] ; \pi^{-1}=\left[\begin{array}{lll}
11 & 12 & 13 \\
23 & 21 & 22 \\
32 & 33 & 31
\end{array}\right] ;
$$

$\lambda=(i d,(123),(132)), \rho=(i d, i d, i d) ; \phi^{-1}=(i d, i d, i d), \psi^{-1}=(i d,(132),(123))$. Principal graph: same as Case (5)

(18)

$$
\pi=\left[\begin{array}{lll}
11 & 32 & 23 \\
22 & 13 & 31 \\
33 & 21 & 12
\end{array}\right] ; \pi^{-1}=\left[\begin{array}{lll}
11 & 33 & 22 \\
32 & 21 & 13 \\
23 & 12 & 31
\end{array}\right]
$$

$\lambda=(i d,(123),(132)), \rho=(i d,(132),(123)) ; \phi^{-1}=((23),(13),(12)), \psi^{-1}=$ ((23), (12), (13)).

We conclude this section with some useful facts about a special class of biunitary permutation matrices. (Some of these facts are true for more general permutation biunitary matrices, but we will not need that here.)

Proposition 17. Assume $(\rho, \lambda) \in P_{N}$ satisfies $\lambda_{i}=\lambda_{i}^{-1}=\psi_{i}, \rho_{i}=\rho_{i}^{-1}=\phi_{i}$ for all $i \in \Omega_{N}$. Then the following hold:

(a) $L_{x}^{(k)}(\alpha)=\left(\lambda_{x}\left(\alpha_{1}\right), \lambda_{\rho_{\alpha_{1}}(x)}\left(\alpha_{2}\right), \lambda_{\rho_{\alpha_{2}} \rho_{\alpha_{1}}(x)}\left(\alpha_{3}\right), \cdots, \lambda_{\rho_{\alpha_{k-1}} \cdots \rho_{\alpha_{1}}(x)}\left(\alpha_{k}\right)\right)$ for all $x \in \Omega_{N}, k \in \mathbb{N}$ and $\alpha \in \Omega_{N}^{k}$; in particular, if $(\alpha, \gamma) \in \Omega_{N}^{k+l}$ where $\alpha \in \Omega_{N}^{k}$ and $\gamma \in \Omega_{N}^{l}$, then, for all $x \in \Omega_{N}$,

$$
L_{x}^{(k+l)}(\alpha, \gamma)=\left(L_{x}^{(k)}(\alpha), L_{\rho_{\alpha}^{-1}(x)}^{(l)}(\gamma)\right) .
$$

(b) $L_{x}^{(k)^{2}}=i d_{\Omega_{N}^{k}}$ for all $x \in \Omega_{N}^{k}$. 
(c) If $\alpha, \beta \in \Omega_{N}^{k}$ and $\sim_{k}$ is the equivalence relation considered in Proposition 10 then

$\alpha \sim_{k} \beta \Leftrightarrow \rho_{L_{x_{1}}^{(k)} L_{x_{2}}^{(k)} \ldots L_{x_{n}}^{(k)}(\alpha)}=\rho_{L_{x_{1}}^{(k)} L_{x_{2}}^{(k)} \ldots L_{x_{n}}^{(k)}(\beta)}$ for all $n \in \mathbb{N}, x_{1}, x_{2}, \cdots, x_{n} \in \Omega_{N}$.

(d) If $\alpha \in \Omega_{N}^{k}$, then $(\alpha, a, a) \sim_{k+2}(\alpha, b, b)$ for all $a, b \in \Omega_{N}$.

(e) If $\alpha, \beta \in \Omega_{N}^{k}$ and $\gamma \in \Omega_{N}^{l}$, then

$$
\alpha \sim_{k} \beta \Leftrightarrow(\alpha, \gamma) \sim_{k+l}(\beta, \gamma) \Leftrightarrow(\gamma, \alpha) \sim_{l+k}(\gamma, \beta) .
$$

Proof. (a) Begin by recalling (cf. Remark 7) that the hypothesis implies that, for $k \in \mathbb{N}, \alpha \in \Omega_{N}^{k}, a, x \in \Omega_{N}$, we have

$$
L_{x}^{(k+1)}(\alpha, a)=\left(L_{x}^{(k)}(\alpha), \lambda_{\rho_{\alpha}^{-1}(x)}(a)\right) ;
$$

it follows easily by induction that

$$
L_{x}^{(k)}(\alpha)=\left(\lambda_{x}\left(\alpha_{1}\right), \lambda_{\rho_{\alpha_{1}}(x)}\left(\alpha_{2}\right), \cdots, \lambda_{\rho_{\alpha_{k-1}} \rho_{\alpha_{k-2}} \cdots \rho_{\alpha_{1}}(x)}\left(\alpha_{k}\right)\right) ;
$$

this equation is easily seen to imply that if $\alpha \in \Omega_{N}^{k}, \gamma \in \Omega_{N}^{l}$ and $x \in \Omega_{N}$, then

$$
L_{x}^{(k+l)}(\alpha, \gamma)=\left(L_{x}^{(k)}(\alpha), L_{\rho_{\alpha}^{-1}(x)}^{(l)}(\gamma)\right) .
$$

(b) Since $L_{x}^{(1)}=\lambda_{x}^{-1}=\lambda_{x}$, it is clear that $L_{x}^{(1)^{2}}=i d_{\Omega_{N}} \forall x \in \Omega_{N}$. Suppose we have shown that $L_{x}^{(k)^{2}}=i d_{\Omega_{N}^{k}}$. Then, it follows from equation 9 that, for $\alpha \in \Omega_{N}^{k}$ and $a, x \in \Omega_{N}$, we have

$$
L_{x}^{(k+1)^{2}}(\alpha, a)=L_{x}^{(k+1)}\left(L_{x}^{(k)}(\alpha), \lambda_{\rho_{\alpha}^{-1}(x)}(a)\right)=\left(L_{x}^{(k)^{2}}(\alpha), \lambda_{\rho_{L_{x}^{(k)}(\alpha)}^{-1}(x)} \lambda_{\rho_{\alpha}^{-1}(x)}(a)\right)
$$

appeal now to Proposition 6(iii) (which says that $\rho_{L_{x}^{(k)}(\alpha)}^{-1}(x)=\phi_{\alpha}^{-1}(x)=\rho_{\alpha}^{-1}(x)$ ) the induction hypothesis and the assumption that $\lambda_{i}{ }^{2}=i d_{\Omega_{N}}$ for all $i \in \Omega_{N}$ to deduce that, indeed, $L_{x}^{(k+1)^{2}}=i d_{\Omega_{N}^{k+1}}$.

(c) By definition of the equivalence relation $\sim_{k}$ (and (b) above), we have, for $\alpha, \beta \in \Omega_{N}^{k}$,

$\alpha \sim_{k} \beta \Leftrightarrow \rho_{L_{x_{1}}^{(k)} L_{x_{2}}^{(k)} \cdots L_{x_{2 n+1}}^{(k)}(\alpha)}=\rho_{L_{x_{1}}^{(k)} L_{x_{2}}^{(k)} \cdots L_{x_{2 n+1}}^{(k)}(\beta)} \forall n \in \mathbb{N}, x_{1}, \cdots, x_{2 n+1} \in \Omega_{N}$.

On the other hand, we noticed in the proof of Lemma 8 that $\alpha \sim_{k} \beta \Rightarrow \phi_{\alpha}=\phi_{\beta}$. Also if $x_{1}, x_{2}, \cdots x_{2 n} \in \Omega_{N}$, the definition of $\sim_{k}$ and (b) above show that

$$
\alpha \sim_{k} \beta \Rightarrow L_{x_{1}}^{(k)} \cdots L_{x_{2 n}}^{(k)}(\alpha) \sim L_{x_{1}}^{(k)} \cdots L_{x_{2 n}}^{(k)}(\beta) ;
$$

hence $\alpha \sim_{k} \beta \Rightarrow \phi_{L_{x 1}^{(k)} \cdots L_{x_{2 n}}^{(k)}(\alpha)}=\phi_{L_{x_{1}}^{(k)} \ldots L_{x_{2 n}}^{(k)}(\beta)} \forall x_{1}, x_{2} \cdots, x_{2 n} \in \Omega_{N}$. Since $\phi=\rho$, this finishes the proof of (c).

(d) Two applications of equation 9 show that, for all $\alpha \in \Omega_{N}^{k}, a, x \in \Omega_{N}$,

$$
L_{x}^{(k+2)}(\alpha, a, a)=\left(L_{x}^{(k)}(\alpha), \lambda_{\rho_{\alpha}^{-1}(x)}(a), \lambda_{\rho_{a} \rho_{\alpha}^{-1}(x)}(a)\right) ;
$$

but since $\lambda=\psi$ and $\rho=\phi$, it follows from Proposition 3(iii) that

$$
\lambda_{\rho_{a} \rho_{\alpha}^{-1}(x)}(a)=\lambda_{\phi_{a}^{-1}\left(\rho_{\alpha}^{-1}(x)\right)}^{-1}(a)=\psi_{\rho_{\alpha}^{-1}(x)}^{-1}(a)=\lambda_{\rho_{\alpha}^{-1}(x)}(a) ;
$$

hence

$$
L_{x}^{(k+2)}(\alpha, a, a)=\left(L_{x}^{(k)}(\alpha), \lambda_{\rho_{\alpha}^{-1}(x)}(a), \lambda_{\rho_{\alpha}^{-1}(x)}(a)\right) .
$$


Thus, if $\alpha \in \Omega_{N}^{k}$ and $a \in \Omega_{N}$, we find that, for all $x \in \Omega_{N}, L_{x}^{(k+2)}(\alpha, a, a)$ has the form $\left(L_{x}^{(k)}(\alpha), a^{\prime}, a^{\prime}\right)$ for some $a^{\prime}$ in $\Omega_{N}$, which implies, in particular, that $\rho_{L_{x}^{(k+2)}(\alpha, a, a)}=\rho_{L_{x}^{(k)}(\alpha)}$. Repeated application of the above fact shows that $\rho_{L_{x_{1}}^{(k+2)} L_{x_{2}}^{(k+2)} \cdots L_{x_{n}}^{(k+2)}(\alpha, a, a)}=\rho_{L_{x_{1}}^{(k)} L_{x_{2}}^{(k)} \cdots L_{x_{n}}^{(k)}(\alpha)}$ for arbitrary $x_{1}, \cdots, x_{n} \in \Omega_{N}$ and $n \in \mathbb{N}$. This proves $(\mathrm{d})$, since $\rho_{L_{x_{1}}^{(k+2)} \ldots L_{x_{n}}^{(k+2)}(\alpha, a, a)}$ is independent of $a$.

(e) Suppose first that $\alpha \sim_{k} \beta$. Then, by (a), we have

$$
L_{x}^{(k+l)}(\alpha, \gamma)=\left(L_{x}^{(k)}(\alpha), L_{\rho_{\alpha}^{-1}(x)}^{(l)}(\gamma)\right),
$$

so that $\rho_{L_{x}^{(k+l)}(\alpha, \gamma)}=\rho_{L_{x}^{(k)}(\alpha)} \rho_{L_{\rho_{\alpha}^{-1}(x)}^{(l)}(\gamma)}$; but $\alpha \sim_{k} \beta \Rightarrow \rho_{\alpha}=\phi_{\alpha}=\phi_{\beta}=\rho_{\beta}$; thus, $\alpha \sim_{k} \beta \Rightarrow L_{x}^{(k+1)}(\alpha, \gamma)=\left(L_{x}^{(k)}(\alpha), \gamma^{\prime}\right)$ and $L_{x}^{(k+l)}(\beta, \gamma)=\left(L_{x}^{(k)}(\beta), \gamma^{\prime}\right)$ for some $\gamma^{\prime}$, and $\rho_{L_{x}^{(k+l)}(\alpha, \gamma)}=\rho_{L_{x}^{(k+l)}(\beta, \gamma)}$. It follows that if $n \in \mathbb{N}$ and $x_{1}, \cdots x_{n} \in \Omega_{N}$, then

$$
L_{x_{1}}^{(k+l)} \cdots L_{x_{n}}^{(k+l)}(\alpha, \gamma)=\left(L_{x_{1}}^{(k)} \cdots L_{x_{n}}^{(k)}(\alpha), \tilde{\gamma}\right)
$$

and

$$
L_{x_{1}}^{(k+l)} \cdots L_{x_{n}}^{(k+l)}(\beta, \gamma)=\left(L_{x_{1}}^{(k)} \cdots L_{x_{n}}^{(k)}(\beta), \tilde{\gamma}\right),
$$

for some $\tilde{\gamma}$, and hence $(\alpha, \gamma) \sim_{k+l}(\beta, \gamma)$. In a similar manner, we have, for any $x \in \Omega_{N}, L_{x}^{(k+l)}(\gamma, \alpha)=\left(L_{x}^{(l)}(\gamma), L_{\rho_{\gamma}^{-1}(x)}^{(k)}(\alpha)\right)$, and we may deduce that $(\gamma, \alpha) \sim_{l+k}$ $(\gamma, \beta)$.

Now for the more important cancellation assertion of the reverse implication. To start with, if $(\alpha, \gamma) \sim_{k+l}(\beta, \gamma)$, then $\rho_{\alpha} \rho_{\gamma}=\rho_{(\alpha, \gamma)}=\rho_{(\beta, \gamma)}=\rho_{\beta} \rho_{\gamma}$, whence $\rho_{\alpha}=\rho_{\beta}$; hence, for $x$ in $\Omega_{N}$, we see that if $\gamma^{\prime}=L_{\rho_{\alpha}^{-1}(x)}(\gamma)=L_{\rho_{\beta}^{-1}(x)}(\gamma)$, then $L_{x}^{(k+l)}(\alpha, \gamma)=\left(L_{x}^{(k)}(\alpha), \gamma^{\prime}\right)$ and $L_{x}^{(k+l)}(\beta, \gamma)=\left(L_{x}^{(k)}(\beta), \gamma^{\prime}\right)$; the assumpion $(\alpha, \gamma) \sim_{k+l}(\beta, \gamma)$ implies that $\rho_{L_{x}^{(k+l)}(\alpha, \gamma)}=\rho_{L_{x}^{(k+l)}(\beta, \gamma)}$ and hence, as before, that $\rho_{L_{x}^{(k)}(\alpha)}=\rho_{L_{x}^{(k)}(\beta)}$. It follows easily that $\rho_{L_{x_{1}}^{(k)} \ldots L_{x_{n}}^{(k)}(\alpha)}=\rho_{L_{x_{1}}^{(k)} \ldots L_{x_{n}}^{(k)}(\beta)}$ for all $x_{1}, \cdots, x_{n} \in \Omega_{N}$, i.e., that $\alpha \sim_{k} \beta$. The proof of left-cancellation, i.e., $(\gamma, \alpha) \sim_{l+k}$ $(\gamma, \beta) \Rightarrow \alpha \sim_{k} \beta$, is similar.

Corollary 18. Suppose $(\rho, \lambda) \in P_{N}$ satisfies the conditions of Proposition 17. Then,

(i) if $\Delta=\left\{(a, a): a \in \Omega_{N}\right\}$, then $L_{x}^{(2)}(\Delta) \subseteq \Delta$ for all $x$ in $\Omega_{N}$;

(ii) if $X_{k}=\left\{\alpha \in \Omega_{N}^{k}: \alpha_{i} \neq \alpha_{i+1}\right.$ for $\left.1 \leq i<k\right\}, k \geq 2$, then $L_{x}^{(k)}\left(X_{k}\right) \subseteq X_{k}$ for all $x$ in $\Omega_{N}$.

Proof. (i) We have

$$
\begin{aligned}
L_{x}^{(2)}(a, a) & =\left(\lambda_{x}(a), \lambda_{\rho_{a}(x)}(a)\right) \\
& =\left(\lambda_{x}(a), \psi_{x}(a)\right) \\
& =\left(\lambda_{x}(a), \lambda_{x}(a)\right) .
\end{aligned}
$$

(ii) Write $\alpha=(\beta, \gamma, \delta)$ where $\beta=\left(\alpha_{1}, \cdots, \alpha_{i-1}\right), \gamma=\left(\alpha_{i}, \alpha_{i+1}\right)$ and $\delta=$ $\left(\alpha_{i+2}, \cdots, \alpha_{k}\right)$. Then $\alpha \in X_{k} \Rightarrow \gamma \notin \Delta \Rightarrow L_{y}^{(2)}(\gamma) \notin \Delta$ for all $y \in \Omega_{N}$ (by (i) above); but $L_{x}^{(k)}(\alpha)=\left(L_{x}^{(i-1)}(\beta), L_{\rho_{\beta}^{-1}(x)}^{(2)}(\gamma), L_{\rho_{\gamma}^{-1} \rho_{\beta}^{-1}(x)}^{(k-1)}(\delta)\right)$; hence $L_{x}^{(k)}(\alpha)$ has distinct coordinates at places $i$ and $i+1$. Since $i$ was arbitrary, this shows that $L_{x}^{(k)}\left(X_{k}\right) \subseteq X_{k}$. 


\section{The Finite PRINCIPAL Graphs, When $N=3$}

In the last section, we obtained 18 special permutation biunitary matrices, when $N=3$, such that every permutation biunitary matrix, when $N=3$, is equivalent to one from among these 18. Also, the principal graphs of the cases numbered $1-6,12,14$ and 17 were described. In this section, we compute the principal graphs of the cases numbered $7-10,13$ and 18, and show that the graphs are finite. (We also observe, at the end of this section, a sufficient condition, for general $N$, for the principal graph to be finite.) In the next and final section, we show that two of the remaining cases - namely, cases 11 and 15 - correspond to infinite depth subfactors, and give some idea of what the principal graphs look like. About the last remaining case, numbered 16 in our list, we say nothing, as we know nothing beyond the first two relative commutants in that case.

As is to be expected, all the computations are based on Proposition 10, and we shall use the following notation in the rest of the paper: for $k=1,2, \cdots$, we write $\tilde{G_{k}}$ (resp. $G_{k}$ ) for the subgroup of $S\left(\Omega_{N}^{k}\right)$ generated by $\left\{L_{x}^{(k)}: x \in \Omega_{N}\right\}$ (resp. $\left\{L_{x}^{(k)^{-1}} L_{y}^{(k)}: x, y \in \Omega_{N}\right\}$ ); for $\alpha, \beta$ in $\Omega_{N}^{k}$, we write $\alpha \sim_{k} \beta$ if and only if $\rho_{L_{x}^{(k)}(\sigma(\alpha))}=\rho_{L_{x}^{(k)}(\sigma(\beta))}$ for all $x$ in $\Omega_{N}$ and $\sigma$ in $G_{k}$; finally, for $\alpha$ in $\Omega_{N}^{k}$, we write $H_{[\alpha]}=\left\{\sigma \in G_{k}: \sigma([\alpha])=[\alpha]\right\}$, where $[\alpha]=\left\{\beta \in \Omega_{N}^{k}: \beta \sim_{k} \alpha\right\}$.

Case (8) : $\lambda=(i d, i d,(12))=\psi, \rho=(i d, i d,(13))=\phi$.

This case is covered by Proposition 17. Note first that $\rho_{x}(2)=2$ for all $x \in \Omega_{3}$, hence also $\rho_{\alpha}(2)=2$ for all $\alpha \in \Omega_{3}^{k}, k \geq 1$; since $\lambda_{2}=i d$, this implies - cf. Proposition $17(\mathrm{a})$ - that $L_{2}^{(k)}=i d_{\Omega_{3}^{k}}$, and hence $\tilde{G}_{k}=G_{k}$.

Note next that $\lambda_{x}(3)=3$ for all $x$; it follows that if $\alpha \in \Omega_{3}^{k}$ and $\alpha=\left(w_{1}, 3, w_{2}\right.$, $3, \cdots)$, where $w_{1}, w_{2}, \cdots$ are (possibly empty) words in 1 and 2 , then - since clearly $\rho_{\gamma}=(13)^{l_{3}(\gamma)}$ for all $\gamma \in \Omega_{N}^{m}$, where $l_{3}(\gamma)=\#\left\{i: 1 \leq i \leq m, \gamma_{i}=3\right\}-$ it follows that

$$
L_{1}^{(k)}(\alpha)=\left(w_{1}, 3, \tilde{w}_{2}, 3, w_{3}, 3, \tilde{w}_{4}, 3, \cdots\right)
$$

and

$$
L_{3}^{(k)}(\alpha)=\left(\tilde{w}_{1}, 3, w_{2}, 3, \tilde{w}_{3}, 3, w_{4}, 3, \cdots\right),
$$

where $\tilde{w}_{i}$ is obtained by changing each 1 (resp. 2) in $w_{i}$ to 2 (resp. 1). It follows that $L_{1}^{(k)}, L_{3}^{(k)}$ and $L_{1}^{(k)} L_{3}^{(k)}$ are all elements of order 2, and hence that $G_{k}=\left\{i d_{\Omega_{3}^{k}}, L_{1}^{(k)}, L_{3}^{(k)}, L_{1}^{(k)} L_{3}^{(k)}\right\} \cong \mathbb{Z}_{2} \times \mathbb{Z}_{2}$ for all $k \geq 2$ (and $G_{1} \cong \mathbb{Z}_{2}$ ).

The above description of the $L_{x}^{(k)}$ 's shows that $l_{3}\left(L_{x}^{(k)}(\alpha)\right)=l_{3}(\alpha)$ for all $x$, whence $\rho_{\alpha}=\rho_{L_{x}^{(k)}(\alpha)}=\rho_{\sigma(\alpha)} \forall \sigma \in G_{k}$; it follows that $\alpha \sim_{k} \beta \Leftrightarrow l_{3}(\alpha) \equiv l_{3}(\beta)$ $(\bmod 2)$, and hence $\Omega_{3}^{k}$ splits into two equivalence classes $E_{k}$ and $O_{k}$, where $E_{k}$ (resp. $O_{k}$ ) is the set of those $\alpha$ 's in $\Omega_{3}^{k}$ with an even (resp. odd) number of 3's appearing in it. It should be clear that $E_{k+1}=\left(E_{k} \times\{1,2\}\right) \amalg\left(O_{k} \times\{3\}\right)$ and $O_{k+1}=\left(O_{k} \times\{1,2\}\right) \amalg\left(E_{k} \times\{3\}\right)$.

Further, the above description of $L_{x}^{(k)}, x=1,3$, shows that

$$
\begin{aligned}
& \left.L_{1}^{(k+1)}\right|_{E_{k+1}}=L_{1}^{(k)} \times i d,\left.\quad L_{3}^{(k+1)}\right|_{E_{k} \times\{1,2\}}=L_{3}^{(k)} \times(12), \\
& \left.L_{3}^{(k+1)}\right|_{O_{k} \times\{3\}}=L_{3}^{(k)} \times i d,\left.\quad L_{1}^{(k+1)}\right|_{O_{k} \times\{1,2\}}=L_{1}^{(k)} \times(12) \text {, } \\
& \left.L_{1}^{(k+1)}\right|_{\left.\right|_{k} \times\{3\}}=L_{1}^{(k)} \times i d,\left.\quad L_{3}^{(k+1)}\right|_{O_{k+1}}=L_{3}^{(k)} \times i d .
\end{aligned}
$$


(In particular, each of the equivalence classes $E_{k}$ and $O_{k}$ is invariant under $G_{k}$, and so $H_{[\alpha]}=G_{k}$ for all $\alpha \in \Omega_{3}^{k}$.)

Note now that if $\pi$ is a representation of $G_{k}$ on an inner product space $V$, if we write $V_{\epsilon_{1}, \epsilon_{3}}=\left\{v \in V: \pi\left(L_{1}^{(k)}\right) v=\epsilon_{1} v, \pi\left(L_{3}^{(k)}\right) v=\epsilon_{3} v\right\}$ for $\epsilon_{1}, \epsilon_{3} \in\{1,-1\}$ and if we let $p_{\epsilon_{1}, \epsilon_{3}}$ denote the orthogonal projection onto $V_{\epsilon_{1}, \epsilon_{3}}$, then $\left\{p_{\epsilon_{1}, \epsilon_{3}}: \epsilon_{1}, \epsilon_{3} \in\right.$ $\{1,-1\}\}$ is a set of minimal central projections in $\pi\left(G_{k}\right)^{\prime}$.

The foregoing remarks, together with Proposition 10, are seen fairly easily to imply that the Bratteli diagram for the inclusion of $C_{k}$ in $C_{k+1}$ (where $C_{k}$ embeds in $C_{k+1}$ via $\left.x \mapsto x \otimes 1\right)$ is given, when $k \geq 2$, by the following:

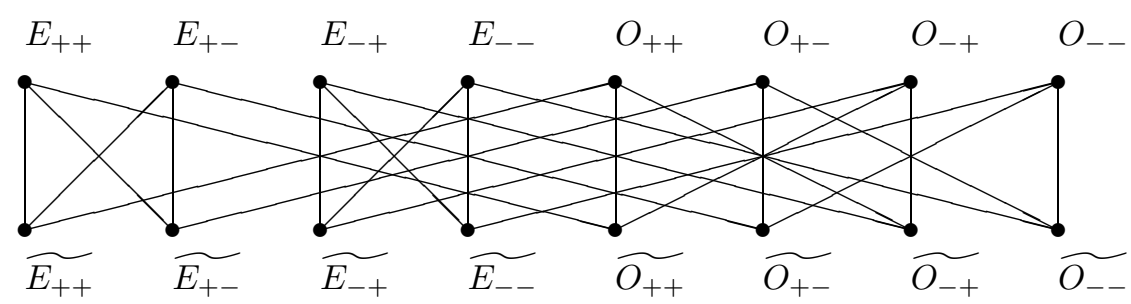

Figure 8

We may now conclude that the above graph is the principal graph for the subfactor, since: (a) it has norm 3, and (b) it should be a subgraph of the principal graph.

Case (7) : $\lambda=\rho=\psi=\phi=(i d, i d,(12))$.

This case is also covered by Proposition 17. Note first that $\rho_{x}(3)=3$ for all $x$ in $\Omega_{3}$; hence also $\rho_{\alpha}(3)=3$ and $\rho_{\alpha}\{1,2\}=\{1,2\}$ for all $\alpha \in \Omega_{3}^{k} k \geq 1$; since $\lambda_{1}=\lambda_{2}=i d$ and $\lambda_{3}=(12)$, it follows that $L_{1}^{(k)}=L_{2}^{(k)}=i d$ and $L_{3}^{(k)}=(12) \times(12) \times \cdots \times(12)(k$ terms $)$. Hence $G_{k}=\tilde{G}_{k}=\left\{1, L_{3}^{(k)}\right\} \cong \mathbb{Z}_{2}$ for all $k$.

Also, $\rho_{\alpha}=(12)^{l_{3}(\alpha)}$ for $\alpha \in \Omega_{3}^{k}$, where $l_{3}(\alpha)$ is as before; it follows from the above description of $G_{k}$ that also $\rho_{\alpha}=\rho_{\sigma(\alpha)}$ for all $\sigma$ in $G_{k}$. Hence $\alpha \sim_{k} \beta \Leftrightarrow$ $l_{3}(\alpha) \equiv l_{3}(\beta)(\bmod 2)$.

As in Case 8, we see that there are precisely two equivalence classes $E_{k}$ and $O_{k}$ in $\Omega_{3}^{k}$, these sets having the same description as before. Further, each of $E_{k}$ and $O_{k}$ is stable under $G_{k}$; we have $E_{k+1}=\left(E_{k} \times\{1,2\}\right) \amalg\left(O_{k} \times\{3\}\right)$ and $O_{k+1}=$ $\left(E_{k} \times\{3\}\right) \amalg\left(O_{k} \times\{1,2\}\right)$; also, $L_{3}^{(k+1)}=L_{3}^{(k)} \times(12)$.

Since any unitary representation $\pi$ of $G_{k}$ on $V$ has two isotypical subrepresentations corresponding to the subspaces given by $V_{ \pm}=\left\{v \in V: \pi\left(L_{3}^{(k)}\right) v= \pm v\right\}$, we may deduce, as in case 7 , that the principal graph is given, in this case, by Figure 7.

Case $(10): \lambda=(i d, i d,(12))=\psi, \rho=(i d,(12), i d)=\phi$.

This case is also covered by Proposition 17. Begin by noting that $\rho_{x}(3)=3$ for all $x$; hence $\rho_{\alpha}(3)=3$ for all $\alpha$, and so $L_{3}^{(k)}=(12) \times(12) \times \cdots(12)(k$ terms). Also $\rho_{\alpha}\{1,2\}=\{1,2\}$ for all $\alpha$ and $\lambda_{1}=\lambda_{2}=i d$, which implies that $L_{1}^{(k)}=L_{2}^{(k)}=i d$. Hence, again, $G_{k}=\tilde{G}_{k}=\left\{i d, L_{3}^{(k)}\right\}$. Also $\rho_{\alpha}=(12)^{l_{2}(\alpha)}$, where, as before, we set $l_{x}(\alpha)=\#\left\{i: \alpha_{i}=x\right\}$; on the other hand, $\rho_{L_{3}^{(k)}(\alpha)}=(12)^{l_{1}(\alpha)}$; we conclude that $\alpha \sim_{k} \beta \Leftrightarrow l_{1}(\alpha) \equiv l_{1}(\beta)(\bmod$ 


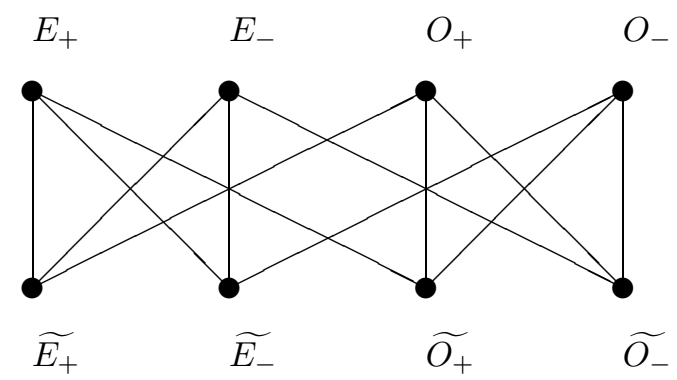

FiguRE 7

2) and $l_{2}(\alpha) \equiv l_{2}(\beta)(\bmod 2)$. Hence, $\Omega_{3}^{k}$ splits into four equivalence classes $E_{i j}^{(k)}$, $i, j \in\{0,1\}$, where $E_{i j}^{(k)}=\left\{\alpha \in \Omega_{3}^{k}: l_{1}(\alpha) \equiv i(\bmod 2), l_{2}(\alpha) \equiv j(\bmod 2)\right\}$. Notice that $E_{00}^{(k)}$ and $E_{11}^{(k)}$ are stable under $G_{k}$ whereas $G_{k}$ maps $E_{01}^{(k)}$ to $E_{10}^{(k)}$ and vice versa. It follows that

$$
H_{[\alpha]}= \begin{cases}G_{k}, & \text { if } \alpha \in E_{00}^{(k)} \cup E_{11}^{(k)}, \\ (1), & \text { if } \alpha \in E_{10}^{(k)} \cup E_{01}^{(k)} .\end{cases}
$$

Notice next that

$$
E_{i j}^{(k+1)}=\left(E_{i \dot{+} 1, j}^{(k)} \times\{1\}\right) \amalg\left(E_{i, j+1}^{(k)} \times\{2\}\right) \amalg\left(E_{i j}^{(k)} \times\{3\}\right),
$$

where $\dot{+}$ denotes addition modulo 2 . Since $L_{3}^{(k+1)}=L_{3}^{(k)} \times(12)$, and since the groups in question are $\mathbb{Z}_{2}$ and (1), we may deduce, as in the earlier cases, that the principal graph is given thus:

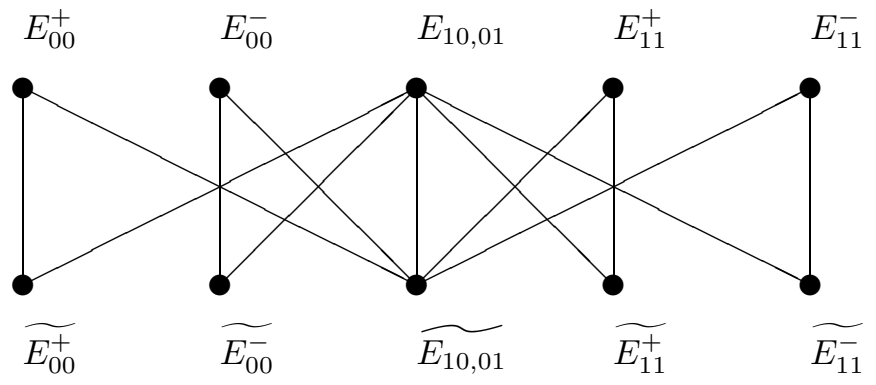

FIGURE 10

Case (18): $\lambda=\rho^{-1}=(i d,(123),(132)), \phi=((23),(13),(12)), \psi=((23),(12)$, (13)).

First consider the case $k=1$. Since $\rho_{x} \neq \rho_{y}$ for $x \neq y$, we see that $x \sim_{1}$ $y \Leftrightarrow x=y$ for $x, y \in \Omega_{3}$. Further, since $L_{x}^{(1)}=\psi_{x}^{-1}$, we find from the above description of $\psi$ that $G_{1}=A_{3}, \tilde{G}_{1}=S_{3}$. Since $G_{1}$ acts transitively on $\Omega_{1}$, it follows that in this case, $C_{1}=\mathbb{C}$.

Next, letting $k=2$, use the definitions and find, after a small computation, that, in terms of their decompositions into disjoint cycles, the permutations $L_{x}^{(2)}$ of $\Omega_{3}^{2}$ are given by

$$
\begin{aligned}
& L_{1}^{(2)}=((2,1)(3,2))((2,2)(3,3))((2,3)(3,1)), \\
& L_{2}^{(2)}=((1,1)(2,2))((1,2)(2,3))((1,3)(2,1))
\end{aligned}
$$


and

$$
L_{3}^{(2)}=((1,1)(3,3))((1,2)(3,1))((1,3)(3,2)) .
$$

If we set $\Delta_{0}=\{(1,1),(2,2),(3,3)\}, \Delta_{1}=\{(1,2),(2,3),(3,1)\}$ and $\Delta_{2}=$ $\{(1,3),(2,1),(3,2)\}$, we find that :

(a) $\rho_{\alpha}=(123)^{j}$ if $\alpha \in \Delta_{j}$ for $j=0,1,2$; and

(b) $L_{x}^{(2)}\left(\Delta_{j}\right)=\Delta_{j}$ for all $x \in \Omega_{3}$ and $j=0,1,2$.

It follows easily that $\Delta_{0}, \Delta_{1}, \Delta_{2}$ are the distinct equivalence classes in $\Omega_{3}^{2}$.

Note that $G_{2}$ is the subgroup generated by $\left\{L_{1}^{(2)} L_{2}^{(2)}, L_{2}^{(2)} L_{3}^{(2)}\right\}$. From the above formulae for the $L_{x}^{(2)}$ 's, we find that $L_{1}^{(2)} L_{2}^{(2)}$ and $L_{2}^{(2)} L_{3}^{(2)}$ have cycle decompositions given by

$$
L_{1}^{(2)} L_{2}^{(2)}=L_{2}^{(2)} L_{3}^{(2)}=((1,1)(3,3)(2,2))((1,2)(3,1)(2,3))((1,3)(3,2)(2,1)) .
$$

It follows now from Proposition 10 that $C_{2} \simeq(\mathbb{C} \oplus \mathbb{C} \oplus \mathbb{C}) \oplus(\mathbb{C} \oplus \mathbb{C} \oplus \mathbb{C}) \oplus(\mathbb{C} \oplus \mathbb{C} \oplus \mathbb{C})$. Hence the Bratteli diagram for $C_{1} \subseteq C_{2}$ is given by :

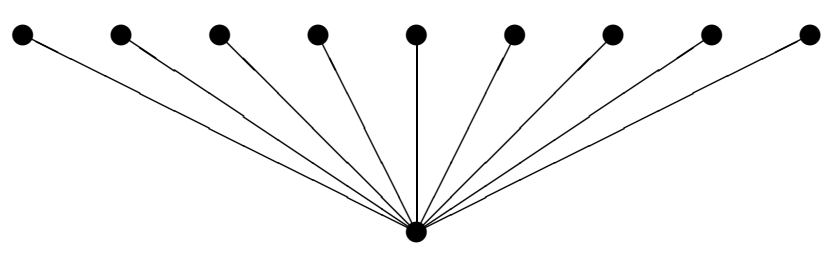

FIGURE 18

Since this graph has norm 3, we see as before that this agrees with the principal graph (except that the vertices at the lower level are now the even vertices).

Case (9): $\lambda=\psi=(i d, i d,(12)), \rho=\phi=(i d, i d,(123))$.

Since $\lambda=\psi$ and $\rho=\phi$, it follows from Remark 7 that $L_{x}^{(k+1)}(\alpha, a)=$ $\left(L_{x}^{(k)}(\alpha), \lambda_{\rho_{\alpha}^{-1}(x)}^{-1}(a)\right)$ for all $k \geq 1, \alpha \in \Omega_{3}^{k}$ and $a, x \in \Omega_{3}$. Since $\lambda_{y}(3)=3$ for all $y$, it follows from the previous equation that if $\alpha \in \Omega_{3}^{k}, l \leq k$ and $\alpha_{l}=3$, then $\left(L_{x}^{(k)}(\alpha)\right)_{l}=3$. Since $\rho_{\alpha}=(123)^{l_{3}(\alpha)}$ with $l_{x}(\alpha)=\#\left\{i: \alpha_{i}=x\right\}$ as before, it follows that $\rho_{L_{x}^{(k)}(\alpha)}=\rho_{\alpha}$ for all $\alpha$ in $\Omega_{3}^{k}$ and $x \in \Omega_{3}$; hence $\rho_{\alpha}=\rho_{\sigma(\alpha)}$ for all $\sigma \in$ $G_{k}$. It follows that $\alpha \sim_{k} \beta$ if and only if $l_{3}(\alpha) \equiv l_{3}(\beta)(\bmod 3)$. Hence, $\Omega_{3}^{k}$ splits into three equivalence classes $\left\{E_{j}^{(k)}: j=0,1,2\right\}$, where $E_{j}^{(k)}=\left\{\alpha \in \Omega_{3}^{k}: l_{3}(\alpha) \equiv j\right.$ $(\bmod 3)\}$; then, if $\alpha \in E_{j}^{(k)}$, it follows that $\rho_{\alpha}=(123)^{j}$; in particular, we also see that $E_{j}^{(k)}=\left\{\alpha \in \Omega_{3}^{k}: \rho_{\alpha}=(123)^{j}\right\}$.

Notice next that if $x, y \in \Omega_{3}$, then, since $\rho_{\sigma(\alpha)}=\rho_{\alpha}$ for all $\sigma$ in $G_{k}$, we have, for $k \geq 1, \alpha \in \Omega_{3}^{k}$ and $a \in \Omega_{3}$,

$$
\begin{aligned}
L_{y}^{(k+1)} L_{x}^{(k+1)}(\alpha, a) & =L_{y}^{(k+1)}\left(L_{x}^{(k)}(\alpha), \lambda_{\rho_{\alpha}^{-1}(x)}^{-1}(a)\right) \\
& =\left(L_{y}^{(k)} L_{x}^{(k)}(\alpha), \lambda_{\rho_{L_{x}^{(k)}(\alpha)}^{-1}(y)}^{-1} \lambda_{\rho_{\alpha}^{-1}(x)}^{-1}(a)\right) \\
& =\left(L_{y}^{(k)} L_{x}^{(k)}(\alpha), \lambda_{\rho_{\alpha}^{-1}(y)}^{-1} \lambda_{\rho_{\alpha}^{-1}(x)}^{-1}(a)\right) ;
\end{aligned}
$$


since $\lambda_{i} \lambda_{j}=\lambda_{j} \lambda_{i}$ for all $i, j$, it follows by induction on $k$ that $L_{x}^{(k)}$ and $L_{y}^{(k)}$ commute. Also, since $\lambda_{i}^{2}=i d_{\Omega_{3}}$ for all $i$, the above equations show , in addition, that $L_{x}^{(k)^{2}}=i d_{\Omega_{3}^{k}}$ for all $k$, and for all $x$. It follows, in particular, that, for all $k, G_{k}=\left\{i d_{\Omega_{3}^{k}}, L_{1}^{(k)} L_{2}^{(k)}, L_{2}^{(k)} L_{3}^{(k)}, L_{3}^{(k)} L_{1}^{(k)}\right\}$ is a group of involutions; it can be seen that when $k=2$, the four elements above are distinct, and hence we see that $G_{k} \simeq \mathbb{Z}_{2} \times \mathbb{Z}_{2}$ for $k \geq 2$.

Notice next that for any $k \geq 1$ and $j=0,1,2$, we have

$$
E_{j}^{(2 k+1)}=\left(E_{j}^{(2 k)} \times\{1,2\}\right) \amalg\left(E_{j+2}^{(2 k)} \times\{3\}\right),
$$

where $\dot{+}$ denotes addition modulo three. Notice too that $\left.L_{x}^{(2 k+1)}\right|_{E_{j}^{(2 k)} \times\{1,2\}}=$ $L_{x}^{(2 k)} \times \lambda_{(123)^{-j}(x)}$ and that $\left.L_{x}^{(2 k+1)}\right|_{E_{j}^{(2 k)} \times\{3\}}=L_{x}^{(2 k)} \times i d$.

If we now use the fact that any representation of $\mathbb{Z}_{2} \times \mathbb{Z}_{2}$ breaks up naturally into four isotypical subrepresentations we may deduce that the Bratteli diagram for the inclusion of $C_{2 k}$ into $C_{2 k+1}$ ( via $x \mapsto x \otimes 1$ ) is given thus:

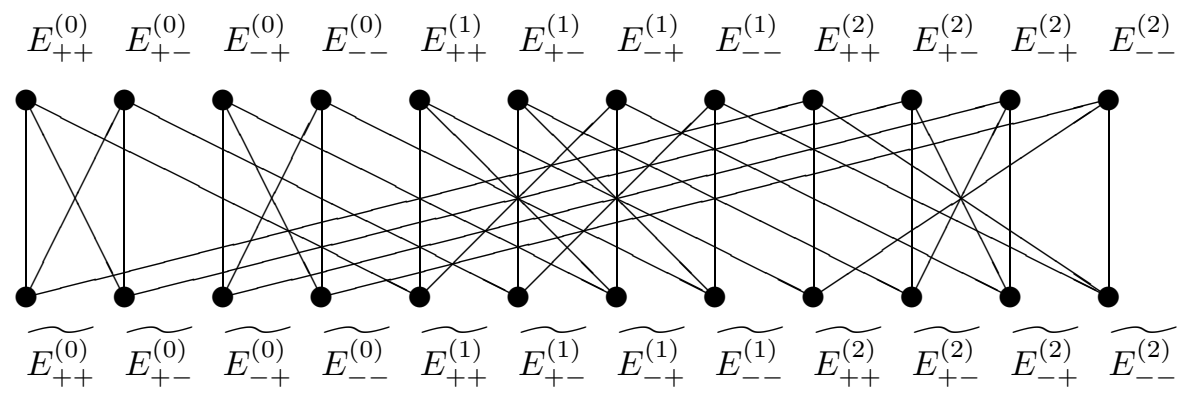

FIGURE 9

(We use the convention here that if $V$ is a representation space for $G_{k}$, then $V_{\epsilon_{1}, \epsilon_{2}}=\left\{v \in V: \pi\left(L_{1}^{(k)} L_{2}^{(k)}\right) v=\epsilon_{1} v\right.$ and $\left.\pi\left(L_{2}^{(k)} L_{3}^{(k)}\right) v=\epsilon_{2} v\right\}$ for $\epsilon_{1}, \epsilon_{2} \in$ $\{ \pm 1\}$.

Case $(13): \lambda=(i d, i d,(123))=\psi, \rho=(i d, i d,(12))=\phi$.

As before, since $\lambda=\psi$ and $\rho=\phi$, we have the inductive formula

$$
L_{x}^{(k+1)}(\alpha, a)=\left(L_{x}^{(k)}(\alpha), \lambda_{\rho_{\alpha}^{-1}(x)}^{-1}(a)\right) .
$$

Notice now that $\rho_{\alpha}(3)=3$ and $\rho_{\alpha}\{1,2\}=\{1,2\}$, whence $\lambda_{\rho_{\alpha}^{-1}(3)}^{-1}=(132)$ and $\lambda_{\rho_{\alpha}^{-1}(x)}^{-1}=i d$ for $x \in\{1,2\}$ and for all $\alpha$; it follows that, for all $k, L_{1}^{(k)}=L_{2}^{(k)}=$ $i d_{\Omega_{3}^{k}}$ and $L_{3}^{(k)}=(132) \times(132) \times \cdots \times(132)(k$ terms $)$, and so

$$
\tilde{G}_{k}=G_{k}=\left\{i d_{\Omega_{3}^{k}}, L_{3}^{(k)}, L_{3}^{(k)^{2}}\right\} .
$$

Note next that $\rho_{\alpha}=(12)^{l_{3}(\alpha)}$ for $\alpha$ in $\Omega_{3}^{k}, k \geq 1$, where, as before, $l_{x}(\alpha)=\#\{i$ : $\left.\alpha_{i}=x\right\}$. Clearly $l_{3}\left(L_{3}^{(k)}(\alpha)\right)=l_{1}(\alpha)$ and $l_{3}\left(L_{3}^{(k)^{2}}(\alpha)\right)=l_{2}(\alpha)$, and so we find that $\alpha \sim_{k} \beta$ if and only if $l_{x}(\alpha) \equiv l_{x}(\beta)(\bmod 2)$ for $x=1,2$, 3. Since $l_{1}(\alpha)+l_{2}(\alpha)+l_{3}(\alpha) \equiv k$, we see that $\alpha \sim_{k} \beta \Leftrightarrow l_{1}(\alpha) \equiv l_{1}(\beta)(\bmod 2)$ and 
$l_{2}(\alpha) \equiv l_{2}(\beta)(\bmod 2)$. Thus, we see that $\Omega_{3}^{k}$ splits into four equivalence classes $E_{i j}^{(k)}, 0 \leq i, j \leq 1$, where $E_{i j}^{(k)}=\left\{\alpha \in \Omega_{3}^{k}: l_{1}(\alpha) \equiv i(\bmod 2), l_{2}(\alpha) \equiv j(\bmod 2)\right\}$.

Since $l_{3}(\alpha) \equiv l_{1}(\alpha)+l_{2}(\alpha)+k(\bmod 2)$ for $\alpha \in \Omega_{3}^{k}$, it follows that $L_{3}^{(k)}\left(E_{i, j}^{(k)}\right)=$ $E_{j, i+j+k}^{(k)}$ (where $\dot{+}$ denotes addition modulo 2). It follows that if $k$ is even, then $E_{00}^{(k)}$ is stable under $G_{k}$ while $L_{3}^{(k)}\left(E_{01}^{(k)}\right)=E_{11}^{(k)}$ and $L_{3}^{(k)}\left(E_{11}^{(k)}\right)=E_{10}^{(k)}$; thus, when $k$ is even, we find that $H_{[\alpha]}$ is $G_{k}$ or $\{1\}$ according as to whether $\alpha \in E_{00}^{(k)}$ or $\alpha \in E_{01}^{(k)} \cup E_{11}^{(k)} \cup E_{10}^{(k)}$. Similarly, if $k$ is odd then $E_{11}^{(k)}$ is stable under $G_{k}$, $L_{3}^{(k)}\left(E_{01}^{(k)}\right)=E_{10}^{(k)}$ and $L_{3}^{(k)}\left(E_{10}^{(k)}\right)=E_{00}^{(k)}$, whence $H_{[\alpha]}$ is $G_{k}$ or $\{1\}$ according as to whether $\alpha \in E_{11}^{(k)}$ or $\alpha \in E_{01}^{(k)} \cup E_{10}^{(k)} \cup E_{00}^{(k)}$.

Notice the obvious identity

$$
E_{i j}^{(2 k+1)}=\left(E_{i j}^{(2 k)} \times\{3\}\right) \amalg\left(E_{i+1, j}^{(2 k)} \times\{1\}\right) \amalg\left(E_{i, j \dot{+1}}^{(2 k)} \times\{2\}\right)
$$

where $\dot{+}$ denotes addition modulo 2 .

Since a typical representation of $\mathbb{Z}_{3}$ splits into three isotypical summands, it is not too hard to see now, in view of the foregoing analysis and Proposition 10, that the Bratteli diagram for the inclusion of $C_{2 k}$ in $C_{2 k+1}$ (via $x \mapsto x \otimes 1$ ) is given, for $k \geq 1$, by:

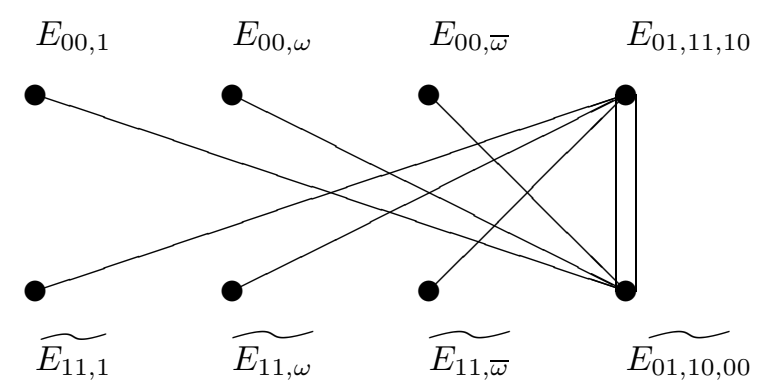

FIGURE 13

Remark 19. Suppose $(\lambda, \rho) \in P_{N}^{0}$, and $G_{k}, \tilde{G}_{k}$ are the subgroups of $S\left(\Omega_{N}^{k}\right)$ discussed earlier. By definition of the $L_{x}^{(k)}$ 's we know that there is a unique epimorphism of $\tilde{G}_{k+1}$ into $\tilde{G}_{k}$ which maps $L_{x}^{(k+1)}$ to $L_{x}^{(k)}$ for all $x$ in $\Omega_{N}$. Let $\tilde{G}$ denote the inverse limit of the sequence $\left\{\tilde{G}_{k}: k \geq 1\right\}$. If it turns out that $\tilde{G}$ is finite, it is not hard to deduce from the definition of $\sim_{k}$ and Proposition 10 that the principal graph must be finite. (In fact, we suspect that the above condition is also a necessary condition for the principal graph to be finite.)

\section{TWO INFINITE-DEPTH SUBFACTORS}

In this section, we discuss the cases numbered 11 and 15 in the list of $\S 6$. We show that in both cases the principal graph is infinite, and give a partial description of the graph in Case 11 and a complete description of the graph in Case 15.

Both these cases are covered by Proposition 17, and hence the conclusions of that proposition are valid in these cases.

We shall find it convenient to work with the limiting case $k=\infty$ in the following sense : for $x$ in $\Omega_{3}$, we consider the transformation $L_{x}$ of the sequence space $\Omega_{3}^{\mathbb{N}}$ defined by the requirement that $\left(L_{x} \alpha\right)_{k]}=L_{x}^{(k)} \alpha_{k]}$, where we use the symbol 
$\beta_{k]}$ to denote the truncation, to the first $k$ coordinates, of the infinite sequence $\beta$. Thus, by Proposition 17 (a), we have

$$
L_{x} \alpha=\left(\lambda_{x}\left(\alpha_{1}\right), \lambda_{\rho_{\alpha_{1}}(x)}\left(\alpha_{2}\right), \cdots, \lambda_{\rho_{\alpha_{k-1}} \cdots \rho_{\alpha_{1}}(x)}\left(\alpha_{k}\right), \cdots\right) .
$$

It follows easily from the fact that each $L_{x}^{(k)}$ is an involution - see Proposition 17 (b) - that $L_{x}^{2}=i d_{\Omega_{3}^{\mathbb{N}}}$ for all $x \in \Omega_{3}$; in particular, $L_{x} \in S\left(\Omega_{3}^{\mathbb{N}}\right)$ for all $x \in \Omega_{3}$. As in the case of finite $k$, we define $\tilde{G}$ (resp., $G$ ) to be the subgroup of $S\left(\Omega_{3}^{\mathbb{N}}\right.$ ) generated by $\left\{L_{x}: x \in \Omega_{3}\right\}$ (resp., $\left\{L_{x} L_{y}: x, y \in \Omega_{3}\right\}$ ).

Before proceeding to a discussion of the cases 11 and 15, we pause to record a simple fact that will be of use in both cases.

Lemma 20. Let $(\lambda, \rho) \in P_{N}^{0}$ satisfy the hypothesis of Proposition 17; then for any $k \geq 1, \alpha \in \Omega_{N}^{k}, a \in \Omega_{N}$, it is the case that $(\alpha, a, a) \sim_{k+2}(a, a, \alpha)$.

Proof. By induction (and the easy half of the cancellation rule - see Proposition 17 (e)), it suffices to consider the case $k=1$; but if $b \in \Omega_{N}$, then by Proposition 17 (d), we have $(b, a, a) \sim_{3}(b, b, b) \sim_{3}(a, a, b)$.

Case (11) : $\lambda=(i d, i d,(12)), \rho=(i d,(12),(13)$.

We break the argument into a sequence of lemmas.

Lemma 21. If $\alpha \in \Omega_{3}^{\mathbb{N}}$ and $l \in \mathbb{N}$, then

$$
\alpha_{l}=3 \Leftrightarrow\left(L_{x} \alpha\right)_{l}=3 \text { for all } x \in \Omega_{3} .
$$

Proof. Since $\lambda_{x}(3)=3$ for all $x \in \Omega_{3}$, this follows from equation (10).

Lemma 22. $\alpha \in \Omega_{3}^{k}, k \geq 1 \Rightarrow(\alpha, 1,2) \sim_{k+2}(\alpha, 2,1)$.

Proof. Let $\{a, b\}=\{1,2\}$. Then, since $\lambda_{\rho_{a}(y)}=\lambda_{y}$ for all $y \in \Omega_{3}$, we have, for any $x \in \Omega_{3}$,

$$
\begin{aligned}
L_{x}^{k+2}(\alpha, a, b) & =\left(L_{x}^{(k)} \alpha, \lambda_{\rho_{\alpha}^{-1}(x)}(a), \lambda_{\rho_{a} \rho_{\alpha}^{-1}(x)}(b)\right) \\
& =\left(L_{x}^{(k)} \alpha, \lambda_{\rho_{\alpha}^{-1}(x)}(a), \lambda_{\rho_{\alpha}^{-1}(x)}(b)\right) ;
\end{aligned}
$$

since $\lambda_{y}\{1,2\}=\{1,2\}$ for all $y \in \Omega_{3}$, we see that $L_{x}^{(k+2)}(\alpha, a, b)=\left(L_{x}^{(k)} \alpha, a^{\prime}, b^{\prime}\right)$ for some $a^{\prime}, b^{\prime}$ such that $\left\{a^{\prime}, b^{\prime}\right\}=\{1,2\}$. It follows that for any $n \geq 1$ and $x_{1}, \cdots, x_{n} \in \Omega_{3}$,

$$
L_{x_{1}}^{(k+2)} L_{x_{2}}^{(k+2)} \cdots L_{x_{n}}^{(k+2)}(\alpha, a, b)=\left(L_{x_{1}}^{k} L_{x_{2}}^{k} \cdots L_{x_{n}}^{k} \alpha, a^{\prime}, b^{\prime}\right)
$$

where $\left\{a^{\prime}, b^{\prime}\right\}=\{a, b\}=\{1,2\}$. Hence, as $\rho_{1} \rho_{2}=\rho_{2} \rho_{1}=(12)$, we see that

$$
\rho_{L_{x_{1}}^{(k+2)} L_{x_{2}}^{(k+2)} \cdots L_{x_{n}}^{(k+2)}(\alpha, a, b)}=\rho_{L_{x_{1}}^{k} L_{x_{2}}^{k} \cdots L_{x_{n}}^{k}(\alpha)} \cdot(12)
$$

and the lemma is proved.

Lemma 23. If $\alpha \in \Omega_{3}^{k}, k \geq 1$, there exists $\alpha^{\prime} \in \Omega_{3}^{k}$ such that $\alpha \sim_{k} \alpha^{\prime}$, and $\alpha^{\prime}$ has the form

$$
\alpha^{\prime}=\left(w_{1}, 3, w_{2}, 3, w_{3}, 3, \cdots\right)
$$

where each $w_{i}$ either is non-existent or is one of the following 'words' involving only 1 and $2: w_{i}=(1)$ or $w_{i}=(2)$ or $w_{i}=(1,2)$ or $w_{i}=(2,1)$; further, if some $w_{i}$ is the 'empty word', then so also is every $w_{j}$ for $1 \leq j \leq i$. 
Proof. Begin by locating all the 3's in $\alpha$ and writing $\alpha$ in the form given for $\alpha^{\prime}$ in equation (11), with each $w_{i}$ being a word involving only 1 and 2 . Then, by Lemma 22 , we may assume that if some $w_{i}$ is non-empty, then without going out of the $\sim_{k}$-equivalence class of $\alpha$ we may assume without loss of generality that $w_{i}$ is a string of $p$ ( say ) 1's followed by a string of $q$ (say) 2's, where $p, q \geq 0$. Then, using Lemma 20, we may even assume that $p, q \leq 1$. (Reason : if $p=3$ for instance, then we may move two 1's all the way to the front, and then those two 1's may be replaced by 3's in view of Proposition 17 (d).)

This shows that $\alpha \sim_{k} \alpha^{\prime}$ where $\alpha^{\prime}$ is as in equation (11), where each $w_{i}$ is either empty or $(1),(2)$ or $(1,2)$. The final assertion, about being able to move all the empty words all the way to the front, is an easy consequence of Lemma 20.

Definition 24. An element $\tilde{\alpha} \in \Omega_{3}^{k}$ will be said to be reduced if $\tilde{\alpha}$ is as in equation (11) where the 'words' $w_{i}$ satisfy the conditions described in Lemma 23.

Two reduced elements will be said to have the same configuration if either of them can be obtained from the other by 'flipping' some of the words to their 'opposites', where the words (1) and (2) are considered to be opposites of one another, as also are the words $(1,2)$ and $(2,1)$.

For example, with $k=6$, the element $\alpha=(1,2,1,3,2,1)$ is not reduced, but it is equivalent to the reduced word $\tilde{\alpha}=(3,3,2,3,1,2)$; the set of reduced words with the same configuration as $\tilde{\alpha}$ is $\{(3,3)\} \times\{1,2\} \times\{3\} \times\{(1,2),(2,1)\}$.

Lemma 25. Let $\alpha \in \Omega_{3}^{k}$ be reduced; suppose there are precisely l non-empty 'words' in $\alpha$; then the set $\{\{\alpha\}\}$ of reduced words with the same configuration as $\alpha$ is a set with precisely $2^{l}$ elements which is invariant under the action of the group $G_{k}$.

Proof. Clearly the set $\{\{\alpha\}\}$ has cardinality $2^{l}$, and it is invariant under the action of $G_{k}$ in view of Lemma 21 .

Lemma 26. . Let $k=2 n+1$, and let $\alpha_{0}=(1,3,1,3, \cdots, 1,3,1)$ be the unique reduced element of $\Omega_{3}^{2 n+1}$ with $(n+1)$ coordinates equal to 1 . Let $X_{2 n+1}=\left\{\left\{\alpha_{0}\right\}\right\}$ be the set of reduced elements of $\Omega_{3}^{2 n+1}$ with the same configuration as $\alpha_{0}$. (Thus $X_{2 n+1}=\left\{\beta \in \Omega_{3}^{2 n+1}: \beta_{l}=3 \Leftrightarrow l\right.$ is even $\}$.) Then,

(a) the cyclic group generated by $L_{2}^{(k)} L_{3}^{(k)}$ acts transitively on $X_{2 n+1}$;

(b) no two distinct elements of $X_{2 n+1}$ are equivalent (with respect to $\sim_{2 n+1}$ );

(c) $\alpha \in \Omega_{3}^{2 n+1}$ and $\alpha \sim_{2 n+1} \beta \in X_{2 n+1} \Rightarrow \alpha=\beta$.

Proof. (a) It follows from the definitions and equation (10) that if $\gamma \in \Omega_{3}^{\mathbb{N}}$ and $x \in \Omega_{3}$, then

$$
L_{x}(1,3, \gamma)=\left(\lambda_{x}(1), 3, L_{(13)(x)}(\gamma)\right)
$$

and

$$
L_{x}(2,3, \gamma)=\left(\lambda_{x}(2), 3, L_{(123)(x)}(\gamma)\right) ;
$$

we write out these equations explicitly in the following table, where the entry in the $i$-th row and $j$-th column is $L_{j}(i, 3, \gamma)$, for $i=1,2, j=1,2,3$ :

\begin{tabular}{|c|c|c|c|}
\hline & $L_{1}$ & $L_{2}$ & $L_{3}$ \\
\hline$(1,3, \gamma)$ & $\left(1,3, L_{3} \gamma\right)$ & $\left(1,3, L_{2} \gamma\right)$ & $\left(2,3, L_{1} \gamma\right)$ \\
\hline$(2,3, \gamma)$ & $\left(2,3, L_{2} \gamma\right)$ & $\left(2,3, L_{3} \gamma\right)$ & $\left(1,3, L_{1} \gamma\right)$ \\
\hline
\end{tabular}


From this table, we deduce the following table which has a similar interpretation:

\begin{tabular}{|c|c|c|c|}
\hline & $L_{1} L_{2}$ & $L_{2} L_{1}$ & $L_{1} L_{3}$ \\
\hline$(1,3, \gamma)$ & $\left(1,3, L_{3} L_{2} \gamma\right)$ & $\left(1,3, L_{2} L_{3} \gamma\right)$ & $\left(2,3, L_{2} L_{1} \gamma\right)$ \\
\hline$(2,3, \gamma)$ & $\left(2,3, L_{2} L_{3} \gamma\right)$ & $\left(2,3, L_{3} L_{2} \gamma\right)$ & $\left(1,3, L_{3} L_{1} \gamma\right)$ \\
\hline
\end{tabular}

\begin{tabular}{|c|c|c|c|}
\hline & $L_{3} L_{1}$ & $L_{2} L_{3}$ & $L_{3} L_{2}$ \\
\hline$(1,3, \gamma)$ & $\left(2,3, L_{1} L_{3} \gamma\right)$ & $\left(2,3, L_{3} L_{1} \gamma\right)$ & $\left(2,3, L_{1} L_{2} \gamma\right)$ \\
\hline$(2,3, \gamma)$ & $\left(1,3, L_{1} L_{2} \gamma\right)$ & $\left(1,3, L_{2} L_{1} \gamma\right)$ & $\left(1,3, L_{1} L_{3} \gamma\right)$ \\
\hline
\end{tabular}

In particular, it follows that if we write $S=\left\{L_{x} L_{y}: x, y \in \Omega_{3}, x \neq y\right\}$ and $X_{2 n}=\left\{\alpha \in \Omega_{3}^{2 n}: \alpha_{l}=3 \Leftrightarrow l\right.$ is even $\}$, then for any $x, y \in \Omega_{3}$ with $x \neq y$, and for any $\alpha \in X_{2 n}$, there exists $\sigma$ in $S$ such that $L_{x} L_{y}(\alpha, \gamma)=\left(L_{x}^{(2 n)} L_{y}^{(2 n)}(\alpha), \sigma(\gamma)\right)$ for all $\gamma$ in $\Omega_{3}^{\mathbb{N}}$. For $\sigma$ in $S$, say $\sigma=L_{p} L_{q}$ with $p \neq q$, we define $w(\sigma)=1_{\{p, q\}}(3)$, i.e., $w(\sigma)$ is 1 if $3 \in\{p, q\}$ and 0 if $3 \notin\{p, q\}$.

We shall prove (a) by establishing, by induction, the more complicated statement below:

$(\mathrm{a})^{\prime}$ : Let $\alpha_{0}^{(2 n+1)}=(1,3,1,3, \cdots, 1,3,1) \in \Omega_{3}^{2 n+1}$; define

$$
\alpha_{j}^{(2 n+1)}=\left(L_{2}^{(2 n+1)} L_{3}^{(2 n+1)}\right)^{j} \alpha_{0}^{(2 n+1)} \text {, }
$$

for $0 \leq j \leq 2^{n+1}$, and write $\alpha_{j}^{(2 n+2)}=\left(\alpha_{j}^{(2 n+1)}, 3\right)$ for $0 \leq j \leq 2^{n+1}$. For $0 \leq j<2^{n+1}$, define $\sigma_{j}^{(n)} \in S$ by the requirement that

$$
\left(L_{2} L_{3}\right)\left(\alpha_{j}^{(2 n+2)}, \gamma\right)=\left(\alpha_{j+1}^{(2 n+2)}, \sigma_{j}^{(n)}(\gamma)\right)
$$

for all $\gamma \in \Omega_{3}^{\mathbb{N}}$. Then,

(i) $\alpha_{j}^{(2 n+1)} \neq \alpha_{l}^{(2 n+1)}$ for $0 \leq j<l<2^{(n+1)}$;

(ii) $\alpha_{2^{n+1}}^{(2 n+2)}=\alpha_{0}^{(2 n+2)}$; and

(iii) $\sum_{0 \leq j<2^{n+1}} w\left(\sigma_{j}^{(n)}\right)$ is an odd integer.

The proof of (a) is by induction on $n$. If $n=0$, then $\alpha_{0}^{(1)}=(1), \alpha_{1}^{(1)}=(2)$ and $\alpha_{2}^{(1)}=(1)$ (since $L_{3}^{(1)}=(12)$ ), and the second table above shows that $\sigma_{0}^{(0)}=L_{3} L_{1}, \sigma_{1}^{(0)}=L_{2} L_{1}$, and so $w\left(\sigma_{0}^{(0)}\right)+w\left(\sigma_{1}^{(0)}\right)=1+0=1$; hence the statement $(a)^{\prime}$ is valid for $n=0$.

Suppose we know that $(a)^{\prime}$ is valid for $n$. Temporarily fix $j, 0 \leq j<2^{n+1}$. It is fairly clear that, for any $\gamma$ in $\Omega_{3}^{\mathbb{N}}$,

$$
\left(L_{2} L_{3}\right)^{2^{n+1}}\left(\alpha_{j}^{(2 n+2)}, \gamma\right)=\left(\alpha_{j}^{(2 n+2)}, \sigma_{j-1}^{(n)} \sigma_{j-2}^{(n)} \cdots \sigma_{0}^{(n)} \sigma_{2^{n+1}-1}^{(n)} \sigma_{2^{n+1}-2}^{(n)} \cdots \sigma_{j}^{(n)}(\gamma)\right) ;
$$

but, by Lemma 25, we must have $\alpha_{j}^{(2 n+3)}=\left(\alpha_{j}^{(2 n+2)}, a_{j}\right)$ for some $a_{j} \in\{1,2\}$; since $L_{1}^{(1)}=L_{2}^{(1)}=i d_{\Omega_{3}}$ and $L_{3}^{(1)}=(12)$, it follows from (iii) that

$$
\left(L_{2}^{(2 n+3)} L_{3}^{(2 n+3)}\right)^{2^{n+1}}\left(\alpha_{j}^{(2 n+3)}\right)=\left(\alpha_{j}^{(2 n+2)}, \tilde{a_{j}}\right)
$$

where $\tilde{a_{j}}$ is the 'flip' of $a_{j}\left(=(12)\left(a_{j}\right)\right)$.

It follows that $\left\{\alpha_{l}^{(2 n+3)}=\left(L_{2}^{(2 n+3)} L_{3}^{(2 n+3)}\right)^{l} \alpha_{0}^{(2 n+3)}: 0 \leq l<2^{n+2}\right\}$ is a set of $2^{n+2}$ distinct elements. Lemma 25 now implies that this set must be all of $X_{2 n+3}$; thus the cyclic group generated by $L_{2}^{(2 n+3)} L_{3}^{(2 n+3)}$ does indeed act transitively on $X_{2 n+3}$; since the order of that cyclic group (viewed as permutations of $X_{2 n+3}$ ) must necessarily equal the cardinality of $X_{2 n+3}$, we have proved (i) and (ii) of (a)'. 
The above proof also shows that $\left\{\alpha_{j}^{(2 n+4)}, \alpha_{j+2^{n+1}}^{(2 n+4)}\right\}=\left\{\alpha_{j}^{(2 n+2)}\right\} \times\{(1,3),(2,3)\}$ for $0 \leq j<2^{n+1}$. Let us write $C_{x, y}^{(l)}=\#\left\{j: 0 \leq j<2^{l+1}, \sigma_{j}^{(l)}=L_{x} L_{y}\right\}$ for $x, y \in \Omega_{3}, x \neq y$. The first statement of this paragraph, the definition of the $\sigma_{j}^{(l)}$ 's, and the last two tables occurring in this proof, show that $C_{1,3}^{(n+1)}=C_{3,1}^{(n)}+$ $C_{3,2}^{(n)}, C_{3,1}^{(n+1)}=C_{1,3}^{(n)}+C_{2,3}^{(n)}, C_{2,3}^{(n+1)}=C_{2,1}^{(n)}+C_{1,2}^{(n)}, C_{3,2}^{(n+1)}=C_{1,2}^{(n)}+C_{2,1}^{(n)}$.

Hence,

$$
\begin{aligned}
\sum_{j=0}^{2^{n+2}-1} w\left(\sigma_{j}^{(n+1)}\right) & =C_{1,3}^{(n+1)}+C_{3,1}^{(n+1)}+C_{2,3}^{(n+1)}+C_{3,2}^{(n+1)} \\
& =C_{3,1}^{(n)}+C_{3,2}^{(n)}+C_{1,3}^{(n)}+C_{2,3}^{(n)}+2\left(C_{1,2}^{(n)}+C_{2,1}^{(n)}\right) \\
& \equiv \sum_{j=0}^{2^{n+1}-1} w\left(\sigma_{j}^{(n)}\right)(\bmod 2),
\end{aligned}
$$

thus establishing (iii) of $(a)^{\prime}$.

This completes the inductive step and hence the proof of $(a)^{\prime}$, and, in particular, the proof of (a).

(b) The proof is by induction on $n$. When $n=0$, we have $X_{1}=\{1,2\}$, and $1 \not \psi_{1} 2$ since $\phi_{1}=\rho_{1}=i d \neq \rho_{2}=\phi_{2}$. Assume that the statement (b) is valid with $n$ replaced by $n-1$, and that $n>1$.

Suppose $X_{2 n+1}$ contains two distinct elements which are equivalent (with respect to $\sim_{2 n+1}$ ). Since $G_{2 n+1}$ acts transitively on $X_{2 n+1}$ and preserves equivalence, we may assume that $\alpha_{0}^{(2 n+1)} \sim_{2 n+1} \alpha_{j}^{(2 n+1)}$ for some $j$ with $0<j<2^{n+1}$; further, we may assume that $j$ is the smallest positive integer $l$ for which $\alpha_{l}^{(2 n+1)} \sim_{2 n+1} \alpha_{0}^{(2 n+1)}$. Since $L_{2} L_{3}$ preserves equivalence, this minimality assumption is seen to imply that $j \mid 2^{n+1}$; thus $j=2^{m}$ for some $m$.

Thus $\alpha_{0}^{(2 n+1)} \sim_{2 n+1} \alpha_{2^{m}}^{(2 n+1)}$, where $1 \leq m<n$. By (a)' (ii) with $m$ in place of $n$, we see that there exists $\gamma \in X_{2 n+1-(2 m+2)}$ such that $\alpha_{j}^{(2 n+1)}=\left(\alpha_{0}^{(2 m+2)}, \gamma\right)$. An appeal to the cancellation law - cf. Proposition 17(e) - and the induction hypothesis results in the desired contradiction, thus completing the proof of (b).

(c) Suppose $\alpha \in \Omega_{3}^{2 n+1}$ and $\alpha \sim_{2 n+1} \beta \in X_{2 n+1}$. We shall prove that $\alpha \in X_{2 n+1}$ and appeal to (b). Let $\tilde{\alpha} \in \Omega_{3}^{2 n+1}$ be a reduced element such that $\alpha \sim_{2 n+1} \tilde{\alpha}$ and $\tilde{\alpha}$ is constructed from $\alpha$ as in the proof of Lemma 23. Since $G_{2 n+1}$ preserves $\sim_{2 n+1}$, and since the $G_{2 n+1}$-orbit of $\beta$ contains $2^{n+1}$ elements which are pairwise inequivalent, it follows that the $G_{2 n+1}$-orbit of $\tilde{\alpha}$ contains at least $2^{n+1}$ elements. It follows easily now from Lemma 25 that $\tilde{\alpha} \in X_{2 n+1}$. The manner in which $\tilde{\alpha}$ was constructed from $\alpha$ in the proof of Lemma 23 shows that $\alpha$ must have been in $X_{2 n+1}$ to start with.

Corollary 27. Let $\alpha_{0}^{(2 n)}=(1,3,1,3, \cdots, 1,3)$ and $\beta_{0}^{(2 n)}=(3,1,3,1, \cdots 3,1)$. Let $X_{2 n}$ (resp. $Y_{2 n}$ ) denote the set of reduced elements of the same configuration as $\alpha_{0}^{(2 n)}$ (resp., $\beta_{0}^{(2 n)}$ ). Then $\alpha \sim_{2 n} \tilde{\alpha} \in X_{2 n} \Rightarrow \alpha=\tilde{\alpha}$ and $\beta \sim_{2 n} \tilde{\beta} \in Y_{2 n} \Rightarrow$ $\beta=\tilde{\beta}$.

Proof. Suppose $\alpha \sim_{2 n} \tilde{\alpha} \in X_{2 n}$. Then $(\alpha, 1) \sim_{2 n+1}(\tilde{\alpha}, 1) \in X_{2 n+1}$, and so Lemma 26 implies that $(\alpha, 1) \in X_{2 n+1}$, whence $\alpha \in X_{2 n}$ and $(\alpha, 1)=(\tilde{\alpha}, 1)$, and hence 
$\alpha=\tilde{\alpha}$. The proof of the second assertion is similar; just append a 1 at the beginning.

We are now ready to state the main fact about this example.

Theorem 28. The principal graph, in this case, is infinite.

Proof. Suppose a subfactor $N \subset M$ has finite principal graph $\mathcal{G}$; let the set of even (resp., odd) vertices be denoted by $\mathcal{G}^{(0)}$ (resp., $\left.\mathcal{G}^{(1)}\right)$.

If $N=M_{-1} \subset M=M_{0} \subset M_{1} \subset M_{2} \subset \cdots$ is the tower of the basic construction, then (see $[\mathrm{O}],[\mathrm{P}])$ there exist identifications $N^{\prime} \cap M_{2 k} \cong \bigoplus_{v \in \mathcal{G}^{(1)}} M_{n_{2 k+1}(v)}(\mathbb{C})$ and $N^{\prime} \cap M_{2 k-1} \cong \bigoplus_{v \in \mathcal{G}^{(0)}} M_{n_{2 k}(v)}(\mathbb{C})$, where $n_{l}(v)$ denotes the number of paths in $\mathcal{G}$ of length $l$ which start at $*$ in $\mathcal{G}^{(0)}$ and end in $v$. In particular, for any $v \in \mathcal{G}^{(1)}, \lim _{k \rightarrow \infty} n_{2 k+1}(v)=\infty$ (and for any $v \in \mathcal{G}^{(0)}, \lim _{k \rightarrow \infty} n_{2 k+1}(v)=\infty$ ).

In particular if $N \subset M$ is a subfactor such that for each $k \geq 1$ there exists a minimal central projection $p_{k}$ in $N^{\prime} \cap M_{k}$ such that $\left(N^{\prime} \cap M_{k}\right) p_{k} \cong \mathbb{C}$, then $N$ must be of infinite depth.

In view of Proposition 10, Lemma 26(c) and Corollary 27, the subfactor we are concerned with in Case 11 satisfies the property of the preceding paragraph, and the proof of the theorem is complete.

Without going through the computations, we just show what the subgraph of the principal graph induced by the set of vertices at distance at most 4 from $*$ looks like. (This amounts to computing the Bratteli diagrams for the tower $\left\{N^{\prime} \cap M_{k}\right\}_{-1 \leq k \leq 3}$.)

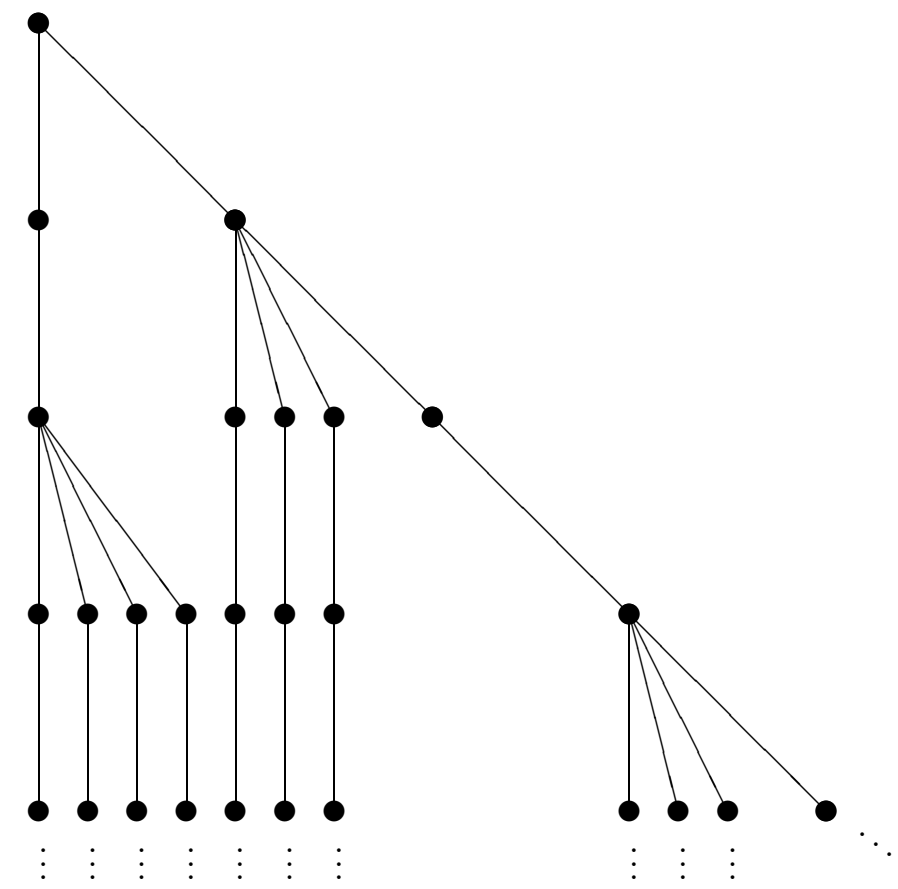

FIGURE 11 
It is our belief that the infinite principal graph is a tree, and we have a guess as to its description, but we shall say no more about this case.

Case $(15): \lambda=(i d,(12),(13))=\psi, \rho=(i d, i d,(12))=\phi$.

The analysis of this case will also be broken into a series of lemmas.

Lemma 29. (a) $\rho_{\alpha}(3)=3$ and $\rho_{\alpha}\{1,2\}=\{1,2\}$ for all $a \in \Omega_{3}^{k}, k \geq 1$;

(b) $L_{3}=(13) \times(13) \times(13) \times \cdots$.

Proof. (a) Clear.

(b) Since $\lambda_{\rho_{\alpha}^{-1}(3)}=\lambda_{3}=(13)$ for all $\alpha \in \Omega_{3}^{k}, k \geq 1$, and since

$$
\left(L_{3} \alpha\right)_{k}=\lambda_{\rho_{\left(\alpha_{1}, \cdots, \alpha_{k-1}\right)}^{-1}(3)}\left(\alpha_{k}\right)
$$

the conclusion follows.

Lemma 30. Let $\alpha \in \Omega_{3}^{\mathbb{N}}$ be written in the form $\alpha=\left(w_{1}, 3, w_{2}, 3, w_{3}, 3, \cdots\right)$ where each $w_{i}$ is a (possibly empty) sequence of 1 's and 2 's. Then

$$
L_{1}(\alpha)=\left(w_{1}, 3, \tilde{w}_{2}, 3, w_{3}, 3, \tilde{w}_{4}, 3, \cdots\right), L_{2}(\alpha)=\left(\tilde{w}_{1}, 3, w_{2}, 3, \tilde{w}_{3}, 3, w_{4}, 3, \cdots\right)
$$

where $\tilde{w}$ denotes the word obtained by changing every 1 in $w$ to 2 and changing every 2 in $w$ to 1 . In particular,

$$
L_{1} L_{2}=L_{2} L_{1}=(12) \times(12) \times(12) \times \cdots
$$

Proof. Since $\rho_{\alpha}\{1,2\}=\{1,2\}$ for all $\alpha \in \Omega_{3}^{k}, k \geq 1$, and since $\lambda_{x}(3)=3$ for $x=1,2$, it is clear that if $x \in\{1,2\}$ and $\alpha_{l}=3$, then also $\left(L_{x}(\alpha)\right)_{l}=3$.

Since $\rho_{\alpha}=(12)^{l_{3}(\alpha)}$, it follows that if $\left\{l: \alpha_{l}=3\right\}=\left\{n_{1}, n_{2}, \cdots\right\}$ where $n_{1}<n_{2}<\cdots$, then

$$
\rho_{\left(\alpha_{1}, \cdots, \alpha_{l}\right)}= \begin{cases}(12), & \text { if } n_{2 p+1} \leq l<n_{2 p+2}, p=0,1,2, \cdots, \\ i d_{\Omega_{3}}, & \text { if } n_{2 p} \leq l<n_{2 p+1}, p=0,1,2, \cdots,\end{cases}
$$

where we write $n_{0}=0$.

The conclusion of the lemma follows from the foregoing fact and equation (10).

Corollary 31. There exists an embedding $\chi: S_{3} \mapsto \tilde{G}$ such that $\chi((13))=L_{3}$ and $\chi((12))=L_{1} L_{2}$. Furthermore, $\tilde{G}=G$.

Proof. The first assertion is clear, in view of the preceding lemmas. For the second, note that by definition $G$ is the subgroup of $\tilde{G}$ consisting of elements of $G$ which are expressible as a product of an even number of $L_{x}$ 's. Notice, however, that $L_{1} L_{2} L_{3}=\chi((132))$ is an element of order 3 , and hence $\left(L_{1} L_{2} L_{3}\right)^{3}$ is a product of nine $L_{x}$ 's which is equal to the identity. Hence, for any $x$ in $\Omega_{3}$, we have $L_{x}=L_{x}\left(L_{1} L_{2} L_{3}\right)^{3} \in \tilde{G}$; hence $G=\tilde{G}$.

Before proceeding further, let us introduce the notation $X_{k}=\left\{\alpha \in \Omega_{3}^{k}: \alpha_{i} \neq\right.$ $\alpha_{i+1}$ for $\left.1 \leq i<k\right\}$. ( We shall not, while discussing Case 15, need the set denoted by $X_{k}$ used while discussing Case 11; so no confusion should arise.)

Recall Corollary $18(\mathrm{~b})$, which says that $X_{k}$ is stable under $G_{k}$. We now come to a crucial step in our analysis. 
Lemma 32. Fix an integer $k \geq 1$.

(a) $\# X_{k}=3 \cdot 2^{k-1}$.

(b) Let $\gamma_{0}^{(k)}$ denote the restriction to $X_{k}$ of $L_{1}^{(k)} L_{3}^{(k)}$, and let $\Gamma_{0}^{(k)}$ denote the cyclic subgroup generated by $\gamma_{0}^{(k)}$. Then,

(i) $\# \Gamma_{0}^{(k)}=2^{k}$;

(ii) $X_{k}$ splits into two orbits, $B_{j}^{(k)}, j=1,2$, under the action of $\Gamma_{0}^{(k)}$, where

$$
B_{1}^{(k)}=\left\{\alpha \in X_{k}: \alpha_{1} \in\{1,3\}\right\}
$$

and

$$
B_{2}^{(k)}=\left\{\alpha \in X_{k}: \alpha_{1}=2\right\}
$$

(c) $G_{k}$ acts transitively on $X_{k}$.

(d) $\alpha, \beta \in X_{k}$ and $\alpha \neq \beta \Rightarrow \alpha \varkappa_{k} \beta$.

Proof. (a) Trivial.

(b) As in the proof of Lemma 26, we start with a table, which has the same interpretation as the tables occurring in the proof of that lemma; here $\beta \in \Omega_{3}^{\mathbb{N}}$ :

\begin{tabular}{|c|c|c|c|c|c|}
\hline & $L_{1}$ & $L_{2}$ & $L_{3}$ & $L_{1} L_{3}$ & $L_{2} L_{3}$ \\
\hline$(1, \beta)$ & $\left(1, L_{1} \beta\right)$ & $\left(2, L_{2} \beta\right)$ & $\left(3, L_{3} \beta\right)$ & $\left(3, L_{2} L_{3} \beta\right)$ & $\left(3, L_{1} L_{3} \beta\right)$ \\
\hline$(2, \beta)$ & $\left(2, L_{1} \beta\right)$ & $\left(1, L_{2} \beta\right)$ & $\left(2, L_{3} \beta\right)$ & $\left(2, L_{1} L_{3} \beta\right)$ & $\left(1, L_{2} L_{3} \beta\right)$ \\
\hline$(3, \beta)$ & $\left(3, L_{2} \beta\right)$ & $\left(3, L_{1} \beta\right)$ & $\left(1, L_{3} \beta\right)$ & $\left(1, L_{1} L_{3} \beta\right)$ & $\left(2, L_{2} L_{3} \beta\right)$ \\
\hline
\end{tabular}

The last two columns of the table show that: if we write $S=\left\{L_{1} L_{3}, L_{2} L_{3}\right\}$, and if $\sigma \in S$ - say $\sigma=L_{x} L_{y}$, with $(x, y) \in\{(1,3),(2,3)\}$ - then for any $k \geq 1$ and any $\alpha \in \Omega_{3}^{k}$, there exists a uniquely defined $\sigma^{\prime} \in S$ such that

$$
\sigma(\alpha, \beta)=\left(L_{x}^{(k)} L_{y}^{(k)}(\alpha), \sigma^{\prime}(\beta)\right)
$$

for all $\beta \in \Omega_{3}^{\mathbb{N}}$. Set $w\left(L_{1} L_{3}\right)=1$ and $w\left(L_{2} L_{3}\right)=0$.

We shall need the above observation in the statement (and proof) of the following crucial assertion:

Assertion $(*)$ : Fix a positive integer $k$. Then there exists $\alpha_{0}^{(k)}$ in $X_{k}$ with first coordinate equal to 1, with the following properties: define $\alpha_{j}^{(k)}=\left(\gamma_{0}^{(k)}\right)^{j}\left(\alpha_{0}^{(k)}\right)$ for $1 \leq j \leq 2^{k}$, and let $\sigma_{j}^{(k)}$ be the uniquely defined element of $S$ such that $\left(L_{1} L_{3}\right)\left(\alpha_{j}^{(k)}, \beta\right)=\left(\alpha_{j+1}^{(k)}, \sigma_{j}^{(k)}(\beta)\right)$ for all $\beta \in \Omega_{3}^{\mathbb{N}}$ for $0 \leq j<2^{k}$. Then, the following statements are valid:

$$
\alpha_{j}^{(k)} \neq \alpha_{l}^{(k)} \quad \text { for } 0 \leq j<l<2^{k}
$$

$$
\alpha_{2^{k}}^{(k)}=\alpha_{0}^{(k)}
$$

$$
\sum_{j=0}^{2^{k}-1} w\left(\sigma_{j}^{(k)}\right) \text { is an odd integer. }
$$

We prove Assertion $(*)$ by induction on $k$. When $k=1$, we have $X_{1}=\{1,2,3\}$ and $\gamma_{0}^{(1)}=(13)$; put $\alpha_{0}^{(1)}=(1)$; then $\alpha_{1}^{(1)}=(3)$ and $\alpha_{2}^{(1)}=(1)$; the preceding 
table shows that $\sigma_{0}^{(1)}=L_{2} L_{3}$ and $\sigma_{1}^{(1)}=L_{1} L_{3}$, whence $w\left(\sigma_{0}^{(1)}\right)+w\left(\sigma_{1}^{(1)}\right)=0+$ $1=1$.

Suppose Assertion $(*)$ is valid for some integer $k$. Note that for any $\beta$ in $\Omega_{3}^{\mathbb{N}}$, we have, by the induction hypothesis,

$$
\left(L_{1} L_{3}\right)^{2^{k}}\left(\alpha_{0}^{(k)}, \beta\right)=\left(\alpha_{0}^{(k)}, \sigma_{2^{k}-1}^{(k)} \sigma_{2^{k}-2}^{(k)} \cdots \sigma_{0}^{(k)}(\beta)\right) .
$$

Observe that $L_{1}^{(1)} L_{3}^{(1)}=(13)$, while $L_{2}^{(1)} L_{3}^{(1)}=$ (132); hence any product $\pi_{m} \cdots \pi_{0}$, where each $\pi_{j}$ is either $L_{2}^{(1)} L_{3}^{(1)}$ or $L_{1}^{(1)} L_{3}^{(1)}$, is an even or odd permutation according as $w$ is even or odd, where $w=\#\left\{i: 0 \leq i \leq m, w_{i}=L_{1}^{(1)} L_{3}^{(1)}\right\}$. Let $\pi_{j} \in S_{3}, 0 \leq j<2^{k}$, be defined by $\pi_{j}=L_{x}^{(1)} L_{y}^{(1)}$ if $\sigma_{j}^{(k)}=L_{x} L_{y}$. Then, by the statement ( $*$ iii), it follows that $\pi=\pi_{2^{k}-1} \pi_{2^{k}-2} \cdots \pi_{0}$ is an odd permutation in $S_{3}$; i.e., $\pi$ is a transposition. Let $a_{0} \in\{1,2,3\}$ be such that $a_{0}$ is moved by $\pi$, and such that $a_{0}$ is distinct from the last coordinate of $\alpha_{0}^{(k)}$. Put $\alpha_{0}^{(k+1)}=\left(\alpha_{0}^{(k)}, a_{0}\right)$. Then $\alpha_{0}^{(k+1)} \in X_{k+1}$, the first co-ordinate of $\alpha_{0}^{(k+1)}$ is 1 , and, more importantly,

$$
\left(L_{1}^{(k+1)} L_{3}^{(k+1)}\right)^{2^{k}}\left(\alpha_{0}^{(k+1)}\right)=\left(\alpha_{0}^{(k)}, \tilde{a_{0}}\right)
$$

where $\tilde{a_{0}}=\pi\left(a_{0}\right)$; hence $\left(\gamma_{0}^{(k+1)}\right)^{2^{k}}\left(\alpha_{0}^{(k+1)}\right) \neq \alpha_{0}^{(k+1)}$.

For this choice of $\alpha_{0}^{(k+1)}$, let $\alpha_{j}^{(k+1)}, 1 \leq j \leq 2^{k+1}$, and $\sigma_{j}^{(k+1)}, 0 \leq j \leq 2^{k+1}$, be defined as in Assertion(*). It must be clear that, for $0 \leq j<2^{k}, \alpha_{j}^{(k+1)}=\left(\alpha_{j}^{(k)}, a_{j}\right)$ for some $a_{j} \in \Omega_{3}$; by choice, we have $\alpha_{2^{k}}^{(k+1)}=\left(\alpha_{0}^{(k)}, \tilde{a_{0}}\right)$ with $\tilde{a_{0}} \neq a_{0}$. It follows easily that $\alpha_{2^{k}+j}^{(k+1)}=\left(\alpha_{j}^{(k)}, \tilde{a_{j}}\right)$ for some $\tilde{a_{j}} \in \Omega_{3}, 0 \leq j<2^{k}$; since $\alpha_{0}^{(k+1)} \neq \alpha_{2^{k}}^{(k+1)}$ and since $\gamma_{0}^{(k+1)}$ is injective, it follows that $a_{j} \neq \tilde{a_{j}}$ for $0 \leq j<2^{k}$. Thus, we have proved $(* \mathrm{i})$ for $k+1$.

As for $\left(*\right.$ ii), since $\left(L_{1}^{(k)} L_{3}^{(k)}\right)\left(\alpha_{2^{k}-1}^{(k)}\right)=\alpha_{0}^{(k)}$, it must be the case that

$$
\left(L_{1}^{(k+1)} L_{3}^{(k+1)}\right)\left(\alpha_{2^{k+1}-1}^{(k+1)}\right)=\left(L_{1}^{(k+1)} L_{3}^{(k+1)}\right)\left(\alpha_{2^{k}-1}^{(k)}, \tilde{a}_{2^{k}-1}\right)=\left(\alpha_{0}^{(k)}, b\right)
$$

for some $b$ in $\Omega_{3}$. But since $X_{k+1}$ is stable under $G_{k}$, we know that $a$ and $\tilde{a}$ are the only two elements of $\Omega_{3}$ such that $\left(\alpha_{0}^{(k)}, a\right),\left(\alpha_{0}^{(k)}, \tilde{a}\right) \in X_{k+1}$. Hence $b \in\{a, \tilde{a}\}$. Since $\gamma_{0}^{(k+1)}$ is injective and $\gamma_{0}^{(k+1)}\left(\alpha_{2^{k}-1}^{(k+1)}\right)=\left(\alpha_{0}^{(k)}, \tilde{a}\right)$, it must be that $b=a$; i.e., $\alpha_{2^{k+1}}^{(k+1)}=\alpha_{0}^{(k+1)}$, as desired.

Now for $\left(*\right.$ iii). For $l=k, k+1, x \in \Omega_{3}$ and $\sigma \in S\left(=\left\{L_{1} L_{3}, L_{2} L_{3}\right\}\right)$, define

$$
C_{x, \sigma}^{(l)}=\#\left\{j: 0 \leq j<2^{l},\left(\alpha_{j}^{(l)}\right)_{l}=x, \sigma_{j}^{(l)}=\sigma\right\}
$$

(where, of course, $\left(\alpha_{j}^{(l)}\right)_{l}$ denotes the $l$-th coordinate of $\left.\alpha_{j}^{(l)}\right)$.

To make the exposition clear, suppose some $\alpha_{j}^{(k)}$ ends in 2 and $\sigma_{j}^{(k)}=L_{1} L_{3}$. Then, the proof of $(* \mathrm{i})$ shows that $\left\{\alpha_{j}^{(k+1)}, \alpha_{2^{k}+j}^{(k+1)}\right\}=\left\{\alpha_{j}^{(k)}\right\} \times\{1,3\}$. The column indexed by $L_{1} L_{3}$ and the rows indexed by $(1, \beta)$ and $(3, \beta)$ in the table at the start of this proof show then that $\left\{\sigma_{j}^{(k+1)}, \sigma_{2^{k}+j}^{(k+1)}\right\}=\left\{L_{2} L_{3}, L_{1} L_{3}\right\}$.

More generally, consider the bipartite graph $\Lambda$ with the sets $\Lambda^{(0)}$ and $\Lambda^{(1)}$ of even and odd vertices both being indexed by $\Omega_{3} \times S$ : if $(x, \sigma)_{0}$ is an even vertex in $\Lambda$, let $\Omega_{3} \backslash\{x\}=\{y, z\}$; if $\sigma(y, \beta)=\left(\tilde{y}, \sigma_{1}(\beta)\right)$ and $\sigma(z, \beta)=\left(\tilde{z}, \sigma_{2}(\beta)\right)$, for arbitrary $\beta \in \Omega_{3}^{\mathbb{N}}$, and appropriate $\sigma_{1}, \sigma_{2} \in S$, then $(x, \sigma)_{0}$ is joined, in the graph $\Lambda$, to the odd vertices $\left(y, \sigma_{1}\right)_{1}$ and $\left(z, \sigma_{2}\right)_{1}$. Thus the graph is 


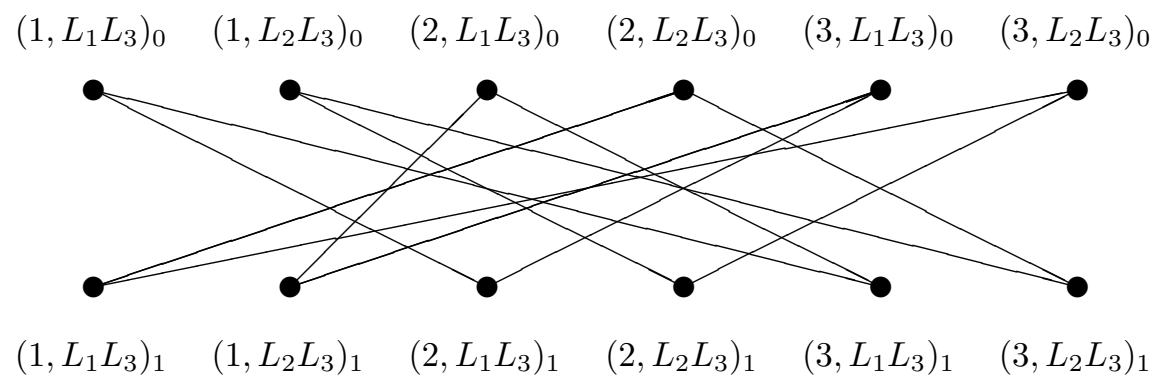

Figure B

A little thought and an inspection of the above graph shows that

$$
\begin{array}{ll}
C_{1, L_{1} L_{3}}^{(k+1)}=C_{2, L_{2} L_{3}}^{(k)}+C_{3, L_{2} L_{3}}^{(k)}, & C_{1, L_{2} L_{3}}^{(k+1)}=C_{2, L_{1} L_{3}}^{(k)}+C_{3, L_{1} L_{3}}^{(k)}, \\
C_{2, L_{1} L_{3}}^{(k+1)}=C_{1, L_{1} L_{3}}^{(k)}+C_{3, L_{1} L_{3}}^{(k)}, & C_{2, L_{2} L_{3}}^{(k+1)}=C_{1, L_{2} L_{3}}^{(k)}+C_{3, L_{2} L_{3}}^{(k)}, \\
C_{3, L_{1} L_{3}}^{(k+1)}=C_{1, L_{1} L_{3}}^{(k)}+C_{2, L_{1} L_{3}}^{(k)}, & C_{3, L_{2} L_{3}}^{(k+1)}=C_{1, L_{2} L_{3}}^{(k)}+C_{2, L_{2} L_{3}}^{(k)} .
\end{array}
$$

These relations hold for all $k$.

Thus, the $C_{x, \sigma}^{(k)}$ 's are defined by the above recursion relations, and the initial conditions $C_{1, L_{2} L_{3}}^{(1)}=C_{3, L_{1} L_{3}}^{(1)}=1, C_{x, \sigma}^{(1)}=0$ otherwise. We claim that for all $k \geq 1$ the number $C_{1, L_{1} L_{3}}^{(k)}+C_{2, L_{2} L_{3}}^{(k)}+C_{3, L_{2} L_{3}}^{(k)}$ is even, and $C_{1, L_{1} L_{3}}^{(k)}+C_{2, L_{1} L_{3}}^{(k)}+C_{3, L_{1} L_{3}}^{(k)}$ is odd.

These are true for $k=1$; if they are known to be true for $k$, then the foregoing recursion relations show that

$$
\begin{aligned}
C_{1, L_{1} L_{3}}^{(k+1)}+C_{2, L_{2} L_{3}}^{(k+1)}+C_{3, L_{2} L_{3}}^{(k+1)} & =2\left(C_{1, L_{2} L_{3}}^{(k)}+C_{2, L_{2} L_{3}}^{(k)}+C_{3, L_{2} L_{3}}^{(k)}\right) \\
& =\text { even integer }
\end{aligned}
$$

and

$$
\begin{aligned}
& C_{1, L_{1} L_{3}}^{(k+1)}+C_{2, L_{1} L_{3}}^{(k+1)}+C_{3, L_{1} L_{3}}^{(k+1)} \\
& \quad=\left(C_{1, L_{1} L_{3}}^{(k)}+C_{2, L_{1} L_{3}}^{(k)}+C_{3, L_{1} L_{3}}^{(k)}\right)+\left(C_{1, L_{1} L_{3}}^{(k)}+C_{2, L_{2} L_{3}}^{(k)}+C_{3, L_{2} L_{3}}^{(k)}\right) \\
& \quad=\quad \text { odd integer (by the induction hypothesis). }
\end{aligned}
$$

In particular we have proved that

$$
\sum_{j=0}^{2^{k+1}-1} w\left(\sigma_{j}^{(k+1)}\right) \equiv C_{1, L_{1} L_{3}}^{(k+1)}+C_{2, L_{1} L_{3}}^{(k+1)}+C_{3, L_{1} L_{3}}^{(k+1)}(\bmod 2),
$$

which is odd, and the proof of Assertion(*) is complete.

To proceed with the proof of the lemma, notice from our table that $\left(L_{1} L_{3}\right)(2, \beta)$ $=\left(2, L_{1} L_{3} \beta\right)$ for all $\beta$ in $\Omega_{3}^{\mathbb{N}}$. Hence, in the notation of the statement (b) in the lemma, the group $\Gamma_{0}^{(k)}$ leaves $B_{2}^{(k)}$ (and hence $B_{1}^{(k)}=X_{k} \backslash B_{2}^{(k)}$ ) invariant. Since $\# B_{1}^{(k)}=2^{k}$, it follows from Assertion $(*)$ that $\Gamma_{0}^{(k)}$ acts transitively on $B_{1}^{(k)}$ for each $k$. 
However, $B_{2}^{(k)}=\{2\} \times B_{1}^{(k-1)}$, and since $\gamma_{0}^{(k)}$ acts on $B_{2}^{(k)}=\{2\} \times B_{1}^{(k-1)}$ as $i d \times \gamma_{1}^{(k-1)}$, the preceding conclusion about $B_{1}^{(k)}$, for each $k$, now shows that $\left.\gamma_{0}^{(k)}\right|_{B_{2}^{(k)}}$ has order $2^{k-1}$. This completes the proof of (b).

(c) Notice that, by Lemma $30,\left(L_{1}^{(k)} L_{2}^{(k)}\right)\left(\begin{array}{lllll}1 & 3 & 1 & 3 & \cdots\end{array}\right)=\left(\begin{array}{lllll}2 & 3 & 2 & 3 & \cdots\end{array}\right)$; this observation, together with (b), completes the proof of (c).

(d) Since the cyclic group $\Gamma_{0}^{(k)}$ acts transitively on each of $B_{1}^{(k)}$ and $B_{2}^{(k)}$, we can repeat the argument given to prove Lemma 26 (b) to see that for $j=1,2$, no two distinct elements of $B_{j}^{(k)}$ are equivalent (with respect to $\sim_{k}$ ). Since $\Gamma_{0}^{(k)}$ preserves equivalence, since $B_{1}^{(k)}$ (resp., $B_{2}^{(k)}$ ) consists of $2^{k}$ (resp., $2^{k-1}$ ) inequivalent elements, no element of $B_{1}^{(k)}$ can be equivalent to $B_{2}^{(k)}$. (The argument for this is exactly like the proof of Lemma 26 (c).) The proof of the lemma is finally complete.

In the sequel, if $a \in \Omega_{3}$, we shall find it convenient to denote by $a^{(k)}$ the constant sequence in $\Omega_{3}^{k}$ with all coordinates equal to $a$. In the proof of the next lemma, we shall need the obvious consequence of Proposition 17(d) that $\left(\alpha, a^{(m)}\right) \sim\left(\alpha, b^{(m)}\right)$ for any $\alpha \in \Omega_{3}^{(l)}, l \geq 1$, and any $a, b \in \Omega_{3}$, provided $m$ is even.

Lemma 33. Let $k \geq 1$ and $\beta \in \Omega_{3}^{k}$. Then there exist a unique integer $l \leq k$ and an element $\alpha \in X_{l}$ such that:

(i) $\beta \sim_{k}\left(\alpha, \alpha_{l}^{(k-l)}\right)$; and

(ii) $0 \leq l \leq k$ and $l \equiv k(\bmod 2)$, where the possibility $l=0$ is interpreted to mean that $k$ is even and $\beta \sim_{k} 1^{(k)} \sim_{k} 2^{(k)} \sim_{k} 3^{(k)}$.

Proof. Existence. It follows easily from Lemma 20 and Proposition 17 (d) that there exist an $l \leq k$ and $\alpha \in X_{l}$ such that $\beta \sim_{k}\left(\alpha, \alpha_{l}^{(k-l)}\right)$. If this $l$ were such that $l \equiv k(\bmod 2)$, we are done; if $(k-l)$ is odd, and $l=0$, then $\beta \sim_{k} 1^{(k)}$ with $k$ odd, and we may just as well choose $l=1$ and $\alpha=(1)$; if $(k-l)$ is odd and $l \geq 1$, then

$$
\beta \sim_{k}\left(\alpha_{1}, \cdots \alpha_{l}, \alpha_{l}^{(k-l)}\right)=\left(\alpha_{1}, \cdots \alpha_{l-1}, \alpha_{l}^{(k-l+1)}\right) \sim_{k}\left(\alpha_{1}, \cdots \alpha_{l-1}, \alpha_{l-1}^{(k-l+1)}\right),
$$

and the proof of existence is complete.

Uniqueness. Suppose $\beta \sim_{k} \beta^{\prime}=\left(\alpha, \alpha_{l}^{(k-l)}\right)$, with $l, \alpha$ as in the statement of the lemma. Corollary 18(ii) implies that $\left\{\pi\left(\beta^{\prime}\right): \pi \in G_{k}\right\} \subseteq\left\{\left(\gamma, \gamma_{l}^{(k-l)}: \gamma \in X_{l}\right\}\right.$; however, Lemma 32 (c) and Corollary 18 (ii) imply that the above inclusion is actually an equality.

Since $(k-l)$ is even, observe that, in view of Proposition 17 and Lemma 32 (d), for $\gamma, \kappa \in X_{l}$, and $m$ even, we have:

$$
\begin{aligned}
\left(\gamma, \gamma_{l}^{(m)}\right) \sim_{l+m}\left(\kappa, \kappa_{l}^{(m)}\right) & \Rightarrow\left(\gamma, \gamma_{l}^{(m)}\right) \sim\left(\kappa, \gamma_{l}^{(m)}\right) \\
& \Rightarrow \gamma=\kappa .
\end{aligned}
$$

Suppose $\beta \sim_{k} \beta^{\prime}=\left(\alpha, \alpha_{l}^{(k-l)}\right)$ as above.

Case (i) $: k$ is even and $l=0$. Then $\beta \sim_{k} 1^{(k)} \sim_{k} 2^{(k)} \sim_{k} 3^{(k)}$. Since $\left\{1^{(k)}, 2^{(k)}, 3^{(k)}\right\}$ is a $G_{k^{-}}$orbit, and since $G_{k}$ preserves $\sim_{k}$, we find that all elements in the $G_{k}$-orbit of $\beta$ must be equivalent; 
Case (ii) $: l \neq 0$. Then the $G_{k}$-orbit of $\beta^{\prime}$ consists of precisely $3 \cdot 2^{l-1}$ elements, which are pairwise inequivalent. Hence, the $G_{k}$-orbit of $\beta$ meets precisely $3 \cdot 2^{l-1}$ equivalence classes.

In any case, we see that if $\beta, \beta^{\prime}, l$ and $\alpha$ are as above, the $G_{k}$-orbit of $\beta$ meets precisely $n_{l}$ distinct equivalence classes, where

$$
n_{l}= \begin{cases}1, & \text { if } l=0, \\ 3 \cdot 2^{l-1}, & \text { if } l \geq 1\end{cases}
$$

Since $l \mapsto n_{l}$ is clearly an injective function, we see that $\beta$ determines $l$ uniquely. The proof is now complete in view of equation (12).

If $k$ is a positive integer, then the number of integers $l$ satisfying $0 \leq l \leq k$ and $l \equiv k(\bmod 2)$ is given by $\{k / 2\}$ where $\{x\}$ is the smallest integer which is strictly greater than $x$. (Thus $\left\{\frac{2 n+1}{2}\right\}=\left\{\frac{2 n}{2}\right\}=n+1$.)

Proposition 34. Let $k$ be a positive integer, and let $I_{k}=\{l: 0 \leq l \leq k, l \equiv$ $k(\bmod 2)\}$. For each $l$ in $I_{k}$ and $\alpha \in X_{l}$, define $E_{l, \alpha}^{(k)}=\left[\left(\alpha, \alpha_{l}^{(k-l)}\right)\right]_{\sim_{k}}=\{\beta \in$ $\left.\Omega_{3}^{k}: \beta \sim_{k}\left(\alpha, \alpha_{l}^{(k-l)}\right)\right\}$ ( with the obvious convention that if $k$ is even, then $\left.E_{0, \cdot}^{(k)}=\left\{\beta \in \Omega_{3}^{k}: \beta \sim_{k} 1^{(k)}\right\}\right)$. Then,

(a) $\left\{E_{l, \alpha}^{(k)}: l \in I_{k}, \alpha \in X_{l}\right\}$ is the partition of $\Omega_{3}^{k}$ into $\sim_{k}$-equivalence classes.

(b) For each fixed $l$ in $I_{k}$, the group $G_{k}$ acts transitively on the collection $O_{l}^{(k)}=$ $\left\{E_{l, \alpha}^{(k)}: \alpha \in X_{l}\right\}$ of equivalence classes, and hence $\left\{O_{l}^{(k)}: l \in I_{k}\right\}$ is the collection of $G_{k}$ - orbits of equivalence classes.

(c) $\left\{\sigma \in G_{k}: \sigma\left(E_{l, \alpha}^{(k)}\right)=E_{l, \alpha}^{(k)}\right\}=\left\{\sigma \in G_{k}: \sigma(\alpha)=\alpha\right\}$ for each $l \in I_{k}$ and $\alpha \in X_{l}$.

Proof. (a) is just a reformulation of Lemma 33.

(b) For fixed $l$ in $I_{k}$, let $R_{l}=\left\{\left(\alpha, \alpha_{l}^{(k-l)}\right): \alpha \in X_{l}\right\}$. It follows from Corollary 18 and Lemma 32 (c) that $G_{k}$ acts transitively on $R_{l}$. This proves (b).

(c) Notice that $E_{l, \alpha}^{(k)} \cap R_{l}=\left\{\left(\alpha, \alpha_{l}^{(k-l)}\right)\right\}$, thanks to Lemma 33. Since $R_{l}$ is stable under $G_{k}$, (c) is proved.

Definition 35. Fix a positive integer $k$. Define the following objects:

(a) $\pi_{k}: G \mapsto S\left(\Omega_{3}^{k}\right)$ is the unique homomorphism such that $\pi_{k}\left(L_{x}\right)=L_{x}^{(k)}$ for all $x$ in $\Omega_{3}^{k}$;

(b) $\alpha^{(k)}=(1,3,1,3, \cdots) \in \Omega_{3}^{k}$;

(c) $H_{k}=\left\{\sigma \in G: \pi_{k}(\sigma)\left(\alpha^{(k)}\right)=\alpha^{(k)}\right\}$; we shall find it convenient to also write $H_{0}=G$;

(d) for $0 \leq l \leq k, l \equiv k(\bmod 2), \pi_{l}^{(k)}: H_{l} \mapsto S\left(E_{l, \alpha^{(l)}}^{(k)}\right)$ is given by $\pi_{l}^{(k)}(\sigma)=$ $\pi_{k}(\sigma)_{E_{E_{l, \alpha}^{(l)}(l)}}$.

Recall that, by Proposition 34(c), the set $E_{l, \alpha(l)}^{(k)}$ is stable under $H_{l}$.

Notice that $G$ acts transitively on $X_{k}$ ( via $\sigma \mapsto \pi_{k}(\sigma)_{\left.\right|_{X_{k}}}$ ) and that the isotropy subgroup of the point $\alpha^{(k)}$ is precisely $H_{k}$; hence $\left[G: H_{k}\right]=3 \cdot 2^{k-1}$ for $k \geq 1$. Also, clearly, $H_{k} \supseteq H_{k+1}$. Thus we see that $\left\{H_{k}\right\}_{k=1}^{\infty}$ is a decreasing sequence of subgroups of $G$ such that for $k \geq 0$ we have

$$
\left[H_{k}: H_{k+1}\right]= \begin{cases}3, & \text { if } k=0 \\ 2, & \text { if } k \geq 1\end{cases}
$$


Also, in view of Proposition 10 and Proposition 34, we may deduce that the $k$-th relative commutant is given in this case by

$$
C_{k} \cong \bigoplus_{l \in I_{k}} \pi_{l}^{(k)}\left(H_{l}\right)^{\prime}
$$

(where we view $\pi_{l}^{(k)}$ as a unitary (permutation) representation in the natural manner).

In order to describe the inclusion of $C_{k}$ into $C_{k+1}$ ( via $x \mapsto x \otimes 1$ ), we need the next result.

Lemma 36. With the preceding notation, we have, for $k \geq 1$ and $l \in I_{k}$,

$$
\pi_{l}^{(k)}=\operatorname{Res}_{H_{l-1} \downarrow H_{l}} \pi_{l-1}^{(k-1)} \oplus \operatorname{Ind}_{H_{l+1} \uparrow H_{l}} \pi_{l+1}^{(k-1)},
$$

where, of course, Res and Ind denote restriction and induction, and we adopt the convention that the first (resp., second) summand above is non-existent when $l=0$ ( resp., $k=l$ ).

Proof. In the following, if $\alpha \in \Omega_{3}^{k}$, we write $[\alpha]_{k}=\left\{\alpha^{\prime} \in \Omega_{3}^{k}: \alpha \sim_{k} \alpha^{\prime}\right\}$.

Begin by observing the following consequence of the cancellation law (cf. Proposition $17(\mathrm{e})):$ if $\beta \in \Omega_{3}^{k+1}$ and $x \in \Omega_{3}$, then either $[\beta]_{k+1} \cap\left(\Omega_{3}^{k} \times\{x\}\right)=\emptyset$ or there exists an $\alpha$ in $\Omega_{3}^{k}$ such that $[\beta]_{k+1} \cap\left(\Omega_{3}^{k} \times\{x\}\right)=[\alpha]_{k} \times\{x\}$. Thus, for instance, if $k \geq 2$ and if $k$ is even, then

$$
E_{0, \cdot}^{(k)}=\coprod_{a \in \Omega_{3}} E_{1,(a)}^{(k-1)} \times\{a\} .
$$

Next, suppose $k \geq 2, l \in I_{k}$ and $0<l<k$; suppose $\beta=(\alpha, a) \in X_{l}$, - where $\alpha \in X_{l-1}$ and $a \in \Omega \backslash\left\{\alpha_{l-1}\right\}$. Then observe that

$$
\left(\alpha, a, a^{(k-l)}\right)=\left(\alpha, a^{(k-l)}, a\right) \sim\left(\alpha, \alpha_{l-1}^{(k-l)}, a\right) ;
$$

similarly, for any $b \in \Omega_{3} \backslash\{a\}$, observe that

$$
\left(\alpha, a, a^{(k-l)}\right) \sim\left(\alpha, a, b^{(k-l)}\right)=\left(\alpha, a, b, b^{(k-l-1)}\right) .
$$

Deduce that

$$
E_{l,(\alpha, a)}^{(k)}=\left(E_{l-1, \alpha}^{(k-1)} \times\{a\}\right) \amalg \bigcup_{b \in \Omega_{3} \backslash\{a\}}\left(E_{l+1,(\alpha, a, b)}^{(k-1)} \times\{b\}\right) .
$$

In view of Lemma 33 we also have, for $\beta=(\alpha, a) \in X_{k}, \alpha \in X_{k-1}, a \in \Omega_{3}$,

$$
E_{k,(\alpha, a)}^{(k)}=E_{k-1, \alpha}^{(k-1)} \times\{a\} .
$$

Recall our notation $\alpha^{(l)}=(1,3,1,3, \cdots) \in X_{l}$. Let us also simply write $E_{l}^{(k)}$ for $E_{l, \alpha^{(l)}}^{(k)}$. The preceding equations now imply that

$$
E_{l}^{(k)}=\left(E_{l-1}^{(k-1)} \times\left\{a_{l}\right\}\right) \amalg \bigcup_{b \in \Omega_{3} \backslash\left\{a_{l}\right\}}\left(E_{l+1,\left(\alpha^{(l)}, b\right)}^{(k-1)} \times\{b\}\right)
$$

where $a_{l}$ is 1 or 3 according as $l$ ( and hence $k$ ) is odd or even, with the understanding that if $l=0$ then the first term on the right side does not feature and the second term is interpreted as $\bigcup_{b \in \Omega_{3}}\left(E_{1,(b)}^{(k-1)} \times\{b\}\right)$, and if $l=k$, only the first term survives.

We prove the lemma by considering several cases (to account for the 'boundary' terms). 
Case (i) $: k=l$. Note, by definition, that $\pi_{k}^{(k)}$ is the trivial ( 1- dimensional) representation of $H_{k}$ ( since $E_{k}^{(k)}$ is a singleton, by Lemma 33). The conclusion of the lemma is trivially valid, since the restriction of the trivial representation is the trivial representation.

Case (ii) : $0<l<k$. Notice first that if $\alpha, \alpha^{\prime} \in \Omega_{3}^{k}$, and $a, b, x \in \Omega_{3}$, if $\alpha \sim_{k}$ $\alpha^{\prime}$ and $L_{x}^{(k+1)}(\alpha, a)=\left(L_{x}^{(k)}(\alpha), b\right)$, then $L_{x}^{(k+1)}\left(\alpha^{\prime}, a\right)=\left(L_{x}^{(k)}\left(\alpha^{\prime}\right), b\right)$ and $L_{x}^{(k)}(\alpha) \sim_{k} L_{x}^{(k)}\left(\alpha^{\prime}\right)$; hence if $\sigma \in G$ is arbitrary and $\pi_{k+1}(\sigma)(\alpha, a)=\left(\pi_{k}(\sigma)(\alpha), b\right)$, then $\pi_{k+1}(\sigma)\left(\alpha^{\prime}, a\right)=\left(\pi_{k}(\sigma) \alpha^{\prime}, b\right)$ and $\pi_{k}(\sigma)(\alpha) \sim_{k} \pi_{k}(\sigma)\left(\alpha^{\prime}\right)$. In particular, if $\pi_{k+1}(\sigma)(\alpha, a)=(\alpha, a)$, then $\pi_{k+1}(\sigma)$ maps $[\alpha]_{k} \times\{a\}$ into itself.

With $a_{l}$ as in equation (13), note that $\Omega_{3} \backslash\left\{a_{l}\right\}=\left\{a_{l+1}, 2\right\}$. By definition, the representation $\pi_{l}^{(k)}$ of $H_{l}$ acts on a vector space $V^{(l)}$ with basis indexed by $E_{l}^{(k)}$. Let $V_{1}^{(l)}$ (resp., $W_{1}^{(l)}$, resp., $W_{2}^{(l)}$ ) be the subspace of $V$ spanned by basis vectors indexed by $E_{l-1}^{(k-1)} \times\left\{a_{l}\right\}$ (resp., $E_{l+1,\left(\alpha^{(l)}, a_{l+1}\right)}^{(k-1)} \times\left\{a_{l+1}\right\}$, resp., $\left.E_{l+1,\left(\alpha^{(l)}, 2\right)}^{(k-1)} \times\{2\}\right)$. The preceding paragraph shows that $V_{1}^{(l)}$ is an invariant subspace for $\pi_{l}^{(k)}$ (and consequently, so is $\left.W_{1}^{(l)} \oplus W_{2}^{(l)}\right)$. In fact, we can even deduce from the previous paragraph that the subrepresentation of $\pi_{l}^{(k)}$ given by $V_{1}^{(l)}$ can be identified with $\operatorname{Res}_{H_{l-1} \downarrow H_{l}}\left(\pi_{l-1}^{(k-1)}\right)$.

Notice next that $\left[H_{l}: H_{l+1}\right]=2$; in fact, it follows from Lemma $32(\mathrm{~b})$ that $\left(L_{1} L_{3}\right)^{2^{l}} \in H_{l} \backslash H_{l+1}$ and that necessarily $\left(L_{1}^{(l+1)} L_{3}^{(l+1)}\right)^{2^{l}}\left(\alpha^{(l)}, 2\right)=\left(\alpha^{(l)}, a_{l+1}\right)$. Hence $\pi_{l}^{(k)}\left(\left(L_{1} L_{3}\right)^{2^{l}}\right)\left(W_{1}^{(l)}\right)=W_{2}^{(l)}$. On the other hand, it is clear that $\pi_{l}^{(k)}\left(H_{l+1}\right)$ leaves $W_{1}^{(l)}$ invariant and that the associated representation of $H_{l+1}$ is identifiable with $\pi_{l+1}^{k-1}$. Since, clearly, $\pi_{l}^{(k)}\left(\left(L_{1} L_{3}\right)^{2^{l}} H_{l+1}\right)\left(W_{1}^{(l)}\right)=W_{2}^{(l)}$, it follows easily that the subrepresentation of $\pi_{l}^{(k)}$ afforded by the invariant subspace $W_{1}^{(l)} \oplus W_{2}^{(l)}$ is identifiable with $\operatorname{Ind}_{H_{l+1} \uparrow H_{l}}\left(\pi_{l+1}^{(k-1)}\right)$.

Case (iii) $: l=0$ (and $k$ is even). In this case, as has already been noticed, $E_{0, .}^{(k)}=\left(E_{1,(1)}^{(k-1)} \times\{1\}\right) \amalg\left(E_{1,(2)}^{(k-1)} \times\{2\}\right) \amalg\left(E_{1,(3)}^{(k-1)} \times\{3\}\right)$. The representation $\pi_{0}^{(k)}$ of $H_{0}(=G)$ acts on a vector space $V_{0}^{(k)}$ with basis indexed by $\coprod_{i=1}^{3}\left(E_{1,(i)}^{(k-1)} \times\{i\}\right)$. Let $W_{i}^{(k-1)}$ be the subspace spanned by basis vectors indexed by $\left(E_{1,(i)}^{(k-1)} \times\{i\}\right)$, for $i=1,2,3$. The definitions show that $H_{0}=\coprod_{i=1}^{3} L_{i} H_{1}$ is the decomposition of $H_{0}$ into $H_{1}$-cosets (since $\pi_{1}\left(L_{1}\right)=i d_{\Omega_{3}}, \pi_{1}\left(L_{2}\right)=(12)$ and $\pi_{1}\left(L_{3}\right)=(13)$ ). For $i=1,2,3$, note that $\pi_{0}^{(k)}\left(L_{i}\right)\left(1^{(k)}\right)=i^{(k)}$, from which it follows that $\pi_{0}^{(k)}\left(L_{i}\right)\left(W_{1}^{(k-1)}\right)=W_{i}^{(k-1)}$. Also it is clear that $W_{1}^{(k-1)}$ is invariant under $\pi_{0}^{(k)}\left(H_{1}\right)$ ( since $\left.\pi_{k}\left(H_{1}\right)\left(1^{(k)}\right)=1^{(k)}\right)$, and that the subrepresentation of $H_{1}$ afforded by $W_{1}^{(k-1)}$ is seen to be identifiable with $\pi_{1}^{(k-1)}$. It follows that we may identify $\pi_{0}^{(k)}$ with $\operatorname{Ind}_{H_{1} \uparrow H_{0}}\left(\pi_{1}^{(k-1)}\right)$.

All the pieces are now in place for our final result.

Theorem 37. Let $H_{0} \supseteq H_{1} \supseteq H_{2} \supseteq \cdots$ be the tunnel of subgroups described in Definition 35. The principal graph for the subfactor given by this case (Case 15) has the following description: 
Consider the (infinite) bipartite graph $\tilde{\mathcal{G}}$ with the sets $\tilde{\mathcal{G}}^{(0)}$ (resp., $\tilde{\mathcal{G}}^{(1)}$ ) of even (resp., odd) vertices being indexed by $\coprod_{k=0}^{\infty} \hat{H}_{k}$ (where $\hat{H}$ denotes the 'unitary dual' of the (discrete) group $H$ ), and with the even vertex $(\sigma, l)_{0}$ (i.e., $\sigma \in \hat{H}_{l}$ ) connected only to odd vertices of the form $(\pi, l \pm 1)_{1}$, the number of bonds joining $(\sigma, l)_{0}$ to $(\pi, l-1)_{1}$ (resp., $\left.(\tilde{\pi}, l+1)_{1}\right)$ being given by the multiplicity with which the irreducible representation $\sigma$ of $H_{l}$ (resp., $\tilde{\pi}$ of $H_{l+1}$ ) features in the restriction to $H_{l}$ (resp., $H_{l+1}$ ) of the representation $\pi$ of $H_{l-1}$ (resp., $\sigma$ of $H_{l}$ ).

Let $\mathcal{G}$ be the connected component in $\tilde{\mathcal{G}}$ of $(1,0)_{0}$, where $(1,0)_{0}$ denotes the trivial representation of $H_{0}$ (viewed as an even vertex of $\tilde{\mathcal{G}}$ ). Then $\mathcal{G}$ is the desired principal graph.

Proof. The proof of the theorem follows from the foregoing analysis of this case, coupled with the general Proposition 10, and the verification that the identifications we have made are compatible with the various natural inclusions.

Without going through the details of the analysis, we just draw the subgraph of the principal graph induced by the set of vertices at distance at most 5 from $*$ (which is $(1,0)_{0}$ in the notation of Theorem 37).

In this case, also, we believe the principal graph is actually a tree, but do not as yet have a proof of that. We do, however, make the obvious remark that the graph is certainly infinite. This is clear from Theorem 37, and can also be deduced from Lemma 32 and Lemma 33 in exactly the same manner in which infinite depth was proved in Case (11).

\section{CONCluding REMARKS}

(a) It is true, in general, that if $u=\left(\left(u_{\beta b}^{\alpha a}\right)\right)$ is a biunitary $N k \times N k$ matrix, then the transposed matrix $u^{t}$ is also biunitary and in fact there is an isomorphism of pairs $\left(R, R_{u^{t}}\right) \cong\left(R_{1}, R\right)$, where $R_{u} \subseteq R \subseteq R_{1}$ is the basic construction; thus $u^{t}$ should be thought of as the biunitary matrix that is 'dual' to $u$. In the special case when $u$ is the biunitary permutation matrix corresponding to $\pi \leftrightarrow(\rho, \lambda)$, it follows that $u^{t}$ corresponds to $\left(\phi^{-1}, \psi^{-1}\right)$. It is seen from the list in $\S 6$, and the manner in which we obtained the reduction to the 18 special cases in $\S 6$, that each of the biunitary matrices coming from the list is equivalent to its dual; thus, if $u$ is any biunitary permutation matrix in $U(9)$, then the subfactor $R_{u} \subseteq R$ is self-dual - meaning that $\left(R, R_{u}\right) \cong\left(R_{1}, R\right)$, where $R_{u} \subseteq R \subseteq R_{1}$ is the basic construction. The following question is natural: Is it true that for any biunitary permutation matrix $u \in U(N k)$, the associated subfactor $R_{u} \subseteq R$ is always self-dual? (More generally, the authors do not know of a subfactor arising from a vertex model - as in $\S 2$ - which is not self-dual, and would like to see an example of this phenomenon.)

(b) This remark concerns what we might call the Cayley graph of a group or a group-dual corresponding to a subset $\Gamma$. ( See [P1].) Suppose $G$ (resp., $K$ ) is a discrete (resp., compact) group, and suppose $\Gamma$ is a (finite, in all cases of interest to us) subset of $G$ (resp., $\hat{K}$, the 'unitary dual' of $K$ ), possibly with repetitions. The Cayley graph of $G$ (resp., $\hat{K}$ ) with respect to $\Gamma$ is the bipartite graph $\mathcal{G}$, which we shall denote by $C(G, \Gamma)$ (resp., $C(\hat{K}, \Gamma)$ ), described as follows: let $\tilde{\mathcal{G}}$ be the bipartite graph whose sets of even and odd vertices, denoted by $\tilde{\mathcal{G}}^{(0)}$ and $\tilde{\mathcal{G}}^{(1)}$, are given by $\tilde{\mathcal{G}}^{(i)}=G \times\{i\}$ ( resp., $\hat{K} \times\{i\}$ ), for $i=1,2$. Adjacency in $\tilde{\mathcal{G}}$ is defined by the prescription that if $\left(g_{i}, i\right) \in \tilde{\mathcal{G}}^{(i)}$ (resp., $\left.\left(\pi_{i}, i\right) \in \tilde{\mathcal{G}}^{(i)}\right)$, then the number of bonds joining $\left(g_{0}, 0\right)$ to $\left(g_{1}, 1\right)$ (resp., $\left(\pi_{0}, 0\right)$ to $\left.\left(\pi_{1}, 1\right)\right)$ is given by 


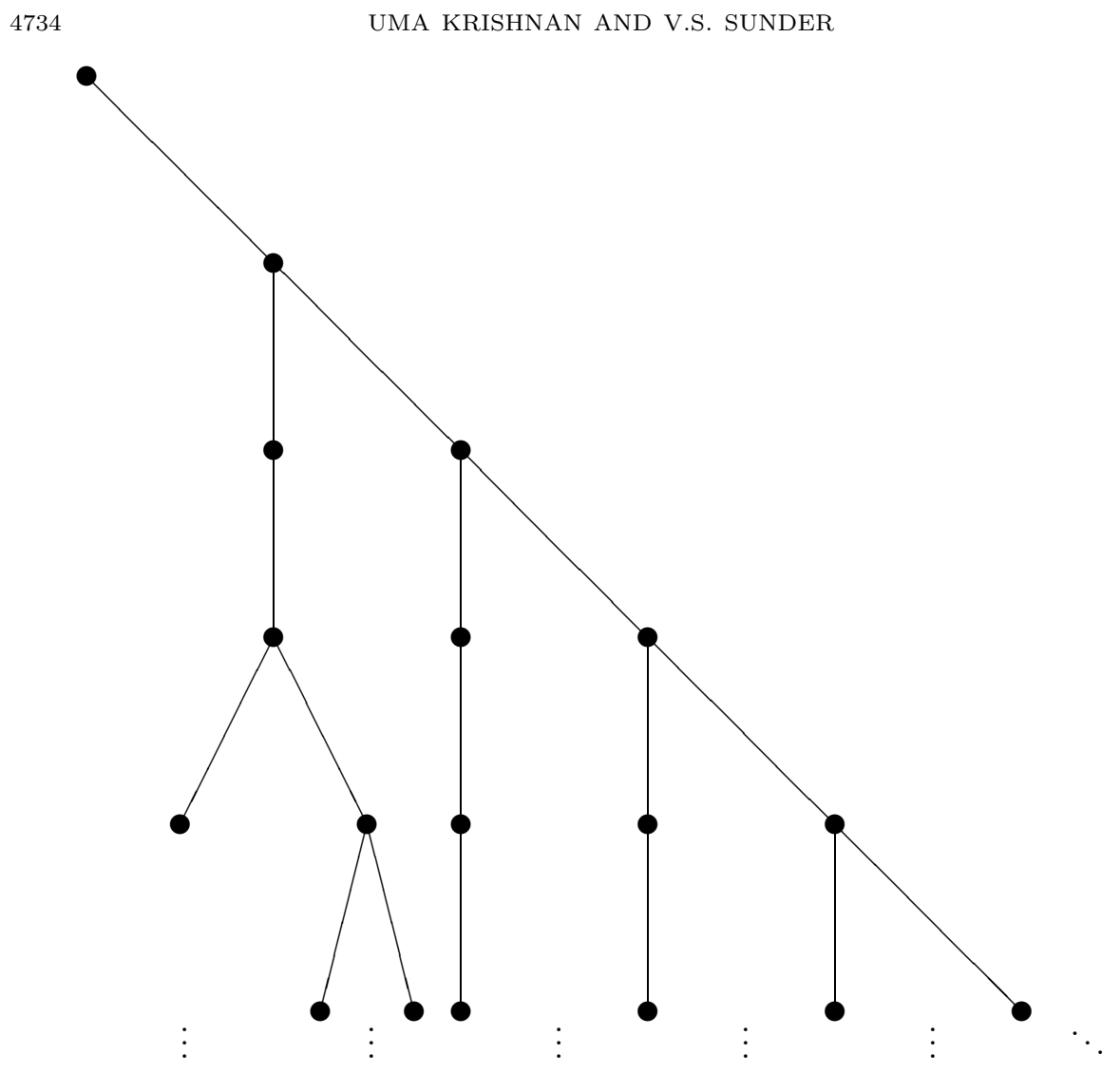

FiguRE 15

$\#\left\{\gamma \in \Gamma: g_{1}=g_{0} \gamma\right\}$ (resp., $\left.\sum_{\rho \in \Gamma}\left\langle\pi_{0} \otimes \rho, \pi_{1}\right\rangle\right)$. The Cayley graph $C(G, \Gamma)$ (resp., $C(\hat{K}, \Gamma))$ is the connected component in $\tilde{\mathcal{G}}$ of $(1,0)$, where 1 denotes the identity element of $G$ (resp., the trivial representation of $K$ ).

These were precisely the sort of graphs encountered in $\S 5$, Cases (1) and (2). By a somewhat peculiar coincidence, each of the 15 finite principal graphs encountered, when $N=3$, is of the above sort. We list below the group/group-dual and the set $\Gamma$ for each of the 15 cases in the following tabular form, for reasons that will become transparent when we make the next remark.

We do not, as yet, know of an example of a finite principal graph for a subfactor arising from a permutation biunitary matrix ( vertex model) which is not the Cayley graph of a group or a group-dual. The reason for our including Case (3) in $\S 5$ was to indicate that surely such examples must exist.

In the process of our finding the group for which a certain graph was a Cayley graph, we made a fairly simple observation which might be of interest to specialists: in order that a finite principal graph be a Cayley graph of a group, it is necessary and sufficient that the principal graph be regular - meaning that all its vertices have the same degree. 


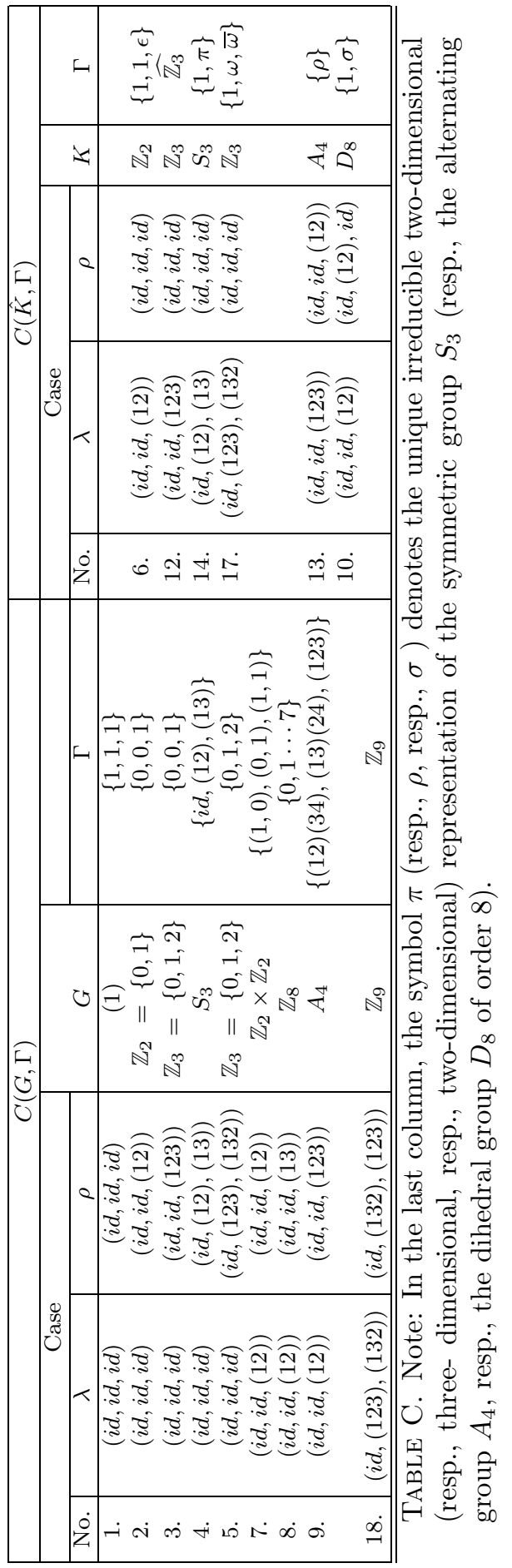


(c) This remark concerns the possible relation between the subfactors constructed out of a pair of biunitary matrices which are adjoints of one another. For biunitary permutation matrices, this means the subfactors arising from $(\rho, \lambda)$ and $\left(\psi^{-1}, \phi^{-1}\right)$. For the purposes of this remark, it will be convenient to call the biunitary permutation $\left(\psi^{-1}, \phi^{-1}\right)$ the transpose of the biunitary permutation $(\rho, \lambda)$.

The reason for Table $\mathrm{C}$ is the following: two permutation biunitary matrices which are equivalent to transposes of one another (and occur in our list) and which yield finite principal graphs are listed in the same line of the table. A line in the table consists of a single case only when that $(\rho, \lambda)$ is such that $(\rho, \lambda) \sim\left(\psi^{-1}, \phi^{-1}\right)$ in the sense of $\S 6$. Note that, whenever a line in the table has two cases listed in it, the principal graphs in those two cases are of the form $C\left(G, \Gamma_{1}\right)$ and $C\left(\hat{G}, \Gamma_{2}\right)$ for some finite group $G$ and some sets $\Gamma_{1}, \Gamma_{2}$.

It is also worth noting that the two infinite principal graphs discussed in $\S 8$ arise from a pair of biunitary permutation matrices which are transposes of one another. These observations prompt the following question.

Question: Suppose $u \in M_{N}(\mathbb{C}) \otimes M_{k}(\mathbb{C})$ is a biunitary matrix. Let $N$ (resp., $N^{*}$ ) be the subfactor of the hyperfinite factor constructed from $u$ (resp., $u^{*}$ ).

Does $N$ have finite depth precisely when $N^{*}$ does ?

Note that $N^{*}$ does not inherit irreducibility from $N$. (This is clear from the two infinite depth examples, as also from the Cayley graph examples.)

\section{REFERENCES}

[BHJ] Roland Bacher, Pierre de la Harpe and V.F.R. Jones, Carres commutatifs et invariants de structures combinatoires, C. R. Acad. Sci. Paris Sér. I. Math. 1 (1995), 1049-1054. CMP 1995:13

[GHJ] F. Goodman, P. de la Harpe and V.F.R. Jones, Coxeter graphs and towers of algebras, MSRI Publ., 14, Springer, New York, 1989. MR 91c: 46082

[HS] U. Haagerup and J. Schou, Some new subfactors of the hyperfinite $I I_{1}$ factor, preprint.

$[\mathrm{H}] \quad$ Pierre de la Harpe, Commuting squares and state models for link diagrams, Lecture given at a Conference on Subfactors at Eugene, Oregon in August 1993.

[J] V.F.R. Jones, Index for subfactors, Invent. Math. 71 (1983), 1-25. MR 84d:46097

[J1] V.F.R. Jones, Oral Communication.

[JS] V.F.R. Jones and V.S. Sunder, Introduction to subfactors, preprint.

[K] Y. Kawahigashi, On flatness of Ocneanu's connection on the Dynkin diagrams and classification of subfactors, J. Funct. Anal 127 (1995), 63-107. MR 95j:46075

[KSV] Uma Krishnan, V.S. Sunder and Cherian Varughese, On some subfactors of integer index arising from vertex models, preprint.

[O] A. Ocneanu, Quantized groups, string algebras and Galois theory for algebras, Operator Algebras and Appl., Vol. 2 (Warwick 1987), London Math. Soc. Lecture Notes Ser. Vol. 136, Cambridge University Press, 1988, pp. 119-172. MR 91k:46068

[OK] A. Ocneanu ( Lecture Notes written by Y. Kawahigashi ), Quantum Symmetry, Differential Geometry of Finite Graphs, and Classification of Subfactors, Univ. of Tokyo Seminary Notes, 1990.

[P] S. Popa, Classification of subfactors : the reduction to commuting squares, Invent. Math. 101 (1990), 19-43. MR 91h:46109

[P1] S. Popa, Sur la classification des sous-facteurs d'indice fini du facteur hyperfini, C. R. Acad. Sci. Paris Ser. I 311 (1990), 95-100. MR 91m:46094

[P2] S. Popa, Classification of amenable subfactors of type II, Acta Math. 172 (1994), 163-255. MR 95f: 46105

Stat-Math Unit, Indian Statistical Institute, R.V. College Post, Bangalore-560059, INDIA 